
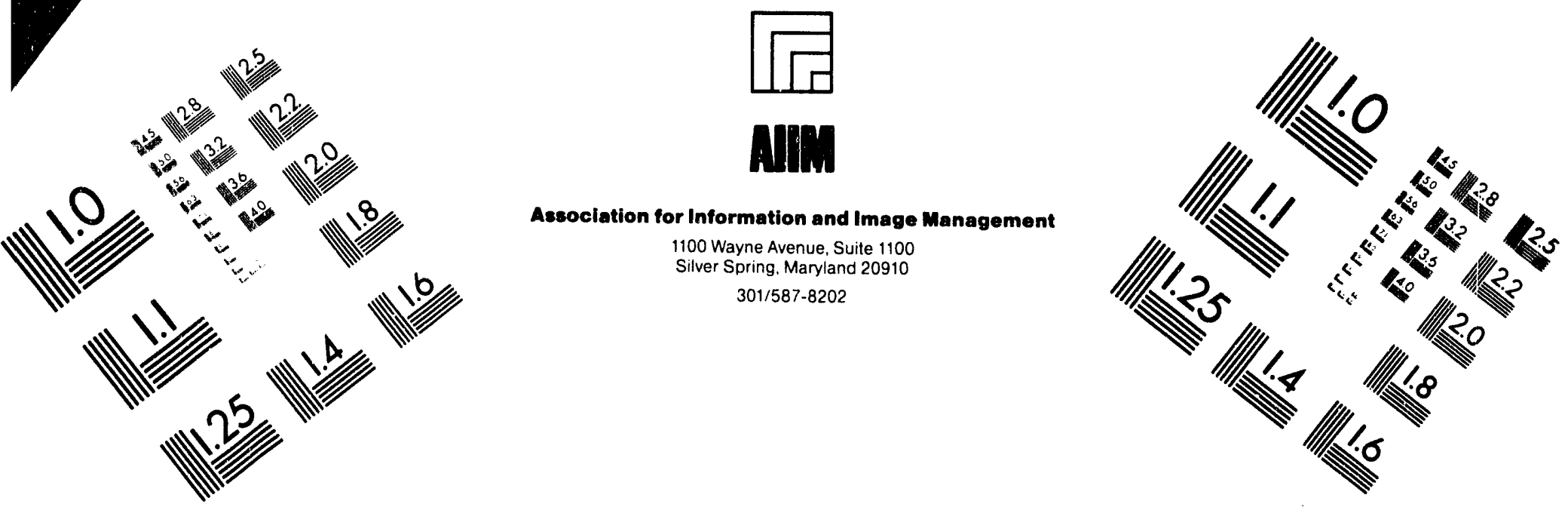

Centimeter

1m Inches
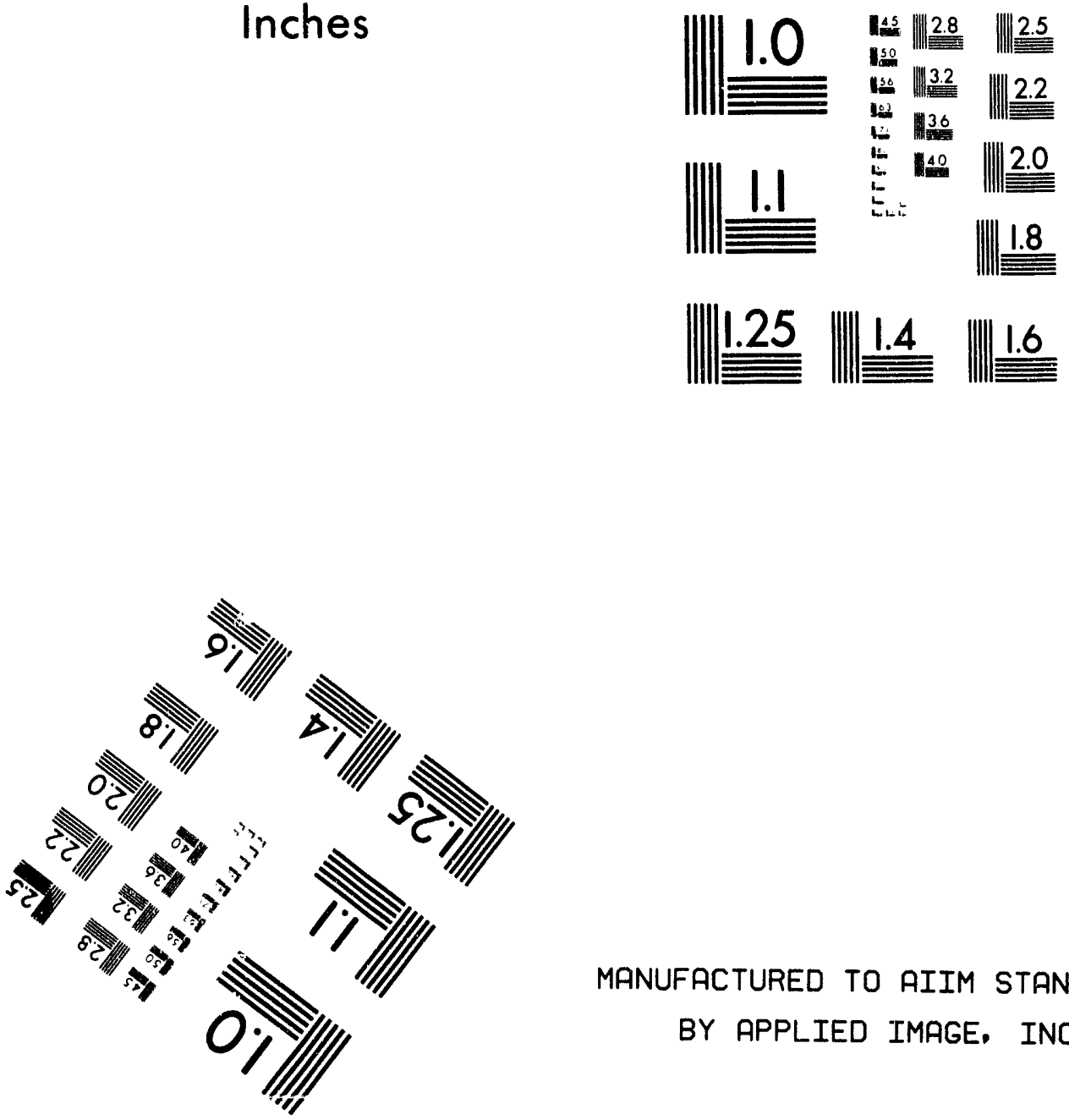

MANUFACTURED TO AIIM STANDARDS

BY APPLIED IMAGE, INC.

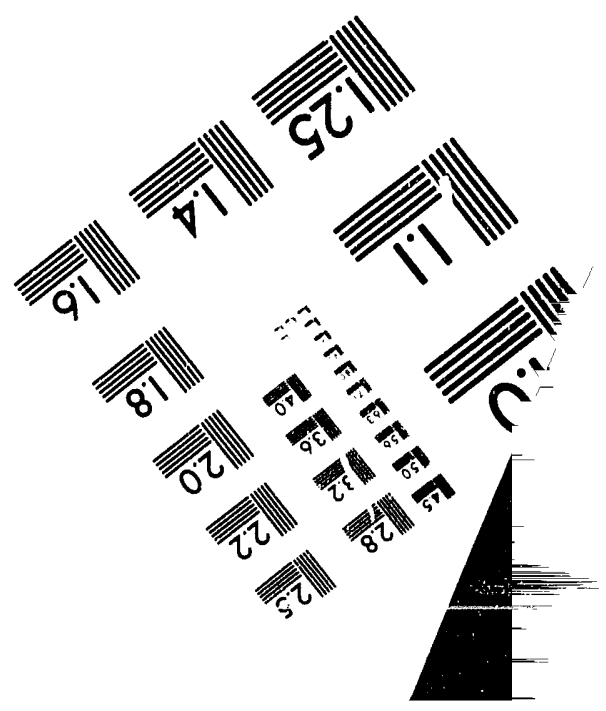



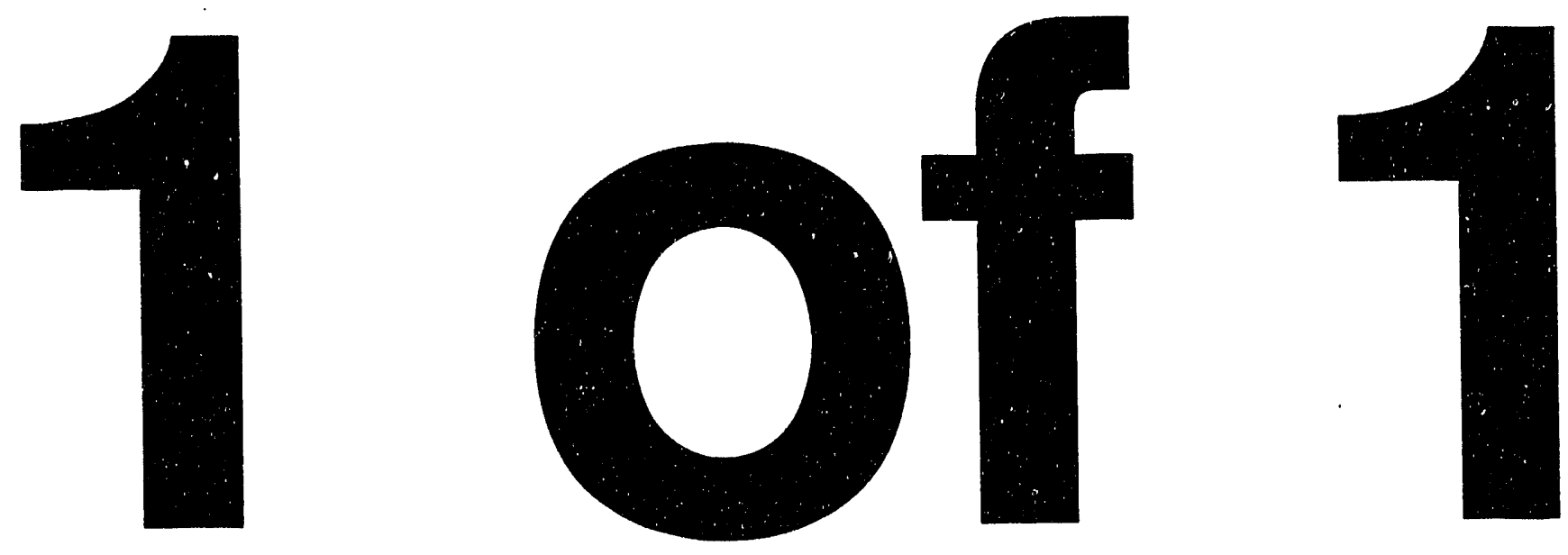


\section{Hydrogen Mixing Studies (HMS) Assessment Manual}

Manuscript Completed: May 1993

Date Published: June 1993

Prepared by

K. L. Lam, T. L. Wilson, J. R. Travis*

Los Alamos National Laboratory

Los Alamos, NM 87545

\section{DISCLAIMER}

Prepared for

Division of Systems Research

Office of Nuclear Regulatory Research U.S. Nuclear Regulatory Commission Washington, DC 20555-0001

NRC FIN A 7247
This report was prepared as an account of work sponsored by an agency of the United States Government. Neither the United States Government nor any agency thereof, nor any of their employees, makes any warranty, express or implied, or assumes any legal liability or responsibility for the accuracy, completeness, or usefulness of any information, apparatus, product, or process disclosed, or represents that its use would not infringe privately owned rights. Reference herein to any specific commercial product, process, or service by trade name, trademark, manufacturer, or otherwise does not necessarily constitute or imply its endorsement, recommendation, or favoring by the United States Government or any agency thereof. The views and opinions of authors expressed herein do not necessarily state or reflect those of the United States Government or any agency thereof.
* Presently with

Science Applications International Corporation 2109 Air Park Rd., SE

Albuquerque, NM 87106

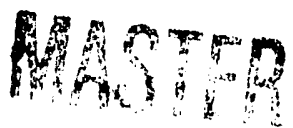

DISTRIEUTION OF THIS DOCUMENT IS UNLIMITED 


\title{
HYDROGEN MIXING STUDIES (HMS): ASSESSMENT MANUAL
}

\author{
by \\ K. L. Lam, J. R. Travis, and T. L. Wilson
}

\begin{abstract}
This report documents some calculations performed tc assess the Hydrogen Mixing Studies (HMS) code. Results are presented first for some analytical test problems, including laminar flow and mass diffusion. Then we discuss the von Karman vortex street problem, the Sandia FLAME Facility test problem, and the Heiss Dampf Reaktor (HDR) containment facility test problem. For the analytical problems, the code gave results that agree exceptionally well with the analytical solutions.

Calculations for the von Karman vortex street problem were performed at selected Reynolds numbers for several obstacle types. The computed flow patterns agree well with experimental observationsspecifically the occurrence of a vortex street (double row of vortices) above a critical Reynolds number. The calculated Strouhal number or nondimensional vortex-shedding frequency agrees well with the empirical values.

The Sandia FLAME Facility test problem involved injection of hydrogen horizontally and near the bottom into a long char nel $(1.83 \mathrm{~m}$ wide, $2.44 \mathrm{~m}$ high, and $30.5 \mathrm{~m}$ long) that had been initially filled with air. Because operating fans were not used to mix the gases, a vertical s,tratification of the hydrogen occurred. The calculations show the develcpment of a buoyant plume and the same level of hydrogen stratification that was observed in the experiment $30 \mathrm{~min}$ after the injection.

The last assessment problem involves modeling the experiment T31.5. The experiment was carried out in the HDR containment building, which is a large, multicompartment facility ( $11300 \mathrm{~m}^{3}$ free volume in 72 compartments). In the experiment, a steam-water mixture was first injected into the containment to simulate a large-break blowdown of a pressure vessel, and then superheated steam was injected that was followed by a release of helium-hydrogen light gas. The calculated results (pressure, temperature, and gas concentrations) agree reasonably well with the experimental data.
\end{abstract}




\section{SUMMARY AND CONCLUSIONS}

The Los Alamos National Laboratory is developing the Hydrogen Mixing Studies (HMS) code for the U. S. Nuclear Regulatory Commission as a best-estimate tool to predict the transport, mixing, and combustion of hydrogen and other gases in nuclear reactor containments and other facilities. To validate the methodology and models used in the code, we used both analytical and experimental test problems to asse $s$ the code. The tests included transient laminar flow and hydrogen mass diffusion theoretical problems, the classical von Karman vortex street problem, a Sandia FLAME Facility experiment, and the T31.5 blowdown and hydrogen distribution experiment performed at the large-scale German HDR Containment Facility $\left(11000 \mathrm{~m}^{3}\right.$ free volume in 72 interconnected compartments).

The excellent agreement between theoretical and HMS calculated results of the transient laminar flow and mass diffusion test problems prove that the numerical procedure used for solving these problems is correct and sound. Good results were also obtained for the classical von Karman vortex street problem, which does not yield to analytical solutions but can be compared to some experimental data. In agreement with experimental data, the code predicts the occurrence of vortex street patterns above (and none below) a critical Reynolds number and reproduces reasonably well the vortexshedding frequencies for flow obstacles of various shapes. These are indications that the code's basic fluid-dynamics calculations are robust and accurate. Therefore, we are confident that the code provides a sound Navier-Stokes solver on which additional constitutive models (such as heat transfer, turbulence, and combustion) can be built.

The Sandia FLAME problem involves development of a buoyant plume from a small but strong hydrogen jet in a channel $\left(136 \mathrm{~m}^{3}\right)$ that was initially filled with air at ambient conditions. Thirty minutes after the hydrogen injection was terminated, the experiment showed that the hydrogen distribution was still stratified because mixing fans were not used. The calculation predicts to within measurement uncertainties that the amount of hydrogen at the top and at the bottom of the FLAME facility is 15\% and $10 \%$ mole fraction, respectively.

For the HDR containment test problem, the calculation confirms the suitability of an isentropic expansion model to determine the correct containment steam source from a high-pressure steam-water blowdown jet. The HMS calculated containment pressure, especially its peak value, agrees very well with experimental data. Calculated temperatures at three locations show fairly good agreement with experimental data. In general, the temperature was overpredicted at some locations, which was interpreted to 
be due to the assumption in the calculation that the initial temperature in the containment was uniformly at $32^{\circ} \mathrm{C}$, which was not true during the experiment. As a result, at some locations the HMS calculations assumed a temperature that is about $10^{\circ} \mathrm{C}$ higher than that in the exp -iment. The calculated hydrogen distribution reproduces the data fairly well, although the level of stratification is slightly too high when compared with the measurement. Overall, the accuracy in this calculation, which involves multiple gas species, heat transfer, and condensation in an extremely complicated geometry, is commensurate with the level of detail of the computational model and with the simplifying assumptions (such as constant transport properties and uniform initial temperature distribution) used in specifying the problem. 


\section{CONTENTS}

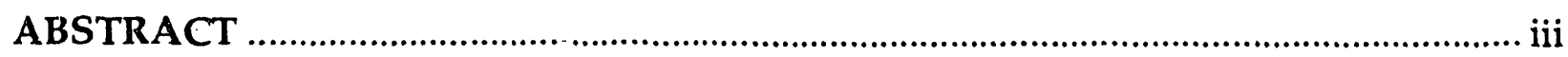

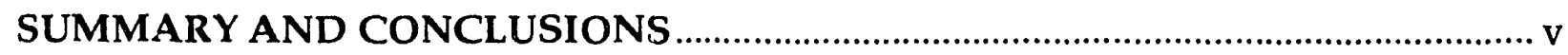

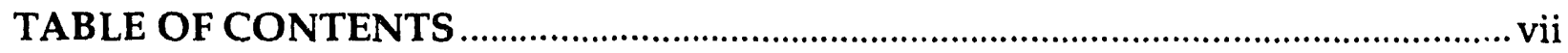

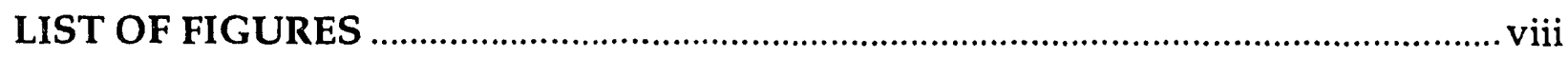

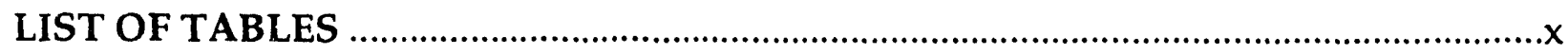

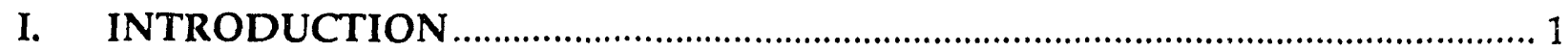

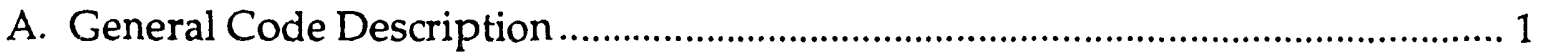

B. Assessment Problems ...................................................................................... 2

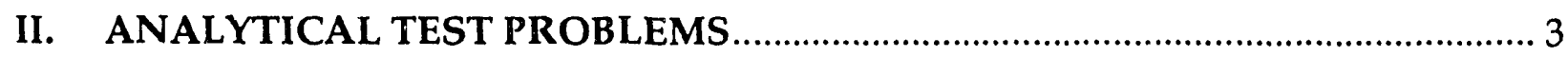

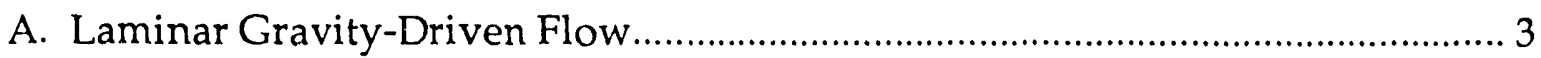

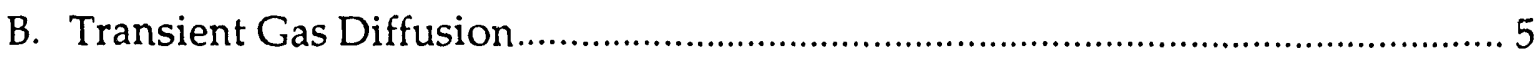

III. VON KARMAN VORTEX STREET PROBLEM ……........................................... 7

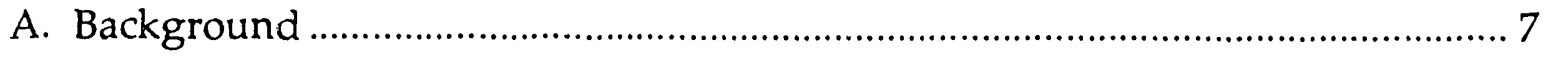

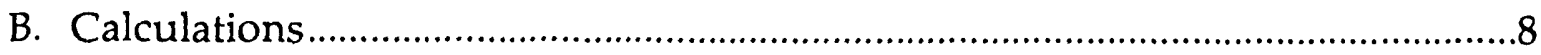

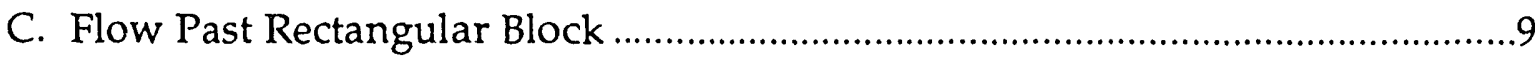

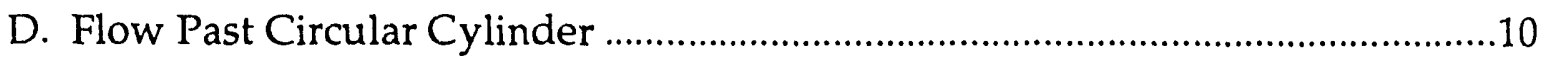

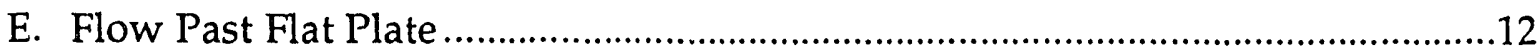

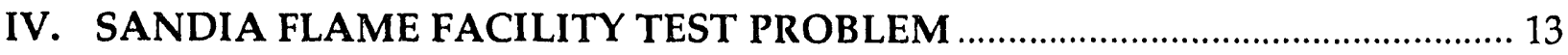

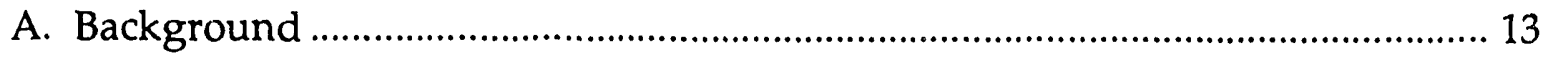

B. HMS Model of FLAME Facility ..................................................................... 14

C. Calculation Results and Comparison with Experiment ................................... 15

V. HDR CONTAINMENT FACILITY TEST PROBLEM ….......................................18

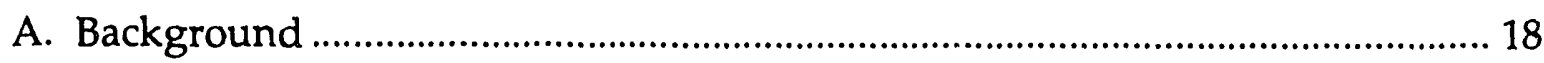

B. HMS Model of HDR Containment Facility …….............................................. 19

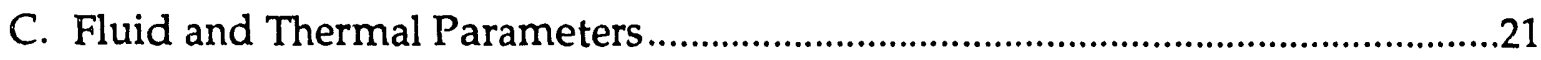

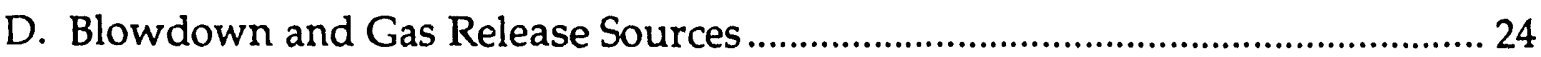

E. Calculation Results and Comparison with Experiment ................................... 27

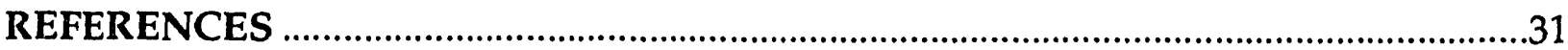




\section{LIST OF FIGURES}

FIGURE 1 Computed velocity profiles at various times.............................................33

FIGURE 2 Logarithm of $u^{\prime}$ at $y=4 \mathrm{~cm}$ for the laminar test problem.........................34

FIGURE 3 Computed profiles of hydrogen mole fraction at two time instants compared to the analytical solution for the mass diffusion test problem

FIGURE 4 Computational mesh (variable grid) used for the flow past rectangular block problem. 36

FIGURE 5 Velocity vector plot for flow around a rectangular block 37

FIGURE 6 Time-history plots of the normal and streamwise velocity components at two locations for flow around a rectangular block... 38

FIGURE 7 Computational mesh (uniform) for the flow past circular cylinder problem 39

FIGURE 8 Velocity-vector plot for flow around a cylinder, $\operatorname{Re}=131$ at $t=10 \mathrm{~s}$. 40

FIGURE 9 Time-history plots of the normal and streamwise velocity components for flow over a cylinder, $\operatorname{Re}=131$

FIGURE 10 Time-history plots of the normal and streamwise velocity components for flow over a cylinder, $\mathrm{Re}=131$

FIGURE 11 Velocity-vecior plot for flow around a cylinder, $\operatorname{Re}=1000$ at $t=1 \mathrm{~s}$..

FIGURE 12 Time-history plots of the normal and streamwise velocity components for flow over a cylinder, $\operatorname{Re}=1000$

FIGURE 13 Velocity-vector plot for flow around a cylinder, $\operatorname{Re}=\mathbf{1 0 0 0 0}$ at $\mathrm{t}=6 \mathrm{~s}$........ 45

FIGURE 14 Time-history plots of the normal and streamwise velocity components for flow over a cylinder, $\operatorname{Re}=\mathbf{1 0 0 0 0}$

FIGURE 15 Schematic diagram of the Sandia FLAME channel showing locations of hydrogen injectirn.

FIGURE 16 Computational domain for the Sandia FLAME simulation calculation showing the variable mesh 48

FIGURE 17 Two dimensional view of the computational mesh used for the Sandia FLAME calculations

FIGURE 18 Plot of the calculated velocity vectors in the Sandia FLAME experiment 50

FIGURE 19 Contour plot of the calculated hydrogen volume fraction in the Sandia FLAME experiment, at 100s 
FIGURE 20 Contour plot of the calculated hydrogen volume fraction in the Sandia FLAME experiment, at 200s 52

FIGURE 21 Plot of the calculated velocity vectors in the Sandia FLAME experiment, at $487 \mathrm{~s}$

FIGURE 22 Computed hydrogen volume fraction contours in the Sandia FLAME experiment, at $487 \mathrm{~s}$ 54

FIGURE 23 Calculated profile of hydrogen mole fraction in the Sandia FLAME experiment. .55

FIGURE 24 Calculated contours of hydrogen mole fraction at three vertical planes at $117 \mathrm{~s}$ after the injection stops. 56

FIGURE 25 Calculated hydrogen volume fraction contours in the Sandia FLAME experiment at the plane of injection $1800 \mathrm{~s}$

FIGURE 26 Time-history plots of the calculated hydrogen mole fraction in the Sandia FLAME experiment at various locations 58

FIGURE 27 Cross-Sectional view of the HDR cortainment building at two angles

FIGURE 28 Cross-Sectional view of the HDR containment facility showing the blowdown room

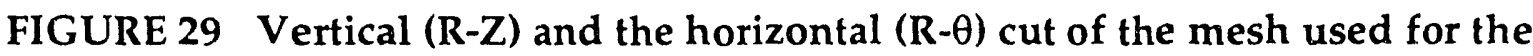
HDR calculation

FIGURE 30 Horizontal cross-section of the HDR containment facility at $16 \mathrm{~m}$

FIGURE 31 HDR vertical cross section at $10^{\circ}$

FIGURE 32 Temperature-Entropy phase diagram (schematic) for water showing the isentropic expansion process from the blowdown jet pressure $\left(P_{\text {con }}\right)$.

FIGURE 33 Blowdown mass flow rates for two time intervals as given by the source data.

FIGURE 34 Pressure in the blowdown jet (source data) and in the containment (calculated by HMS) for two time intervals

FIGURE 35 Temperature in the blowdown jet (source data) and in the containment source (calculated) after the isentropic expansion process for two time intervals.

FIGURE 36 Steam qualities in the blowdown jet and in the containment source after the isentropic expansion for two time intervals. 68

FIGURE 37 Calculated steam and liquid source rates into the containment after the isentropic expansion process for two time intervals 
FIGURE 38 Steam masses in original blowdown jet (source data) and in source to containment (calculated) for two time intervals

FIGURE 39 Liquid masses in original blowdown jet (source data) and in expanded source to containment (calculated) for two time intervals ..... 71

FIGURE 40 Total amount of steam that has been injected into the containment (by the blowdown) compared to the actual amount of steam in the containment for two time intervals

FIGURE 41 Conditions of superheated steam and light gas releases from $21 \mathrm{~min}$ to $50 \mathrm{~min}$ after beginning of the blowdown as given by the source data. 73

FIGURE 42 Calculated cumulative steam source (after expansion from pipe to containment) and the actual steam mass (also calculated) in the containment during the first hour. 74

FIGURE 43 Calculated velocity vector plots at three vertical planes shortly $(7 \mathrm{~ms})$ after initiation of the large-break blowdown ............................................ 75

FIGURE 44 Calculated velocity vector plots at $22 \mathrm{~min}$ at three vertical planes...........76

FIGURE 45 Calculated velocity vector plots at $40 \mathrm{~min}$ at three vertical planes:..........77

FIGURE 46 Comparison between the HMS-calculated pressure and experimental measurement in the HDR T31.5 Test.................................78

FIGURE 47 Comparison between the HMS-calculated temperature and experimental measurement at three locations in the HDR T31.5 Test....79

FIGURE 48 Comparison between the HMS-calculated hydrogen concentration and experimental measurement in the HDR T31.5 Test.

FIGURE 49 Measured and HMS-calculated concentrations of air, steam, and hydrogen at the top of the dome in the HDR T31.5 Test

\section{LIST OF TABLES}

TABLE 1 Some parameters for the von Karman vortex street computational runs.

TABLE 2 Location of the X-, Y-, and Z-grid lines in the HMS model of the Sandia FLAME facility

TABLE 3 Time sequence of events in the HDR T31.5 experiment

TABLE 4 Discretization of the 3D computational volume for HDR containment facility

TABLE 5 Specification of internal heat sinks for HDR containment model 


\section{INTRODUCTION}

\section{A. General Code Description}

The Los Alamos National Laboratory is developing the Hydrogen Mixing Studies (HMS) code for the U. S. Nuclear Regulatory Commission as a best-estimate tool for predicting the transport, mixing, and combustion of hydrogen and other gases in nuclear reactor containments and other facilities. The code can model geometrically complex facilities having multiple compartments and internal structures. It can simulate the effects of condensation, heat transfer to walls and internal structures, chemical kinetics, and fluid turbulence.

HMS is a finite-volume code based on proven computational fluid dynamics methodology that solves the three-dimensional (3D), compressible Navier-Stokes equations. Transport equations for the internal energy and for multiple gas species are also solved. The computational domain is discretized by a mesh of regular orthogonal cells in either parallelepiped Cartesian or right cylindrical geometry, where the primary hydrodynamic variables are cell-face-centered normal velocity and cell-volume-centered density, internal energy, and pressure. A linearized Arbitrary-Lagrangian-Eulerian method is used for approximating the solution to the coupled mass, momentum, and energy conservation equations. The implicit, iterative pressure computation in this method, which uses an efficient preconditioned conjugate gradient matrix solver, allows simulations of low-speed (low-Mach-number) flows without the time-step restrictions that are caused by the fluid sound speed.

HMS uses three turbulent models-algebraic, subgrid-scale, and $k-\varepsilon$, which are the respective zero-, one-, and two-transport equation models that compute the turbulent velocity and length scales required to determine the turbulent diffusivity. The turbulent diffusion coefficient, together with its molecular counterpart, is included in the diffusion terms in the momentum, the internal energy, and the species mass transport equations.

Heat conduction within walls and structures is one-dimensional. Heat transfer and condensation to walls and structures are calculated from the Reynolds analogy between momentum, heat, and mass transfer. A model is available to account for the enhanced mass- and heat-transfer rates in the presence of high mass fluxes toward the wall (e.g., during steam condensation). A term that accounts for the cooling effect caused by gas expanding into the volume space vacated by steam condensation is included in the energy equation. 
Chemical energy of combustion involving hydrogen or other fuels provides a source of energy within the gaseous region. HMS uses a one-step, global, chemicalkinetics model to simplify the actual chemical processes. The model is based on a modified Arrhenius rate law that calculates the local fuel and oxidizer concentrations. The finite-rate chemical equation is solved implicitly for the fuel concentration when the fuel-oxidizer mixture is fuel-lean and for the oxidizer or reactant concentration when the fuel-oxidizer mixture is fuel-rich. The procedure ensures that combustion gas components will never be driven negative, regardless of the time-step size.

A more in-depth discussion of the theory and methodology on which the HMS code is based can be found in Ref. 1 .

\section{B. Assessment Problems}

This document reports some calculations that we have performed to assess the HMS code. We begin with some test problems with known analytical solutions (Sec. II). These analytical test problems are simple to set up, do not require much computer time and memory, and therefore, are ideal for debugging after a major revision to the code or when the code is being implemented on a different machine. However, these problems check out only the gener-1 coding because rather simple geometry and physical processes are involved. The remaining assessment problems, presented in Secs. III, IV, and $\mathrm{V}$, involve more complicated geometry and physics. This set of assessment problems was chosen to test both the general methodology and various physical models of the code. In particular, the last problem, which involves modeling a large-break blowdown and hydrogen-distribution experiment, represents a typical application of the HMS code in the area of nuclear safety analysis.

The von Karman vortex street problem (Sec. III) is a classical fluid-dynamics problem that many investigators have studied, both experimentally and theoretically. A major observation reported in the studies was that a vortical wake pattern exists in flows past an obstacle when the Reynolds number is sufficiently high. This problem was chosen to test the general fluid dynamics calculations with flow obstacles and the multidimensional viscous stress calculation, in particular. The accuracy of the code will be judged by correct predictions of flow patterns (e.g., existence or absence of a vortex street when the Reynolds number is above or below the critical value) and close agreement between the calculated and experimental vortex-shedding frequencies.

In Sec. IV, we present the simulation calculations of an experiment performed at the Sandia FLAME Facility. The experiment involved injection of hydrogen horizontally and near the bottom into a rectangular channel ( $1.83 \mathrm{~m}$ wide, $2.44 \mathrm{~m}$ high, $30.5 \mathrm{~m}$ long, 
with a total volume of $136 \mathrm{~m}^{3}$ ) that $j$ initially was filled with air. Because operating fans were not used to mix the gases, a buoyant plume developed that caused vertical stratification of the hydrogen concentration. This problem tests the mass diffusion model and, to some extent, the algebraic turbulence model. No extensive measurements on hydrogen concentration were made in the experiment, except at two locations (one at the top and the other at the bottom of the facility) $30 \mathrm{~min}$ after the injection had stopped. The calculated hydrogen concentrations are compared with the measured values at these locations.

The ?ast assessment calculation, reported in Sec. V, involves modeling the Heiss Dampf Reaktor (HDR) containment building, which is a large facility with 72 compartments interconnected by about 200 passageways and a large number of steel structures. The net free volume of the containment is $11300 \mathrm{~m}^{3}$. The experiment being simulated is T31.5. A steam-water mixture was injected into the containment at a high flow rate for about $1 \mathrm{~min}$ and after $20 \mathrm{~min}$, superheated steam was injected for $15 \mathrm{~min}$. A light-gas ( $85 \%$ helium and $15 \%$ hydrogen by volume) release followed immediately and continued for another $12 \mathrm{~min}$. Available data include temperature, pressure, and gas concentrations at various locations in the containment. This assessment problem involves multicomponent fluid flow in a multicompartment, cylindrical geometry and the integral effects of many physical processes (e. g., heat transfer, condensation, buoyancy) on the fluid-flow behavior. This problem also tests a blowdown model that expands the two-phase, steam-water mixture from the blowdown pipe to the containment and calculates the appropriate steam source received by the containment.

\section{ANALYTICAL TEST PROBLEMS}

\section{A. Laminar Gravity-Driven Flow}

Definition of the Analytical Problem. The flow of a Newtonian fluid between two parallel stationary walls is the first problem. The flow is driven by a constant body force caused by gravity. For flows that are two-dimensional, laminar, and incompressible, the following simplified Navier-Stokes equation applies:

$$
\frac{\partial u}{\partial t}=v \frac{\partial^{2} u}{\partial y^{2}}+g \quad\left\{\begin{array}{l}
u(y, t=0)=0 \\
u(y=0, t>0)=u(y=H, t>0)=0 .
\end{array}\right.
$$


In the equations above, $u$ is the velocity in the direction of the gravitational force $g, v$ is the kinematic viscosity of the fluid, $t$ is the time coordinate, and $y$ is the spatial coordinate extending from 0 at one wall to $H$ at the other wall. Initially the fluid is at rest, and because of the no-slip boundary condition, $u=0$ at both walls. The method of separation of variables can be used to solve this problem analytically. The solution can be expressed as

$$
u(y, t)=u_{s s}(y)+\sum_{n=1,2}^{\infty} c_{n} e^{-\left(\frac{n \pi}{H}\right)^{2} v t} \sin \frac{n \pi}{H} y,
$$

where

$$
c_{n}=\frac{2}{H} \int_{0}^{H}-u_{s s}\left(y^{\prime}\right) \sin \frac{n \pi}{H} y^{\prime} d y^{\prime},
$$

and the steady-state solution $u_{s s}(y)$ is given by

$$
u_{s s}(y)=\frac{g y}{2 v}(H-y) .
$$

It can be seen from the analytical solution that the transient behavior of the velocity is governed by a summation series of negative exponential functions with decay constants

$$
\lambda_{n} \equiv v\left(\frac{n \pi}{H}\right)^{2} \text {. }
$$

Computational Run. The test problem simulates laminar flow of air at a Reynolds number (based on the average velocity and channel height) of 100 . The values for some of the parameters are

$$
H=8 \mathrm{~cm}, \quad v=0.153 \mathrm{~cm}^{2} / \mathrm{s}, \quad g=0.054865 \mathrm{~cm} / \mathrm{s}^{2} .
$$

The steady-state maximum and average velocities for this problem are

$$
u_{\max }=2.8688 \mathrm{~cm} / \mathrm{s}, \quad u_{\mathrm{avg}}=1.9125 \mathrm{~cm} / \mathrm{s}
$$


For the calculations, the computational domain size is $8 \mathrm{~cm}$, divided into 33 cells, in the $y$-direction and $4 \mathrm{~cm}$, represented by one cell, in the $x$-direction. The time-step size was $\Delta t=0.1 \mathrm{~s}$. We confirmed that this choice of the discretization parameters satisfies both the Courant and diffusion-limit criteria for numerical stability. The computation was carried out for a problem time of $200 \mathrm{~s}$.

Results. A plot of the computed velocity profiles in the channel at various times is presented in Fig. 1. Also plotted is the analytical solution at steady state. It can be seen that the velocity profile, as expected, approaches the steady parabolic distribution asymptotically as time increases. The first or dominant decay constant of the exact solution is

$$
\lambda_{1}=v\left(\frac{\pi}{H}\right)^{2}=0.0236 \mathrm{~s}^{-1}
$$

The othei decay conotants are much larger $\left(\lambda_{2}=0.044 \mathrm{~s}^{-1}, \lambda_{3}=0.212 \mathrm{~s}^{-1}\right.$, etc. $)$ and the corresponding time exponentials will die oi: aits roughly $\tau_{2} \equiv 1 / \lambda_{2} \approx 10 \mathrm{~s}$. The decay constant for the computed solution can be obtained from the transient behavior of $u^{\prime}(y, t) \equiv u(y, t)-u_{s s}(y)$ at a point. Figure 2 is a plot of the natural logarithm of $u^{\prime}$ at the centerline or $y=4 \mathrm{~cm}$. The plot appears largely linear, indicating that $u^{\prime}$ decays exponentially in time. The slope, as calculated from two points at $t=40 \mathrm{~s}$ and $t=140 \mathrm{~s}$, is $-0.0236 \mathrm{~s}^{-1}$. This value agrees with the theoretical decay time constant $\lambda_{1}$ to three significant figures.

\section{B. Transient Gas Diffusion}

Definition of the Analytical Problem. Consider the transient diffusion of gas A in a semi-infinite slab of gas B. If Fick's law applies to the binary diffusion process with a constant diffusivity relating the mass flux of gas A to the gradient in its mole fraction, then

$$
\frac{\partial f}{\partial t}=D_{A B} \frac{\partial^{2} f}{\partial x^{2}} \quad\left\{\begin{array}{l}
f(x, t=0)=f_{o} \\
f(x=0, t>0)=f_{b}
\end{array}\right.
$$

In the above equations, $f$ is the mole fraction of $\mathrm{A}$ in the gas mixture, $D_{A B}$ is the binary diffusion coefficient, $t$ is the time coordinate, and $x$ is the spatial coordinate extending from 0 to $\infty$. Initially the mole fraction everywhere is $f_{0}$. Then suddenly, at $x=0$, the 
mole fraction is changed from $f_{0}$ to $f_{b}$, where it is held. The well-known solution to this penetration problem is as follows:

$$
\Delta=\operatorname{erfc}(\xi),
$$

where

$$
\Delta \equiv \frac{f-f_{o}}{f_{b}-f_{o}}, \text { and } \xi \equiv \frac{x}{2 \sqrt{D_{A B} t}}
$$

It is seen from the solution that $\Delta$, a measure of the accomplished change compared with the total possible change, is given by the complementary error function of $\xi$, which is formed by combining the independent variables $x$ and $t$.

Computational Run. The next test problem chosen is the transient diffusion of hydrogen into a medium of stagnant air. Therefore, $f_{0}=0$ and $f_{b}=1$, where $f$ represents the mole fraction of hydrogen. The problem is one-dimensional, with the spatial coordinate, $x$, extending from 0 to $10 \mathrm{~cm}$. The value of the mutual diffusion coefficient, $D_{A B}$, in a hydrogen-air mixture is estimated from the kinetic theory of gases to be $0.754 \mathrm{~cm}^{2} / \mathrm{s}$.

For the calculations, the computational domain of $10 \mathrm{~cm}$ is divided into 100 cells, so $\Delta x$ is $0.1 \mathrm{~cm}$. The time-step size was $\Delta t=0.005 \mathrm{~s}$. The choice of $\Delta x$ and $\Delta t$ satisfies the diffusion-limit criterion for numerical stability, which states that the diffusion number should be smaller than one half, or

$$
\frac{D_{A B} \Delta t}{\Delta x^{2}}<\frac{1}{2}
$$

In the present calculation, the diffusion number is 0.377 . The computation was carried out for a problem time of $10 \mathrm{~s}$.

Results. The analytical solution of this problem shows that at any time instant, the profile of the hydrogen mole fraction is described by the complementary error function. Hence,

$$
f(x, t=5 \mathrm{~s})=\operatorname{erfc}(0.2575 x), \text { and } f(x, t=10 \mathrm{~s})=\operatorname{erfc}(0.1821 x)
$$


Figure 3a is a plot of the hydrogen mole fraction as a function of $x$ at $t=5 \mathrm{~s}$ and $t=10 \mathrm{~s}$. The corresponding theoretical profiles are shown as well. It can be seen that the computed and analytical results agree well. A second calculation was performed (shown in Fig. 3b) to ensure that the discrepancy between the computed and analytical results could be removed by improved spatial and temporal resolution. In that run, $\Delta x$ was halved $(=0.05 \mathrm{~cm})$ and $\Delta t$ accordingly adjusted to satisfy the stability requirement. The profiles of the hydrogen mole fracion (not shown), obtained at $t=5 \mathrm{~s}$ and at $t=10 \mathrm{~s}$, were then indistinguishable from the analytical predictions when plotted on the same scale used in Fig. 3a.

\section{VON KARMAN VORTEX STREET PROBLEM}

\section{A. Background}

Consider the flow of a fluid around an obstacle (e.g., a circular cylinder). In previous experimental studies, many investigators have observed that the flow pattern in the wake region undergoes several transitions as the flow speed increases. Furthermore, the flow patterns for a two-ciimensional problem are similar with respect to the Reynolds number, which is defined as

$$
\operatorname{Re} \equiv \frac{U_{\infty} d}{v},
$$

where $U_{\infty}$ is the velocity in the free stream, $d$ is the height of the obstacle (diameter in case of a cylinder), and $v$ is the kinematic viscosity of the fluid. At very low Reynolds numbers, the flow pattern is steady and laminar, with streamlines wrapping around the obstacle. As Re increases (to about 20), a pair of counter-rotating eddies appear behind the obstacle. The double eddies elongate but remain bounded as the Reynolds number is increased further until $\operatorname{Re} \approx 40$, when a dynamic wake pattern begins to appear. Eddies are alternatively shed from the top and bottom of the obstacle in a regular fashion, leaving in their wake a double row of counter-rotating vortices. The double row of vortices is called the von Karman vortex street from the pioneering work of von Karman in this area (Ref. 2). At even higher $\operatorname{Re}\left(\sim 10^{3}\right)$, the wake flow is less regular, but a distinct vortex shedding frequency is still observed. Finally, at very high $\operatorname{Re}\left(\sim 10^{6}\right)$, the flow becomes fully turbulent with an irregular wake pattern. 
Quantitatively, the vortex shedding frequency has been traditionally expressed in terms of a nondimensional number called the Strouhal number, which is defined as

$$
\mathrm{St} \equiv \frac{n d}{U_{\infty}}
$$

where $n$ is the vortex shedding frequency from each side of the obstacle. From experimental data, correlations of St as a function of Re are available for obstacle types such as the circular cylinder and flat plate at various angles of incidence.

\section{B. Calculations}

We have used HMS to calculate flows around obstacle types including the cylinder, rectangular block, and flat plate placed normal to the oncoming flow. The following results are presented only for those cases where reliable experimental data are available. An exhaustive study of the von Karman vortex street phenomenon is, of course, not the purpose of this work. Therefore, only a few flow problems selected for the assessment calculations are discussed here.

In all calculations for the von Karman vortex street problem, the flow was twodimensional in an $x-y$ Cartesian geometry. Air at normal temperature and pressure was chosen as the flowing fluid. Fluid at rest everywhere was the initial condition. The boundary conditions consisted of specified velocity at the inlet flow boundary and specified pressure at the outlet flow boundary. (At the inlet, a constant velocity was specified after an initial perturbation for a short instant of 0.1 or $1 \mathrm{~s}$, depending on the case. The initial perturbation, whose detail and magnitude is unimportant, was used to reduce the amount of time required for developing the final flow pattern.) At the boundaries representing the walls of the channel (in the experiments, the channel was usually a wind tunnel), the free-slip boundary condition was used. At the surfaces of the obstacle, the boundary condition applied was no-slip.

Some preliminary calculations showed that the computed results depended on the ratio of the channel height to the obstacle height, $H / d$, which in the literature has been called the constriction ratio. Ideally the constriction ratio should be infinite or very large to eliminate the effects of the channel walls. An $\mathrm{H} / \mathrm{d}$ of 12 was chosen for all the cases reported here. This constriction ratio was determined roughly from balancing the computational resource requirement against matching the actual experimental conditions. We performed many experiments at constriction ratios of 13 or higher. 


\section{TABLE 1}

\section{SOME PARAMETERS FOR THE VON KARMAN VORTEX STREET COMPUTATIONAL, RUNS}

\begin{tabular}{|l|lclll|}
\hline Obstacle & Re & $\begin{array}{l}U_{\infty} \\
(\mathbf{c m} / \mathbf{s})\end{array}$ & $\begin{array}{l}d \\
(\mathbf{c m})\end{array}$ & $\begin{array}{l}L \times H \\
(\mathbf{c m} \times \mathbf{~ c m})\end{array}$ & $\begin{array}{l}\text { Mesh } \\
\text { Resolution }\end{array}$ \\
\hline \hline Rectangular Block & 30 & 2.3 & 2 & $24 \times 20$ & $44 \times 44$ \\
\hline Circular Cylinder & 131 & 10.0 & 2 & $24 \times 20$ & $80 \times 96$ \\
\hline Circular Cylinder & 1000 & 76.5 & 2 & $24 \times 20$ & $80 \times 96$ \\
\hline Flat Plate & 10000 & 76.5 & 20 & $240 \times 200$ & $80 \times 96$ \\
\hline
\end{tabular}

The HMS code provides an option of two different numerical advection schemesthe first-order donor-cell scheme and the second-order slope-limiting van Leer scheme (Ref. 3). Scoping calculations performed with the donor-cell method showed that even at sufficiently high Reynolds numbers, no sustained vortex street occurred because the excessive amount of numerical diffusion inherent in the method damped out the physical flow oscillations. Using the less diffusive van Leer method alleviated this numerical damping problem. Results discussed in subsections $C, D$, and $E$ were obtained with the van Leer convection scheme.

In all calculations for the von Karman vortex street problem, we chose the size of the time steps to satisfy the Courant criterion for numerical stability. Table 1 summarizes the other relevant parameters for the computational runs discussed below. We also performed additional runs (not reported here) at the same Reynolds numbers as those used in the cases to be discussed, but with a different combination of $U_{\infty}$ and $d$. The calculations were carried out to verify-as they did-that the flow pattern and Strouhal number for a particular problem depend solely on the Reynolds number, as predicted from dimensional analysis.

\section{Flow Past Rectangular Block}

Experimental data available for this problem are not as extensive as those for flow past a cylinder. Therefore, only one calculation, in which $\operatorname{Re}=30$, is presented here. Experimental observation has indicated that no vortex street occurs in this low-speed flow. (Recall that the critical Re for vortex-street formation is $\sim 40$.) We have also performed runs at higher Reynolds numbers and obtained flow patterns with vortex 
streets similar to those for flows around a flat plate and cylinder (presented in the next two subsections).

Figure 4 shows the $44 \times 44$ variable mesh used for this calculation and the rectangular block represented by mesh obstacle cells. The smallest cell is $0.25 \mathrm{~cm} \times 0.25$ $\mathrm{cm}$, and the largest is $1.7 \mathrm{~cm} \times 1.7 \mathrm{~cm}$. The block is $2 \mathrm{~cm}$ high and has a thickness of $1 \mathrm{~cm}$. Table 1 shows values of other run parameters, such as the free stream velocity and size of the computational domain. The calculation was run to $80 \mathrm{~s}$ in problem time. At $80 \mathrm{~s}$ the calculation showed that steady state has not been reached. However, timehistory plots up to $80 \mathrm{~s}$ (see Fig. 6) indicate that the trend of approaching the steady state has been established at this time.

Both experimental observations and computed results indicate that the initial flow perturbations die out after some time, resulting in a steady flow pattern. Figure 5 shows a velocity-vector plot of the entire flow field at $80 \mathrm{~s}$. It can be seen that no vortex street occurs, and that a recirculation region is found behind the block. Note the existence of a pair of weak, elongated, counter-rotating eddies lying symmetrically about the flow centerline. Figure 6 shows the time history of the normal and streamwise velocity components at two different locations. In both plots, decaying oscillations indicate that the flow is becoming steady. The streamwise velocity time history (lower plot), taken at a location at a distance of twice the block height behind the block, shows a steady-state value of about $-0.57 \mathrm{~cm} / \mathrm{s}$. This location is a point in the region separating the two counter-rotating eddies where there is back flow.

\section{Flow Past Circular Cylinder}

The flow past a circular cylinder is a classical fluid-dynamics problem that has been investigated extensively. For the purposes of this report, we have summarized the available experimental data from the literature, as presented by Chang (Ref. 4). Data from experiments performed by Roshko (Ref. 5) and others show that the flow is stable in the range of Reynolds numbers between 40 and 150. In this regime, the nondimensional vortex shedding frequency, as given by the Strouhal number, increases rather rapidly with $\mathrm{Re}$, and an empirical correlation of $\mathrm{St}$ as a function of Re is available.

This correlation is $\mathrm{St}=0.212\left(1-\frac{21.2}{\operatorname{Re}}\right)$. The intermediate region of $150<\operatorname{Re}<300$ is the transition range where, due to irregularity of vortex shedding, no frequency law is found. The wake flow in the range of $300<\operatorname{Re}<10000$ is less regular, but St remains constant at 0.21 . Two calculations are discussed below-one at $R e=131$ and the other at $\operatorname{Re}=1000$. 
For this problem, a $80 \times 96$ uniform mesh was used (shown in Fig. 7). Each cell is $0.25 \mathrm{~cm} \times 0.25 \mathrm{~cm}$. Mesh obstacle cells were used to represent the cylinder with a diameter of $2 \mathrm{~cm}$. The free stream velocity was $10 \mathrm{~cm} / \mathrm{s}$ for $\mathrm{Re}=131$ and $76.5 \mathrm{~cm} / \mathrm{s}$ for $\operatorname{Re}=1000$. The low-Re calculation was run out to $10 \mathrm{~s}$, while the problem time for the high-Re calculation was only $1 \mathrm{~s}$ because the speed of the flow was higher. Other run parameters can be found in Table 1 .

Figure 8 is a velocity-vector plot of the computed solution for $\operatorname{Re}=131$ at $10 \mathrm{~s}$. It can be seen that the flow pattern is quite different from that shown in Fig. 5. In Fig. 8, a vortex street is evident. Figure 9 shows the time-history plots of the normal and streamwise velocity components at a point half a diameter behind and 3 diameters below the center of the cylinder. A very regular oscillatory flow pattern with the same frequency is seen in both plots. From these plots, we determine the vortex shedding frequency and obtain a St of 0.22 . The empirical correlation mentioned above gives a St of 0.18 at $\operatorname{Re}=$ 131. Therefore, the discrepancy between the calculated and experimental values of $\mathrm{St}$ is $22 \%$.

Evidence of a double row of yortices also can be found in Fig. 10, which shows the time-history plot of the velocity components at a location half a diameter behind and near, but slightly above, the centerline of the cylinder. The normal velocity plot shows the same regular oscillation seen before. However, the streamwise velocity plot shows an additional oscillation with a smaller amplitude that is out of phase with the dominant oscillation. This two-wave pattern occurs only near the centerline, where both rows of vortices contribute. The large oscillations are due to the top row of vortices while the small oscillations are due to the bottom row. There should be no ambiguity in determining the $\mathrm{St}$, as it is based on the vortex-shedding frequency from only one side (either the top or the bottom) of the cylinder.

Results for the case of $\operatorname{Re}=1000$ are shown in Figs. 11 and 12, respectively, for the flow field-vector plot and the velocity time-history plots. The effect of the initial perturbation can be seen in Fig. 12, before about $0.2 \mathrm{~s}$. However, the flow then quickly adjusts itself to the steady oscillatory flow pattern as shown. The plot shown in Fig. 11 indicates that a vortex street also exists in this case. Note that more vortices occur here (as compared to Fig. 8), confirming that the vortex-shedding frequency is higher at larger Reynolds numbers. The computed Strouhal number is 0.25 , which, when compared with the literature value of 0.21 , is in error by $19 \%$. 


\section{E. Flow Past Flat Plate}

The last von Karman vortex street problem in this report is for flow past a flat plate placed at a right angle to the oncoming flow. Experimental data abound for this case. However, we have considered a case in which Re is fairly high. Data in the literature show that in the range $6000<\operatorname{Re}<600000$ the Strouhal number is independent of Re (see Abernathy [Ref. 6]). In this range, Abernathy's experimental data indicate that $\mathrm{St}=$ 0.161 for a constriction ratio (ratio between channel height and plate height) of 13.98. Recall that the constriction ratio is 12 for all the calculations discussed.

The present calculation is at $\operatorname{Re}=10000$, and the run parameters are given in Table 1. The mesh used here is the same as that used in the case of flow over a cylinder. The $20-\mathrm{cm}$-high plate is modeled by an internal wall (infinitely thin) that is eight computational cells in height. The high Re in this case is achieved by a large value of $d$. The free-stream velocity is $76.5 \mathrm{~cm} / \mathrm{s}$. This calculation was run to a problem time of $15 \mathrm{~s}$.

Figure 13 is a plot of the computed flow field at a time of $6 \mathrm{~s}$. The double row of vortices are clearly evident in the figure. Time-history plots of the normal velocity component at two different locations are shown in Fig. 14. The plot at the top shows the velocity at a point in the wake region close to the plate and shows a fairly regular oscillatory pattern. The plot at the bottom is for a point farther away from the plate and shows a less regular pattern. Velocity-time-history plots (not shown) taken at locations still farther (e.g., over four plate heights) from the plate exhibit a highly chaotic pattern characteristic of fully developed turbulent flows. The Strouhal number was calculated to be 0.185 , which is $15 \%$ higher than the experimental value of 0.161 .

Note that the calculated Strouhal number in this case, as well as in the flows past a block and a cylinder reported above, is about $10-20 \%$ higher than the experimental values found in the literature. A factor contributing to this discrepancy is the constriction ratio, $H / d$, which we have assumed to be 12 in all the calculations but could be much higher in the actual wind tunnel experiments. The data in Ref. 6 indicate that St decreases as the constriction ratio increases. Widening and lengthening the computational flow channel and increasing the resolution of the mesh, particularly around the flow obstacle, may improve the accuracy of the calculations. 


\section{SANE:A FLAME FACILITY TEST PROBLEM}

\section{A. Background}

The Flame Acceleration Measurement and Experiments (FLAME) Facility (Ref. 7) is a large horizontal rectangular channel made of heavily reinforced concrete that was designed and built by the Sandia National Laboratories for the U.S. Nuclear Regulatory Commission. The dimensions $-1.83 \mathrm{~m}$ wide, $2.44 \mathrm{~m}$ high, and $30.5 \mathrm{~m}$ long, giving a total volume of $136 \mathrm{~m}^{3}$-were selected to be half-scale of the upper plenum region of an icecondenser pressurized-water-reactor (PWR) containment. The upper plenum of the icecondenser PWR containment is an annular region of rectangular cross section extending about $350^{\circ}$ around the containment. Thus FLAME differs from the upper plenum because it is straight rather than curved. The main use of the facility was to study flame acceleration and deflagration-to-detonation transition of premixed hydrogen-air compositions.

A schematic diagram of the FLAME channel showing its plan (top, side, and end views) is presented in Fig. 15, which shows the three ports along the len ${ }_{\varepsilon}$ th of the channel through which hydrogen was injected. The direction of hydrogen injection is horizontal, as shown in the top- and end-view diagrams. Plastic bags were attached to one end of channel to take up the volume gained by addition of the hydrogen. Expansion of the bags allowed the experimenters to visualize the hydrogen injection process.

In most experiments, hydrogen was injected into the channel until the desired amount was reached. Air-driven mixing fans along the channel walls were used to give a homogeneous mixture of hydrogen and air. The premixed gas at the prescribed hydrogen concentration was then ignited at one end of the channel to study flame acceleration and detonation behaviors. In one experiment, F-21, the air supply to the mixing fans was cut off because an error occurred in preparing the test. Hydrogen was injected near the bottom into the test chamber until the hydrogen occupied $13 \%$ of the entire volume. Thirty minutes after the injection of hydrogen, analysis of gas samples taken at the upper locations indicated a hydrogen volume fraction of $15 \%$, whereas gas samples at the lower locations had a hydrogen volume fraction of $10 \%$, with an uncertainty of about $0.5 \%$ in the measurement. The Sandia personnel speculated that a hydrogen plume probably developed and without the aid of mechanical mixing fans, stratification of hydrogen concentration could have developed.

The focus of all Sandia FLAME experiments was on flame acceleration and transition from deflagration to detonation. Temperature and pressure were measured 
during flame propagation. Hydrogen was injected to introduce the correct amount of the gas into the channel before the hydrogen-mixture was ignited. In a normal experiment, the hydrogen was well mixed in the air by mixing fans. Therefore, no extensive measurement of hydrogen concentration was made other than analysis of gas samples taken at a lower and an upper location in the channel before the ignition. Test F-21 was interesting because the hydrogen stratified after the mix.ng fans failed to operate. It was chosen to assess the HMS code's prediction of hydrogen mixing and the resulting level of stratification as a function of time. However, only twc experimental data points are available for comparison with the calculations-the volume fraction of hydrogen at $0.3 \mathrm{~m}$ above the channel floor and at $0.3 \mathrm{~m}$ below the channel ceiling (or $2.14 \mathrm{~m}$ from the channel floor), which were $10 \%$ and $15 \%$, respectively, at $30 \mathrm{~min}$ after the end of the hydrogen injection.

\section{B. HMS Model of FLAME Facility}

Hydrogen was injected transversely at one side of the channel through three 2.54$\mathrm{cm}$ diameter holes that are widely separated along the length of the channel, as shown in Fig. 15. To preserve the hydrogen jet momentum and dynamics, we must model a small opening with an area equivalent to a $2.54-\mathrm{cm}$-diameter hole in a computational volume that is $30.5 \mathrm{~m}$ long, $2.44 \mathrm{~m}$ high, and $1.83 \mathrm{~m}$ wide. To optimize computational rescurces, we modeled only one-sixth of the length of the channel. Symmetry was assumed about a plane cutting through the center of any one of the three injection tholes as well as about a plane halfway between any two adjacent injection ports. These planes of symmetry are shown as dotted lines in the side view of the channel depicted in Fig. 15 , in which the shaded areas indicates the part being modeled. Therefore, the computational volume has a cross section of $1.83 \mathrm{~m}$ (width) times $2.44 \mathrm{~m}$ (height) and is $5.08 \mathrm{~m}$ in the third dimension, which has been reduced by a factor of six because of the symmetry assumption.

The 3D computational domain is illustrated in Fig. 16. In the coordinate system, $X$ represents the vertical direction, $Y$ represents the longitudinal direction, and $Z$ represents the transverse direction. Therefore the $X-Z$ plane represents the cross section of the channel, with hydrogen injected in the Z-direction. Figure 17 shows the mesh of the $X-Z$ plane, indicating the location where hydrogen is injected. Table 2 gives the location of grid lines in all three dimensions. The number of computational cells is 16 in the X-direction, 15 in the $\mathrm{Y}$-direction, and 10 in the Z-direction, giving a total of 2,400. Because many operations in the HMS code have been arranged to take advantage of 
TABLE 2

LOCATION OF THE X-, Y-, AND Z-GRID LINES IN THE HMS MODEL OF THE SANDIA FLAME FACILITY

$\begin{array}{rrrr}\begin{array}{c}\text { Grid } \\ \text { Line } \\ \text { Number }\end{array} & \begin{array}{c}\text { Position of X- } \\ \text { or Vertical } \\ \text { Grid Line (cm) }\end{array} & \begin{array}{c}\text { Position of Y- } \\ \text { or Longitudinal } \\ \text { Grid Line (cm) }\end{array} & \begin{array}{r}\text { Position of Z- } \\ \text { or Transverse } \\ \text { Grid Line (cm) }\end{array} \\ 1 & 0.000 & 0.000 & 0.00 \\ 2 & 8.001 & 1.125 & 18.00 \\ 3 & 14.175 & 3.375 & 36.00 \\ 4 & 18.585 & 6.525 & 54.00 \\ 5 & 21.735 & 10.935 & 72.00 \\ 6 & 23.985 & 17.109 & 90.00 \\ 7 & 27.135 & 25.753 & 108.00 \\ 8 & 31.545 & 37.854 & 126.00 \\ 9 & 37.719 & 54.795 & 144.00 \\ 10 & 46.363 & 78.513 & 162.00 \\ 11 & 58.464 & 111.718 & \\ 12 & 75.405 & 158.206 & \\ 13 & 99.123 & 222.288 & \\ 14 & 132.328 & 314.403 & \\ 15 & 169.499 & 411.202 & \\ 16 & 206.670 & 508.000 & \\ 17 & 243.840 & & \end{array}$

vectorization on $X-Y$ planes of data, having the most number of cells in the $X$ - and $Y$-directions is computationally more efficient.

\section{Calculation Results and Comparison with Experiment}

The calculation began with the injection phase. First, the computational volume was initialized with stagnant air at $27^{\circ} \mathrm{C}$ and $1 \mathrm{~atm}$. Hydrogen was then injected as a horizontal jet in the transverse or Z-direction about $23 \mathrm{~cm}$ from the boitom of the channel at a velocity of $28 \mathrm{~m} / \mathrm{s}$ for a duration of $487 \mathrm{~s}(8.1 \mathrm{~min})$ to obtain an average airhydrogen mixture with $13 \%$ hydrogen by volume. The mixing phase began at $487 \mathrm{~s}$, 
when the jet was then shut off, and lasted for $1800 \mathrm{~s}$ ( $30 \mathrm{~min}$ ) until $2287 \mathrm{~s}$, corresponding to the time when gas samples were taken for analysis and ignition occurred. The calculation ended before the ignition.

In the calculation, the hydrogen jet was injected through a rectangular opening with a cross section of $2.25 \mathrm{~cm} \times 1.125 \mathrm{~cm}$, which has an area equivalent to half that of the 2.54-cm diameter hydrogen injection port in the experiment. Therefore, the (full) jet Reynolds number is $4.6 \times 10^{4}$. The automatic time-step-control option of the code was turned on to determine dynamically the time-step size for the calculations. For the injection phase, the time step was controlled by the Courant limit because of the high injection velocity. We used 297132 cycles for the $487 \mathrm{~s}$ of the injection phase calculation, giving an average time step size of $1.64 \times 10^{-3} \mathrm{~s}$. For the $1800 \mathrm{~s}$ mixing phase calculation, the time step was largely determined by the diffusion limit, and the total number of cycles was 16606 , giving an average time-step size of $0.108 \mathrm{~s}$.

For this problem, the specified kinematic viscosity was $0.153 \mathrm{~cm}^{2} / \mathrm{s}$. The binary mass diffusivity of hydrogen in air was specified as $0.754 \mathrm{~cm}^{2} / \mathrm{s}$, a value that is estimated from the kinetic theory of gases at low pressures. (The measured mass diffusivity of hydrogen in nitrogen at $20^{\circ} \mathrm{C}$ and $1 \mathrm{~atm}$ is $0.784 \mathrm{~cm}^{2} / \mathrm{s}$ [Ref. 8]. If air were pure nitrogen, the calculation would underpredict the molecular mixing rate because it would have used a lower mass diffusivity.) Both the viscosity and the mass diffusivity were assumed to be constant throughout the calculations. The algebraic turbulence model was used to account for turbulent mixing. The other two turbulence models, which are more advanced and computationally more intensive, were not used in this problem. These models will be assessed separately with experiments that provide extensive measurement of velocity and calculation. For the algebraic turbulence model, the most important parameter required is the turbulence length scale, which was set to $50 \mathrm{~cm}$ in the calculations. The length of this scale was selected to represent the size of the large eddies in the flow, which are responsible for most of the turbulent transport of mass and momentum. As discussed in the following, the calculated results were not very sensitive to the magnitude of the chosen turbulent length scale because mixing was largely achieved by molecular diffusion.

Figure 18 is the velocity-vector plot in the channel cross section at the injection plane (i.e., $X-Z$ plane at $Y=0) 100 \mathrm{~s}$ after the injection begins. Velocity-vector plots at other times during the injection phase basically look the same. Note that because of its buoyancy, the hydrogen jet does not penetrate very far into the channel before it turns upward to the ceiling. The buoyant plume at $100 \mathrm{~s}$ also can be visualized by means of contours of hydrogen volume fraction, as shown in Fig. 19. The injection location and 
distances in terms of equivalent jet diameters are shown, as well. Figure 20 shows the hydrogen contours at $200 \mathrm{~s}$. Note the gradual expansion of the plume. Figures 21 and 22 show the velocity-vector plot and the hydrogen-volume-fraction contour at $487 \mathrm{~s}$, the instant immediately after the injection has ended.

The next set of figures show calculated results in the mixing phase. Figure 23 shows the calculated profile of the hydrogen mole fraction as a function of distance from the bottom of the channel at two time instants. At $100 \mathrm{~s}$ into the mixing phase (i.e., after the injection has stopped), the minimum hydrogen-volume fraction is about $4 \%$ at the bottom and the maximum is about $17 \%$ at the top (dotted curve). The level of stratification is gradually decreased by diffusion. At $1800 \mathrm{~s}$ ( $30 \mathrm{~min})$, the minimum volume fraction is $9.15 \%$ and the maximum $15.44 \%$ (solid curve). The experimental measurements-10\% at a lower location and $15 \%$ at a upper location-compare well with the respective calculated volume fractions of $9.8 \%$ and $15.4 \%$. The reported uncertainty in the experimental measurement was $0.5 \%$ (in absolute hydrogen volume fraction), which is shown in the figure. It can be seen that the calculated and experimental results fall within the uncertainty of the measurement.

The calculated profile of the hydrogen volume fraction at $1800 \mathrm{~s}$ (dashed curve) with no turbulent diffusion is plotted in Fig. 23. Because the mixing phase does not involve mecharical mixing (i.e., no stirring action due to fans), results of calculations with and without a turbulent diffusion are roughly the same, as indicated in the figure. However, comparing the solid curve and dashed curves shows that the calculation with turbulent diffusion gives a slightly more uniform hydrogen distribution than the calculation with only molecular diffusion. The turbulence length scale in the turbulence model was varied from 50 to $91 \mathrm{~cm}$ in the calculations with negligible differences in the results; therefore, most of the mixing was achieved by the molecular diffusion process.

The calculated verical profiles of the hydrogen volume fraction presented in Fig. 2.3 are taken from a location at the center of the horizontal computational plane. Where the profile is taken exactly does not matter because calculations show that the gas is roughly well-mixed horizontaily after a short time into the mixing phase. This can be seen from Fig. 24, which shows contour plots of the hydrogen mole fraction at $117 \mathrm{~s}$ at three vertical or $X-Z$ planes (at $Y=0,0.5,4.6 \mathrm{~m}$ ). Figure 25 depicts the hydrogen contours at the plane of injection $1800 \mathrm{~s}$ into the mixing phase, showing the stratification of hydrogen concentration vertically. Note that the contour lines are perfectly straight. Similar plots at any other vertical planes show identical results, so the hydrogen distribution on any horizontal plane is uniform at this time. Figure 26 shows the timehistory ploi of the computed hydrogen mole fraction at several locations in the channel. 
Note that the curves for the two locations at the same height (first of the three indices indicating cell location) merge only a short time into the mixing phase.

\section{HDR CONTAINMENT FACILITY TEST PROBLEM}

\section{A. Background}

The Heiss Dampf Reaktor (HDR) building (Ref. 9) near Frankfurt, Germany, is a full-scale containment facility designed to provide experimental data on simulated reactor accident situations. The containment consists of a steel shell that is $60 \mathrm{~m}$ high and $20 \mathrm{~m}$ in diameter and has a free volume of $11300 \mathrm{~m}^{3}$. This steel shell is surrounded by a secondary concrete shell, with a annular gap between. Vertical cross-sectional views of the containment at two angles, presented in Fig. 27, show the spiral and standard stair cases. The HDR facility has a multicompartmental geometry (72 compartments) with a large amount of steel structures (total interior steel surface area in excess of $15000 \mathrm{~m}^{2}$ ) and a very large dome with a volume of about $5000 \mathrm{~m}^{3}$. Overall, about 200 passageways interconnect the compartments.

The experiment chosen for the code assessment discussed in this document, Test T31.5, is a large-break LOCA and a hydrogen distribution experiment that lasted for about an hour. This experiment was selected as one of the OECD/CSNI International Standard Problems and was called ISP-23.

In the experiment, a blowdown of the HDR pressure vessel occurring for $50 \mathrm{~s}$ injected $30 \mathrm{Mg}$ ( $1 \mathrm{Mg}=1000 \mathrm{~kg}$ ) of steam-water mixture into the containment. (The flow Reynolds number at the peak blowdown rate was $2.1 \times 10^{7}$.) Then a superheated steam release at a rate of $2.2 \mathrm{~kg} / \mathrm{s}\left(\mathrm{Re}=2.8 \times 10^{6}\right)$ occurred roughly between $21 \mathrm{~min}$ and $36 \mathrm{~min}$ after the blowdown began. The superheated steam release was followed immediately by a release . ilight gas (15\% hydrogen and $85 \%$ helium on a volume basis) at $0.24 \mathrm{~kg} / \mathrm{s}$ $\left(\operatorname{Re}=1.9 \times 10^{5}\right)$ from $36 \mathrm{~min}$ to $48 \mathrm{~min}$ after the beginning of the blowdown. For safety reasons, hydrogen was diluted by helium to form the light gas in this experiment to reduce the flammability of the gas mixture. A time sequence of the these events in the experiment is presented in Table 3. The blowdown and gas releases were at about the $22-\mathrm{m}$ height level. Figure 28 shows (a) a vertical, cross-sectional view of the containment showing the room where the blowdown and gas releases took place, and (b) details of the blowdown and gas release pipes. The blowdown pipe has a diameter of $43.5 \mathrm{~cm}$ and is pointed nearly vertically upward, while the gas release pipe is $8 \mathrm{~cm}$ in diameter and is directed inward horizontally. Pressure, temperature, and volume fractions of air, steam 
TABLE 3

Time Sequence of Events in the HDR T31.5 Experiment

\begin{tabular}{|c|c|}
\hline TIME & EVENT \\
\hline \multirow{2}{*}{$0 \mathrm{sec}$} & \\
\hline & $\begin{array}{c}\text { BLOWDOWN (simulating LBLOCA) } \\
30 \mathrm{Mg} \text { total }\end{array}$ \\
\hline & (NO SOURCE INJECTION) \\
\hline \multirow{2}{*}{$36 \min$} & $\begin{array}{l}\text { SUPERHEATED STEAM RELEASE } \\
2 \mathrm{Mg} \text { total }\end{array}$ \\
\hline & $\begin{array}{l}\text { RELEASE OF LIGHT GAS MIXTURE } \\
\left(15 \% \mathrm{H}_{2}, 85 \% \mathrm{He}\right)\end{array}$ \\
\hline $48 \mathrm{~min}$ & $168 \mathrm{~kg}$ total \\
\hline
\end{tabular}

and light gas were measured at various locations as a function of time. These experimental measurements are used for assessment of the HMS calculations.

\section{B. HMS Model of the HDR Containment Facility}

The HMS code can model 3D geometries in cylindrical or rectangular coordinates. In the case of the HDR containment, using cylindrical geometry is more appropriate. The containment building is represented by a 3D mesh consisting of 9 radial cells, 30 axial cells, and 23 azimuthal cells (giving a total of 6,210 computational cells) with variable mesh spacing. The discretization of the $3 \mathrm{D}$ computational volume, given by the 
TABLE 4

DISCRETIZATION OF THE 3D COMPUTATIONAL VOLUME FOR THE HDR CONTAINMENT FACILITY

\begin{tabular}{|c|c|c|c|c|c|c|}
\hline $\begin{array}{l}\text { Cell } \\
\text { Edge } \\
\text { Number }\end{array}$ & $\begin{array}{l}\text { Position of } \\
\text { Radial Edge } \\
\text { (m) }\end{array}$ & $\begin{array}{c}\Delta \mathbf{R} \\
(\mathbf{m})\end{array}$ & $\begin{array}{l}\text { Position of } \\
\text { Axial edge } \\
\text { (m) }\end{array}$ & $\frac{\Delta Z}{(\mathrm{~m})}$ & $\begin{array}{c}\text { Position of } \\
\text { Azimuthal edge } \\
\text { (deg) }\end{array}$ & $\underset{(\mathrm{deg})}{\Delta \theta}$ \\
\hline 1 & 0.0 & & -8.00 & & 20 & 20 \\
\hline 2 & 1.5 & 1.5 & -6.80 & 1.20 & 36 & 16 \\
\hline 3 & 2.3 & 0.8 & -5.80 & 1.00 & 52 & 16 \\
\hline 4 & 3.5 & 1.2 & -4.00 & 1.80 & 68 & 16 \\
\hline 5 & 5.0 & 1.5 & -3.00 & 1.00 & 84 & 16 \\
\hline 6 & 6.5 & 1.5 & -2.00 & 1.00 & 94 & 10 \\
\hline 7 & 8.0 & 1.5 & -1.00 & 1.00 & 109 & 15 \\
\hline 8 & 9.5 & 1.5 & 1.80 & 2.80 & 124 & 15 \\
\hline 9 & 10.0 & 0.5 & 2.80 & 1.00 & 139 & 15 \\
\hline 10 & 10.6 & 0.6 & 3.80 & 1.00 & 154 & 15 \\
\hline 11 & & & 4.80 & 1.00 & 169 & 15 \\
\hline 12 & & & 6.40 & 1.60 & 184 & 15 \\
\hline 13 & & & 7.40 & 1.00 & 197 & 13 \\
\hline 14 & & & 9.00 & 1.60 & 213 & 16 \\
\hline 15 & & & 10.00 & 1.00 & 227 & 14 \\
\hline 16 & & & 12.50 & 2.50 & 241 & 14 \\
\hline 17 & & & 13.75 & 1.25 & 255 & 14 \\
\hline 18 & & & 14.75 & 1.00 & 270 & 15 \\
\hline 19 & & & 16.55 & 1.80 & 289 & 19 \\
\hline 20 & & & 17.55 & 1.00 & 305 & 16 \\
\hline 21 & & & 19.25 & 1.70 & 322 & 17 \\
\hline 22 & & & 21.00 & 1.75 & 340 & 18 \\
\hline 23 & & & 22.50 & 1.50 & 360 & 20 \\
\hline 24 & & & 25.00 & 2.50 & & \\
\hline 25 & & & 27.50 & 2.50 & & \\
\hline 26 & & & 29.50 & 2.00 & & \\
\hline 27 & & & 30.85 & 1.35 & & \\
\hline 28 & & & 34.00 & 3.15 & & \\
\hline 29 & & & 40.00 & 6.00 & & \\
\hline 30 & & & 47.00 & 7.00 & & \\
\hline 31 & & & 48.00 & 1.00 & & \\
\hline
\end{tabular}


location of the grid lines, is shown in Table 4. Cell number 7 in the radial direction, number 11 in the azimuthal direction, and number 24 in the axial direction receive the steam and light-gas sources. Figure 29 shows the computational mesh on vertical and horizontal planes.

A horizontal cross section of the computational model at $16 \mathrm{~m}$ and its actual physical geometry are compared Figure 30 . Figure 31 shows the same comparison for a vertical section at an azimuthal angle of $10^{\circ}$. Note that the hemispherical top of the containment is approximated in the model by a flat-head cylinder having the same volume. The annular gap between the steel and concrete shells is represented by a single layer of mesh cells. The concrete shell is not shown in the mesh but is modeled as a 1-m-thick slab surrounding the entire computational domain which can exchange heat with the annular gap. In the computational model, the shaded regions represent blocked-out (dead or inactive) computational cells, whereas line segments positioned on cell edges are inertial barriers. In the model, the blocked cells are specified by 244 mesh obstacle definitions and the flow barriers are specified by 159 wall definitions. The flow obstacles and barriers combine to model all the major floors and walls in the containment building. Even though the inertial barriers have zero thickness in the computational mesh, heat conduction calculations for these solid structures are based on an average thickness of $1 \mathrm{~m}$ for the concrete walls and floors and $3 \mathrm{~cm}$ for the outer cylindrical steel shell. In this modeling procedure, we physically represent all the compartments. However, because of the constraints inherent in an orthogonal computational mesh, the flow areas (doors, connections, and other openings) among compartments cannot be represented exactly. This constraint in the modeling capability will be discussed later in the results subsection of the calculation.

We have modeled the effects of internal structures (e.g., steel structures in various rooms in the HDR containment), too fine to be resolved by the computational mesh, on heat transfer and condensation by distributed heat sinks. Distributed heat sinks are specified by defining the total exposed surface area and average half thickness over a region of the computational space. (The half-thickness specification is used because the heat conduction calculation in these distributed heat sinks assumes symmetry at the centerline.) From the heat sink specification, the code calculates the free gas volume within the defined region and distributes the solid heat sinks evenly over all the included fluid cells. We have chosen to subdivide the HDR containment into horizontal disks extending radially from the centerline to the steel shell (radial position of $10 \mathrm{~m}$ ) and axially between the edges of each vertical computational cell. The half-thickness, 
TABLE 5

SPECIFICATION OF INTERNAL HEAT SINKS

FOR THE HDR CONTAINMENT MODEL

\begin{tabular}{|c|c|c|c|}
\hline $\begin{array}{l}\text { Disk Height } \\
\text { (m) }\end{array}$ & $\begin{array}{l}\text { Number of } \\
\text { Heat Sinks }\end{array}$ & $\begin{array}{l}\text { Total Exposed Area } \\
\qquad\left(10^{3} \mathrm{~m}^{2}\right)\end{array}$ & $\begin{array}{l}\text { Half Thickness } \\
\text { (cm) }\end{array}$ \\
\hline$-8.0<z<-6.8$ & 71 & 0.180 & 0.47 \\
\hline$-6.8<z<-5.8$ & 0 & 0.000 & 0.00 \\
\hline$-5.8<z<-4.0$ & 119 & 0.609 & 0.32 \\
\hline$-4.0<z<-3.0$ & 68 & 0.268 & 0.30 \\
\hline$-3.0<z<-2.0$ & 108 & 0.302 & 0.30 \\
\hline$-2.0<z<-1.0$ & 43 & 0.032 & 0.29 \\
\hline$-1.0<z<1.8$ & $\because 145$ & 1.383 & 0.37 \\
\hline $1.8<z<2.8$ & 99 & 0.330 & 0.37 \\
\hline $2.8<z<3.8$ & 142 & 0.342 & 0.38 \\
\hline $3.8<z<4.8$ & 60 & 0.029 & 0.36 \\
\hline $4.8<z<6.4$ & 132 & 0.684 & 0.36 \\
\hline $6.4<z<7.4$ & 88 & 0.506 & 0.28 \\
\hline $7.4<z<9.0$ & 119 & 0.431 & 0.28 \\
\hline $9.0<z<10.00$ & 75 & 0.201 & 0.64 \\
\hline $10.0<z<12.50$ & 116 & 0.838 & 0.44 \\
\hline $12.5<z<13.75$ & 101 & 0.750 & 0.44 \\
\hline $13.75<z<14.75$ & 46 & 0.212 & 1.13 \\
\hline $14.75<z<16.55$ & 96 & 0.649 & 0.52 \\
\hline $16.55<z<17.55$ & 79 & 0.620 & 0.52 \\
\hline $17.55<z<19.25$ & 78 & 0.591 & 0.39 \\
\hline $19.25<z<21.0$ & 68 & 0.383 & 0.42 \\
\hline $21.0<z<22.5$ & 90 & 0.855 & 0.52 \\
\hline $22.5<z<25.0$ & 72 & 0.549 & 0.59 \\
\hline $25.0<z<27.5$ & 119 & 0.646 & 0.71 \\
\hline $27.5<z<29.5$ & 118 & 0.392 & 0.92 \\
\hline $29.5<z<30.85$ & 0 & 0.000 & 0.00 \\
\hline $30.85<z<34.0$ & 171 & 1.784 & 0.72 \\
\hline $34.0<z<40.0$ & 177 & 1.946 & 0.72 \\
\hline $40.0<z<47.0$ & $\underline{0}$ & 0.000 & 0.00 \\
\hline Total & 2600 & 15.514 & \\
\hline
\end{tabular}


surface area, and number of surfaces of distributed heat sinks for each horizontal disk region is specified in Table 5. The entire computational model has 9075 heat transfer surfaces, including those from distributed heat sinks and from flow barriers and obstacles. For the steel distributed heat sinks, three uniformly spaced computational nodes were used for the heat conduction calculation. This coarse nodalization was used because no steep temperature gradients are expected in the steel heat sinks. For walls or flow blockages, which are thicker than the distributed heat sinks and may be steel or concrete, we used 11 equally spaced nodes.

\section{Fluid and Thermal Parameters}

In addition to the model geometry and nodalization, other parameters are needed to specify the calculation. The experiment involves five main gas species-nitrogen and oxygen from the air, water vapor, and helium and hydrogen from the light gas $\left(15 \% \mathrm{H}_{2}\right.$, $85 \% \mathrm{He}$ ). We modeled the light gas and air as two species whose thermodynamic properties are given by weighted averages of the constituent properties. (However, modeling the five gas species separately is possible in HMS, if desired.) Therefore, the transport of only three gas components (air, steam, and light gas) was calculated. The hydrogen concentration presented in the discussion of results is obtained by multiplying the calculated light-gas concentration by $15 \%$.

For molecular transport properties, the code currently uses a simplified model of one diffusion coefficient for each of the three transport equations (mass, momentum, and energy), and each coefficient is assumed to be constant throughout the calculation. In reality, the coefficient can be a strong function of temperature and gas mixture composition. For example, the viscosity (or diffusion coefficient for momentum) for gases generally increases with temperature roughly as the 0.6 to 1.0 power of the (absolute) temperature. The viscosity of a gas mixture can be determined from the semiempirical formula of Wilke (Ref. 10) with an average deviation of about $2 \%$. Such a formula shows that the dependence of mixture viscosity on composition is extremely nonlinear for some mixtures, particularly those of light and heavy gases. For mass diffusion, the situation is even more complicated. Except for a binary system, the mass diffusivity of a species into the mixture is generally different from that of another species in the mixture. Nevertheless, depending on the desired accuracy and whether diffusion processes dominate the flow dynamics, the single, constant diffusivity simplification is often quite useful. For the present calculation, $0.15 \mathrm{~cm}^{2} / \mathrm{s}$ was used for the kinematic viscosity, and $0.21 \mathrm{~cm}^{2} / \mathrm{s}$ was used for the thermal diffusivity. For the mass diffusivity, values of 0.33 and $0.75 \mathrm{~cm}^{2} / \mathrm{s}$, respectively, were used before and after 
the light gas was introduced. A higher diffusivity was used in the light-gas phase of the calculation because the mobility of hydrogen and helium molecules in air is higher than that of water molecules.

For the turbulence model, we used the algebraic model with a length scale of $1 \mathrm{~m}$. Some auxiliary calculations show that varying this length scale does not significantly change the results for this problem. The other two, more advanced turbulence models available in the code are the subgrid-scale and $k-\varepsilon$ models, which solve additional differential, transport equations. Because the current problem involves a very complicated geometry (which is modeled exactly in the computation) and the combined effects of heat transfer, condensation, and buoyancy-driven flow, we feel that the benefit be gained from using more advanced turbulence models will not be enough to justify the additional computer resources required. The turbulence models will be assessed separately against experiments that have simpler, well-characterized geometries and such extensive measurements of the flow field as velocity, temperature, and/or concentration profiles.

The calculation was started with air throughout the computational volume at a pressure of 1 bar and a uniform temperature of $32^{\circ} \mathrm{C}$. The solid structures also had an initial temperature of $32^{\circ} \mathrm{C}$. The steam and light gas were introduced at the source location at the appropriate time (see the following subsection). The boundary conditions were no-slip at all solid surfaces and periodic at the azimuthal boundaries (i.e., at $\theta=0^{\circ}$ and $360^{\circ}$ ). The initial time-step size, $0.002 \mathrm{~s}$, was adjusted automatically to satisfy criteria based on the material Courant and diffusion limits for numerical stability. The calculations proceeded with time-step sizes a little larger than the initial value. One hour of the experiment (from initiation of the blowdown) was simulated.

\section{Blowdown and Gas Release Sources}

An important part of the calculation involves an isentropic expansion model to determine the correct amount of steam received by the containment, based on the measured blowdown source data. The HDR experiment T31.5 involves a large-break blowdown of the pressure vessel followed by a superheated steam release and then by a light gas release. The large-break blowdown injected $30 \mathrm{Mg}$ of two-phase, steam-water mixture into the containment in $50 \mathrm{~s}$. Because HMS is a single-phase compressible fluid code, it is currently unable to model the two-phase critical flow mixture entering the containment. Therefore, we have developed a blowdown jet-expansion model to extract the correct mass flow rate of steam source into the containment. The liquid component 
of the blowdown and any condensate are assumed to drain into the containment sump area and are removed effectively from the containment inventory.

The blowlown source is specified by data of experimentally measured timedependent mixture pressures, temperatures, steam qualities, and mass flow rates at a location immediately upstream of the blowdown pipe exit. To calculate the actual amount of steam source received by the containment, the blowdown model assumes thermodynamic equilibrium between the vapor and liquid phases in the mixtures, both at the pipe exit and after expansion into the containment. The quality and temperature of the expanded blowdown source were computed by assuming that the expansion from the blowdown pipe exit pressure and quality to the containment pressure is an isentropic (reversible, adiabatic) process. The procedure is illustrated in Fig. 32 on a schematic temperature-entropy phase diagram for water. From the specified blowdown jet pressure, $P_{j e t}$, and quality, $x_{j e t}$ the mixture specific entropy, $S_{\text {mix }}$, can be determined using a table of saturated steam properties that is built into the code

$$
S_{\text {mix }}=S_{f, j e t}+x_{j e t} S_{f g, j e t}
$$

where $S_{f, j e t}$ is the specific entropy of the saturated liquid and $S_{f g, j e t}$ is the specific entropy of vaporization at the blowdown jet pressure $P_{j e t}$. (Here and in subsequent discussion, the subscript jet is used to indicate conditions in the blowdown jet before the expansion, which were measured in the experiment at a location immediately upstream of the blowdown pipe exit. The subscript con indicates conditions after expansion into the containment.) The entropy, $S_{\text {mix }}$, of the mixture after expansion to the containment pressure, $\mathrm{P}_{\text {con, }}$ is the same as that in the blowdown jet, and its steam quality can be calculated with the lever rule

$$
x_{\text {con }}=\left(S_{\text {mix }}-S_{f, c o n}\right) / S_{f g, c o n},
$$

where $\mathrm{S}_{\mathrm{f}, \text { con }}$ and $\mathrm{S}_{\mathrm{fg}}$,con are the respective specific entropies of the saturated liquid and of vaporization at $P_{c o n}$. Therefore, depending on its entropy $\left(S_{\text {mix }}\right)$, the two-phase mixture can either flash to generate more vapor or undergo some condensation as a result of expanding into the containment. This will be discussed further when we show the steam qualities in the blowdown jet and in the containment source.

Figure 33 shows the mass flow rates of the blowdown (measured in the blowdown pipe) for two time intervals, 0 to $5 \mathrm{~s}$ and 0 to $50 \mathrm{~s}$, with the steam and liquid components plotted separately. The peak total flow rate is about $2350 \mathrm{~kg} / \mathrm{s}$. The two-phase jet at a 
pressure of $P_{\text {jet }}$ enters the containment, which is at a pressure of $P_{\text {con }}$. The pressures are plotted for two time intervals in Fig. 34. The temperature of the steam source to the containment after the isentropic expansion process and the original blowdown jet temperature are shown in Fig. 35. Whether the blowdown jet flashes or condenses upon expansion into the containment can be seen from the steam-quality plots in the jet and in the containment source, shown in Fig. 36 . For a time roughly less than $0.5 \mathrm{~s}$, the jet actually condenses because the containment source quality is less than the original jet quality. Also, for a time greater than about $17 \mathrm{~s}$, the jet again condenses somewhat because of the higher jet quality. Between the times of $0.5 \mathrm{~s}$ to $17 \mathrm{~s}$, the jet is flashing, as demonstrated by the higher containment source quality for this time interval. Because the flashing and condensation of the two-phase blowdown jet occurs during its expansion into the containment volume, the real, individual source rates of steam and liquid water received by the containment are different from the measured values in the blowdown pipe. Figure 37 shows the calculated steam and liquid source rates to the containment after the expansion process for two time intervals. The rates can be compared with the original source data plotted in Fig. 33.

The integrated steam and liquid masses in the original jet and in the mixture after expansion into the containment demonstrate the efficiency of the blowdown process. Figure 38 shows the mass of steam in the blowdown jet and in the containment source after the jet expansion, and Fig. 39 shows the same plots for the mass of liquid. The top curve on each plot is the total mass of liquid and steam jetting into the containment. The figures show that at about $50 \mathrm{~s}$ after the blowdown begins, $30 \mathrm{Mg}$ of steam and liquid has been jetted into the containment. Of the total amount, $14 \mathrm{Mg}$ of steam and $16 \mathrm{Mg}$ of liquid have entered the containment by adiabatic expansion, which can be compared with the $12 \mathrm{Mg}$ of steam and $18 \mathrm{Mg}$ of liquid in the blowdown jet. Because HMS currently does not keep track of the liquid inventory, the liquid component of the source immediately disappears from the calculation. Any steam that condenses on walls, floors, and/or internal heat sinks is also assumed to disappear from the calculation. The amount of steam in the containment for two time intervals is plotted in Fig. 40. The upper curve on each plot is the total steam mass source, and the lower curve is the actual amount of steam in the containment. The difference between the two curves gives the amount of steam that has been condensed. At roughly $50 \mathrm{~s}$, there is about $7.1 \mathrm{Mg}$ of steam in the containment. Comparison with the total source of $14.4 \mathrm{Mg}$ means that $7.3 \mathrm{Mg}$ of steam has condensed on solid surfaces within the containment.

Figure 41 shows the pressures, temperatures, and mass flow rates for the superheated steam and light-gas releases (during the time interval from 1260 to $2880 \mathrm{~s}$ 
or 21 to $48 \mathrm{~min}$ ) measured in the injection pipe. The data for the steam release show that the average temperature was around $128^{\circ} \mathrm{C}$ and that the pressure was 3.8 bars. The measured temperature was substantially below the saturation temperature corresponding to the measured pressure: (saturation temperature at 3.8 bars is $142^{\circ} \mathrm{C}$ ). Therefore, according to the reported temperature and pressure, the steam could not have been superheated, as claimed in the experiment. Because the steam came directly from the boiler in a power plant next to the containment building, the presence of a significant amount of air or other gase's was unlikely. One possible explanation for the subcooled steam is the supersaturation phenomenon discussed in Ref. 11-a nonequilibrium effect due to the s'-called van der Waal's loops exhibited by isotherms on pressure-volume vapor-liquid phase diagrams. What caused the apparent contradiction between the measured thermodynamic condition that implied a subcooled state and the experimenters' claim of superheated steam is not clear. To determine the steam source rate for calculations in this superheated steam release phase, we assumed arbitrarily that the steam had a quality of $50 \%$ and was expanded isentropically from the measured pipe pressure to the calculated containment pressure. Figure 42 shows, after expansion, the calculated cumulative steam source that was introduced into the containment during the first hour of the experiment. The initial blowdown and second steam release phases are shown by the two slopes on the curve. Also shown is the computed total amount of steam in the containment. The difference between the source mass introduced into the containment and the actual mass in the containment at any instant indicates the cumulative amount of steam condensed up to that time.

Because the flow rate is small and the expansion effects are minimal during the light gas release, the pressures and temperatures in the pipe and expansion values are nearly equal. Of course, no flashing or condensation of the source would occur. The total amount of light gas released was approximately $168 \mathrm{~kg}$.

\section{E. Calculation Results and Comparison with Experiment}

Figure 43 shows the velocity vector plots at three vertical planes around the source cell location very shortly $(7 \mathrm{~ms})$ after the blowdown has begun. In these figures, the blocked-out cells are not shaded so that the shading will not masl: any portion of the vectors. Because of the impingement plate on top of the blowdown cell (see middle figure), the steam source was reflected downward. However, adjacent planes show some upward motion as the blowdown jet has gone around the impingement plate. Note that because the dome region is not directly connected to the blowdown room, some time would pass before a significant amount of steam gets there. Figures 44 and 45 
show velocity vector plots at $132 \mathrm{~s}$ or $22 \mathrm{~min}$ (within the superheated-steam injection phase) and $2400 \mathrm{~s}$ or $40 \mathrm{~min}$ (within the light-gas release phase), respectively. Here the obstacle cells are shaded to better illustrate the flow paths in these planes. The natural circulation motion in the dome caused by steam condensing on the rolder steel shell is evident in the figures. The figures also show the complicated flow patterns typical of most regions in the containment.

The computed and experimentally measured pressures at the indicated locations are plotted as a function of time in Fig. 46. Both plots show that the containment pressure rose rapidly to its peak value because of the blowdown of the pressure vessel. Then the containment depressurized because of steam condensing on internal surfaces within the steel shell. The rate of depressurization slowed down in time as the amount of steam decreased and the condensation surfaces warmed up. At $21 \mathrm{~min}$, because of the second steam injection, the pressure started increasing but at a rate much lower than that during the initial blowdown. From 36 to $48 \mathrm{~min}$, an additional increase in pressure occurred due to the light-gas injection. After the light-gas injection ended, the pressure decreased slowly because the remaining amount of steam condensed in the containment. From the figure, it can be seen that the agreement between the calculation and experimental data is very good, except for some slight overprediction after about 27 min. The peak pressure of 2.37 bars, occurring at around $40 \mathrm{~s}$, is reproduced excellently in the calculation. The pressure trace at other locations is very similar to the one shown because pressure waves are transmitted at sound speed to all interconnected volumes.

Figure 47 shows the calculated and experimental temperature time histories at three locations. The calculation overpredicted the maximum temperature by about $10^{\circ} \mathrm{C}$ both in the dome (Location A) and in a room near the bottom of the containment (Location $\mathrm{C}$ ). On the other hand, the calculated peak temperature below the blowdown pipe (Location B) agrees rather well with the data. The overprediction may be attributed to the uniform initial temperature in the calculation. Recall that the temperature in all gas volumes and solid structures were set to $32^{\circ} \mathrm{C}$ at the beginning of the calculation. This is correct only in regions around the blowdown room. It was found out, unfortunately after the calculations had been done, that the initial temperature distribution in the containment during the experiment was highly nonuniformLocations $A$ and $C$ had a temperature of about 20 to $24^{\circ} \mathrm{C}$.

Regarding the decrease of temperature after the peak value, the calculation shows a faster drop than that shown by the experimental data at Locations A and B, whereas the agreement is fairly good at Location $C$. The sharper decrease in temperature implies an overprediction of the heat-transfer coefficients at those locations. At all three 
locations, the calculation shows a period of cooling down, followed by a period of gradual temperature increase, while the experimental data indicate an overall cooling trend. At Location $B$, the experimental curve shows the abrupt temperature rise followed by a plateau region and then a sudden drop that was caused by the beginning and end of the superheated steam injection phase. This plateau region is not as evident in the calculated curve because the temperature at that time is higher than that in the data, hence the effect of introducing the superheated steam is not as distinct as in the experiment. It can be seen in all three figures that, in general, an overprediction of the temperature occurs after about 10 to $20 \mathrm{~min}$. (The slight overprediction of containment pressure at late times, as seen in Fig. 46, may be due to this overprediction in temperature.) The temperature rise in the calculation is caused by the steel internal structures, which had been heated up by the blowdown steam source, releasing their heat back to the gas. This explanation is confirmed by side calculations during the late transients in which we artificially inhibited heat transfer between the gas and steel structures and observed no heat up of the gas. In light of the overspecification of the initial temperature (for both gas and solid structures), discussed in the previous paragraph, it is not surprising that the calculated results show the early return of energy from steel structures back to gas and temperatures higher than those of the experimental data.

Figure 48 gives the measured and calculated hydrogen volume fraction at three locations. Both the calculation and experimental data show a stratification of the hydrogen concentration vertically at 1 hour from the initiation of the blowdown. The hydrogen volume fraction is roughly $2 \%$ at the top (Location A); about $1 \%$ at the middle (Location B), and less than $0.1 \%$ at the lower Location C. Agreeing with the experimental data, the calculated hydrogen concentration reaches a maximum at about 50 min, or shortly after the hydrogen injection has stopped. However, the peak value in the calculation is overpredicted $2.4 \%$ compared with slightly less than $2 \%$ in the experiment). Conversely, the calculated hydrogen volume fraction at $C$ is underpredicted at $60 \mathrm{~min}$. The experimental value is about $0.07 \%$ whereas the calculation shows an insignificant amount (roughly $10^{-4} \%$ ) of hydrogen at this location. Fig. 49 plots the volume percents of air plus light gas, of steam, and of hydrogen at the top of the dome (Location A in Fig. 48). The calculation agrees fairly well with experimental data.

It can be seen in Fig. 48 that the calculated hydrogen distribution is slightly more stratified than that observed in the experiment, that is, the concentration is overpredicted at the top and underpredicted at the bottom. The discrepancy may be explained by considering the principal hydrogen transport mechanisms-convection and (turb- 
ulent and molecular) diffusion. Hydrogen injected into containment immediately tended to rise due to motion driven by its buoyancy. This phenomenon is much like the hydrogen buoyant plume in the previous assessment problem, depicted in Fig. 20. However, because of the complicated, multicompartmental geometry in the current problem, the flow path from the injection location to the dome region is rather tortuous. From the overprediction of hydrogen concentration in the top, one may suspect that this path has been modeled with too much flow area or too little resistance, which is possible because of the relatively coarse mesh resolution used for this problem. On the other hand, penetration of hydrogen into the lower regions of the containment is mainly due to the diffusion process. Because too much hydrogen has escaped to the top in the calculation, the concentration at the bottom must be underpredicted because less hydrogen is available for the downward diffusion.

Not only the modeling of the flow paths and areas but also the accuracy of calculating the background fluid flow pattern has an effect on the hydrogen distribution. During the hydrogen injection, the containment was in a steam-condensing environment, even though the condensation rate was lower than it was immediately following the blowdown. The natural-circulation flow patterns set up by steam condensing on walls, floors, ceilings, and internal structures tend to promote mixing. However, these flows were not strong enough to counteract the buoyant motion of the hydrogen, hence stratification still occurred. (In fact, the use of water sprays in nuclear containments during severe accidents is to compound the steam condensation effect and, in addition to depressurizing the containment, to create strong eddying motions to mitigate hydrogen stratification.)

From the above results, the following suggestions can be made for future calculations, which should improve the agreement between the calculations and experimental data. First, we must have realistic representation of the initial temperature distribution throughout the containment. A single temperature for all gas volumes and solid structures is an overly simplified initial condition for this problem. The smearing of all internal steel structures over all fluid cells at each axial level, as described in Subsection B, is probably another oversimplification. Because of their importance in exchanging heat with the gas, the steel structures should be distributed more precisely in the computational model. In reviewing the computational mesh (see Figs. 29 and 45, for example), using one layer of cells to represent the air gap between the steel and concrete shells may not be adequate. Modeling this air space with at least two layers of cells will enable calculation of recirculating air flow patterns (if they exist) in both the annular region and the gap at the top. Modeling the spherical dome region as a flat 
another approximation. To model all flow paths more precisely with the correct area and resistance, a finer resolution for the mesh as a whole is needed. A strong and complicated interaction occurs between fluid dynamics and heat transfer, especially in such complex geometries as the HDR facility. Therefore, the computational model should be sufficiently accurate to capture all the expected important flow phenomena.

\section{REFERENCES}

1. T. L. Wilson and J. R. Travis, "Hydrogen Mixing Studies (HMS): Theory and Computational Model," (NUREG/CR-5948) (December 1992).

2. Th. von Karmani, "Uber den Mechanismus des Widerstandes, den ein bewegter Korper in einer Flussigkeit erzeugt," Nachr. Ges. Wiss Gottingenn, 547-556 (1912).

3. B. van Leer, "Towards the Ultimate Conservative Difference Scheme. V. A SecondOrder Sequel to Godunov's Method," J. Comp. Phys. 32, 101-136 (1979).

4. P. K. Chang, Separation of Flow (Pergamon Press, Oxford, New York, 1970), pp. 341342 .

5. A. Roshko, "On the Development of Turbulent Wakes from Vortex Streets," National Advisory Committee for Aeronautics, NACA Rep. 1911 (1954).

6. F. H. Abernathy, "Flow Over an Inclined Plate," J. Basic Engineering, September 1966, 38C-388.

7. M. P. Sherman, S. R. Tieszen, and W. B. Benedick, "FLAME Facility: The Effect of Obstacles and Transverse Venting on Flame Acceleration and Transition to Detonation for Hydrogen-Air Mixtures at Large Scale," US NRC report (NUREG/CR5275) (April 1989).

8. T. K. Sherwood, R. L. Pigford, and C. R. Wilke, Mass Transfer, McGraw-Hill, p.23 (1975).

9. L. Wolf and L. Valencia, "Results of the Preliminary Hydrogen Distribution Experiment at HDR and Future Experiments for Phase III," 16th Water Reactor Safety Information Meeting, Gaithersburg, Maryland, October 1988, 189-222, Vol. 5, (NUREG/CD-0097) (March 1989). 
10. C. R. Wilke, Ind. Eng. Chem., 18, 517-519 (1950).

11. J. O. Hirschfelder, C. F. Curtiss, and R. B. Bird, Molecular Theory of Gases and Liquids (John Wiley \& Sons, Inc., New York, 1954) p. 364. 


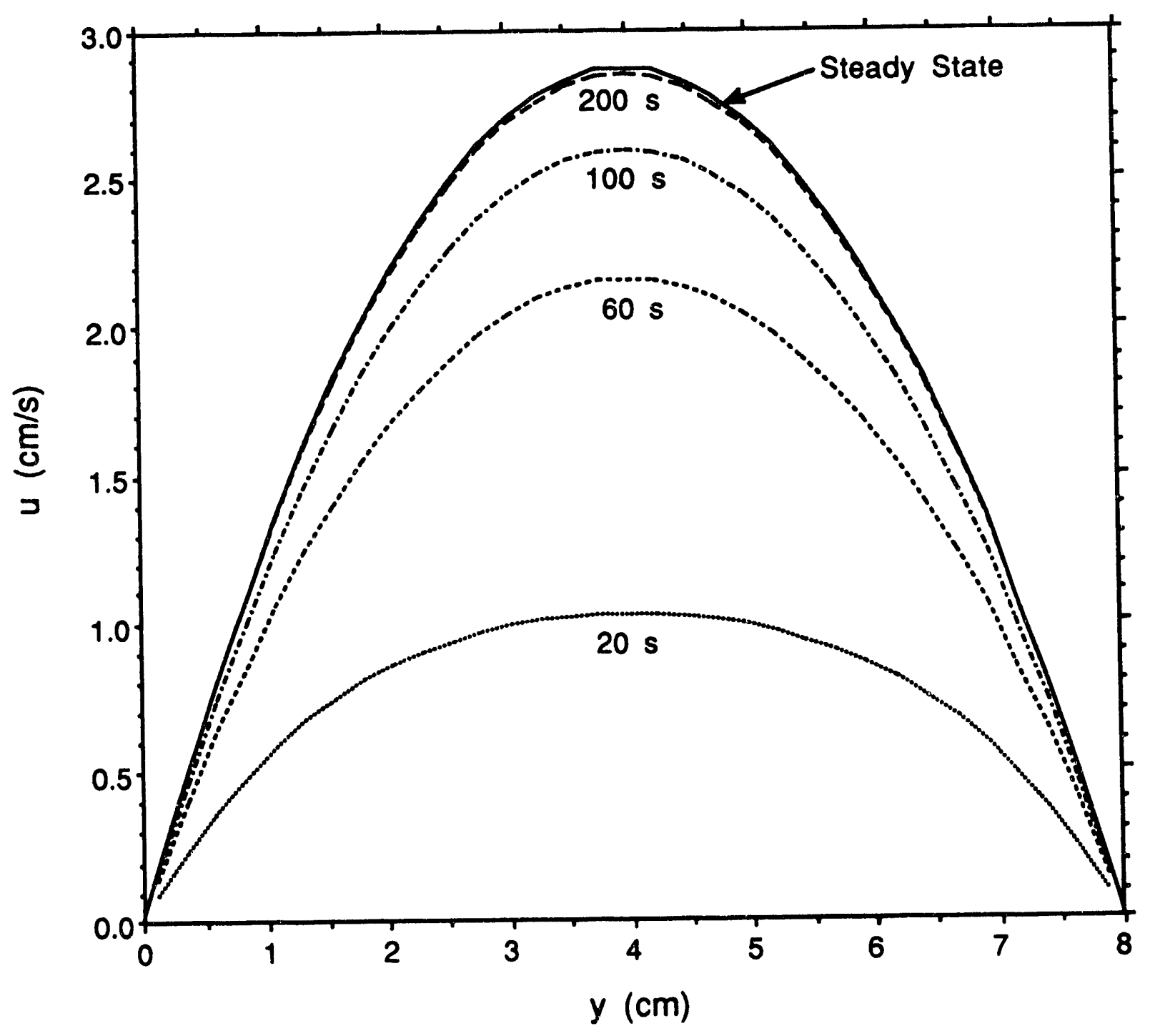

Fig. 1. Computed velocity profiles at various times showing the transient solution tending toward the steady-state distribution asymptotically as time increases for the laminar flow test problem. 


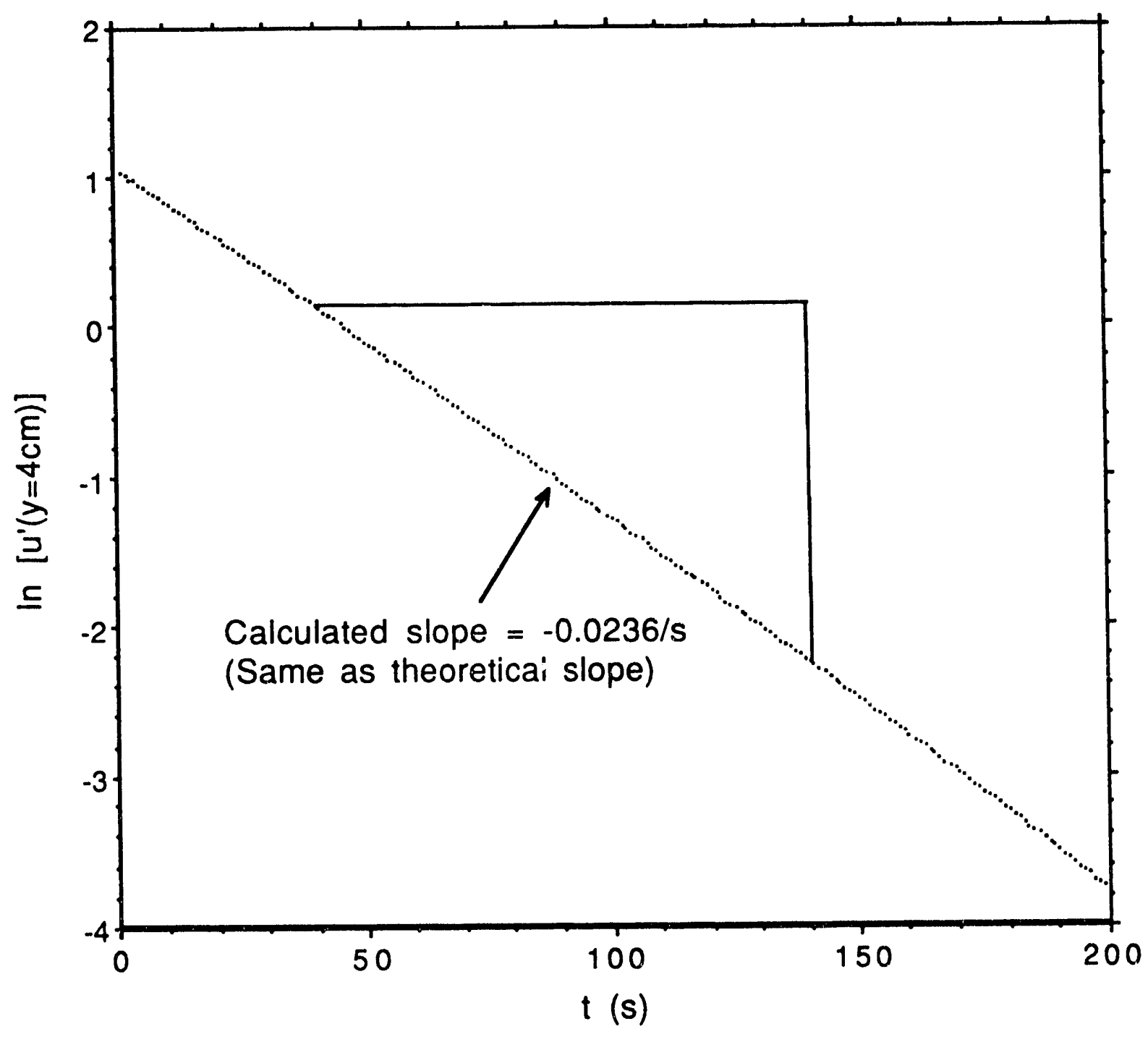

Fig. 2. Logarithm of $u^{\prime}$ at $y=4 \mathrm{~cm}$ vs $t$ for the laminar flow test problem. The straight-line plot indicates exponential decay with a decay constant given by the slope of $-0.0236 \mathrm{~s}^{-1}$, which agrees with the theoretical value. 


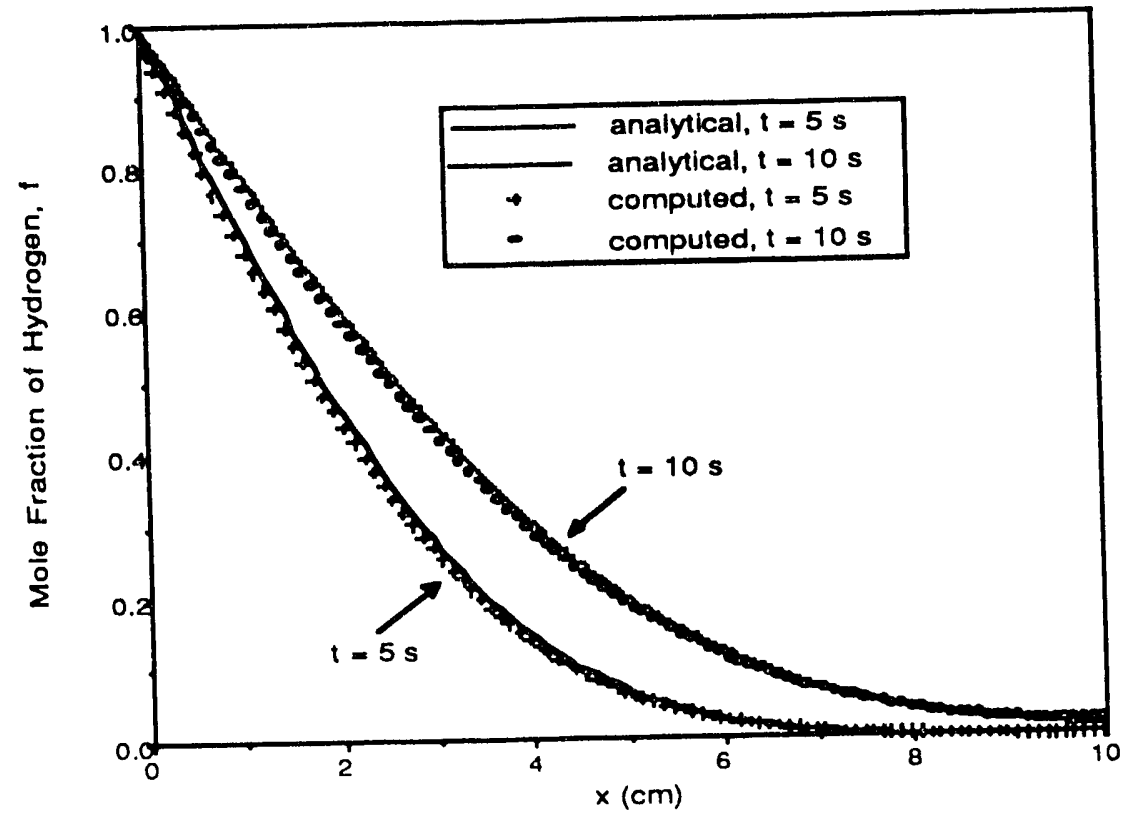

(a)

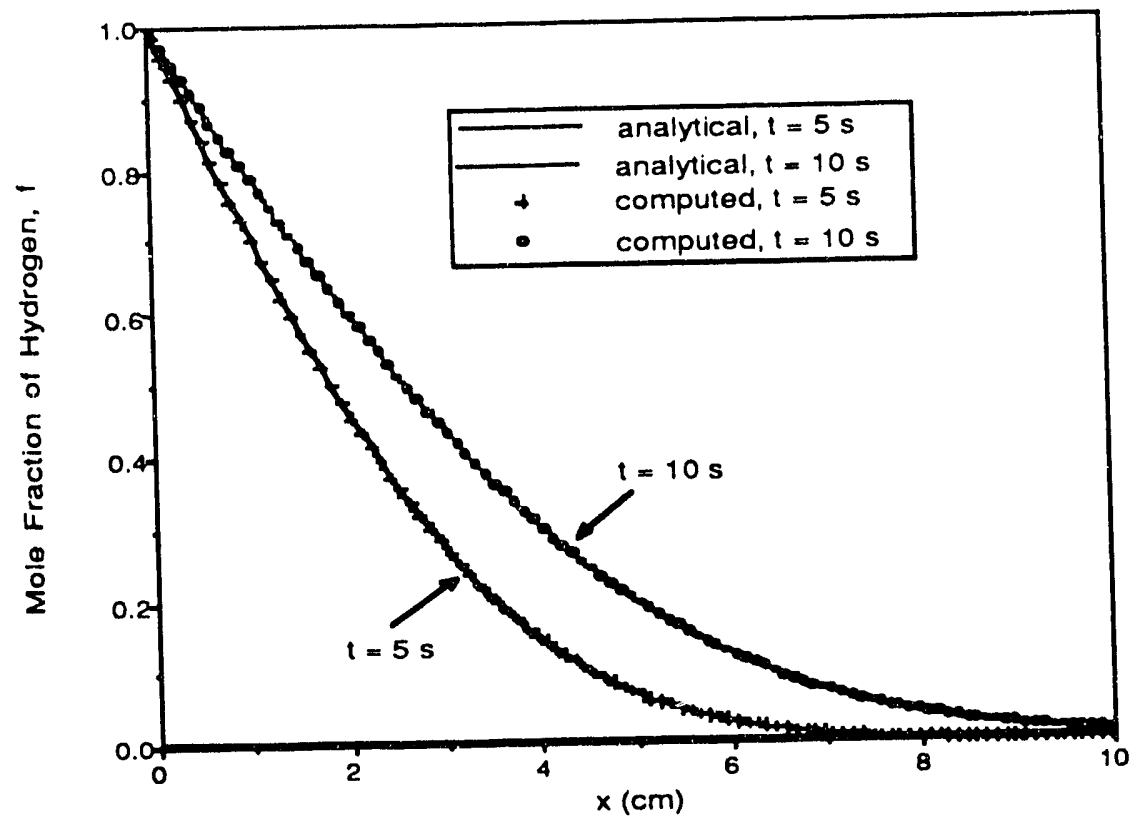

(b)

Fig. 3. Computed profiles of hydrogen mole fraction at two time instants compared with the analytical solution for the mass diffusion test problem.

(a) $\Delta x=0.1 \mathrm{~cm}$; (b) $\Delta x=0.05 \mathrm{~cm}$. 


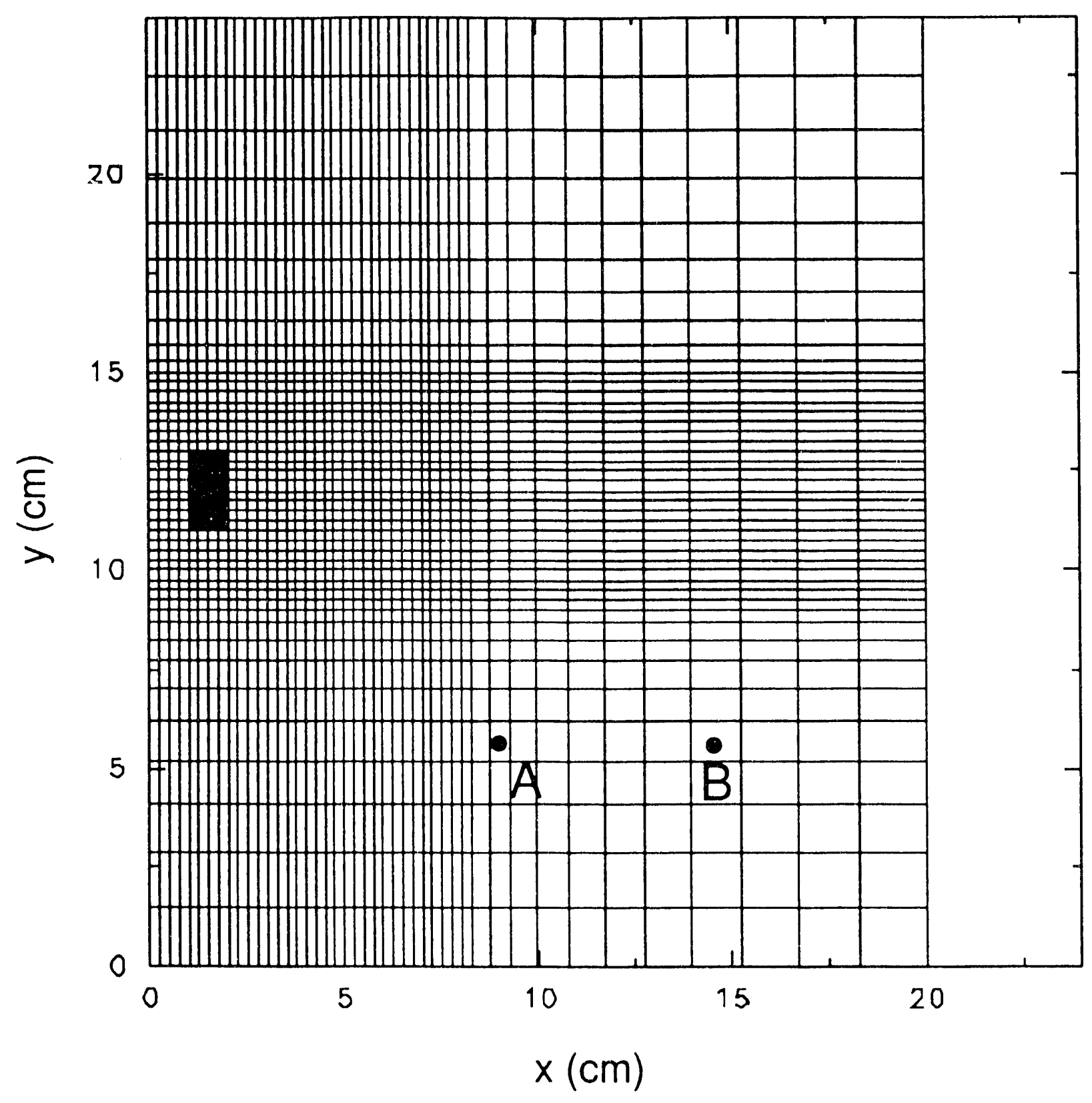

Fig. 4. Computational mesh (variable grid) used for the flow past rectangular block problem. The number of cells in $x$ and $y$ are $44 \times 44$ and the minimum and maximum cell sizes are, repectively, $0.25 \times 0.25 \mathrm{~cm}^{2}$ and $1.7 \times 1.7 \mathrm{~cm}^{2}$. Points $A$ and $B$ are locations for the velocity time-history plots in Fig. 6. 

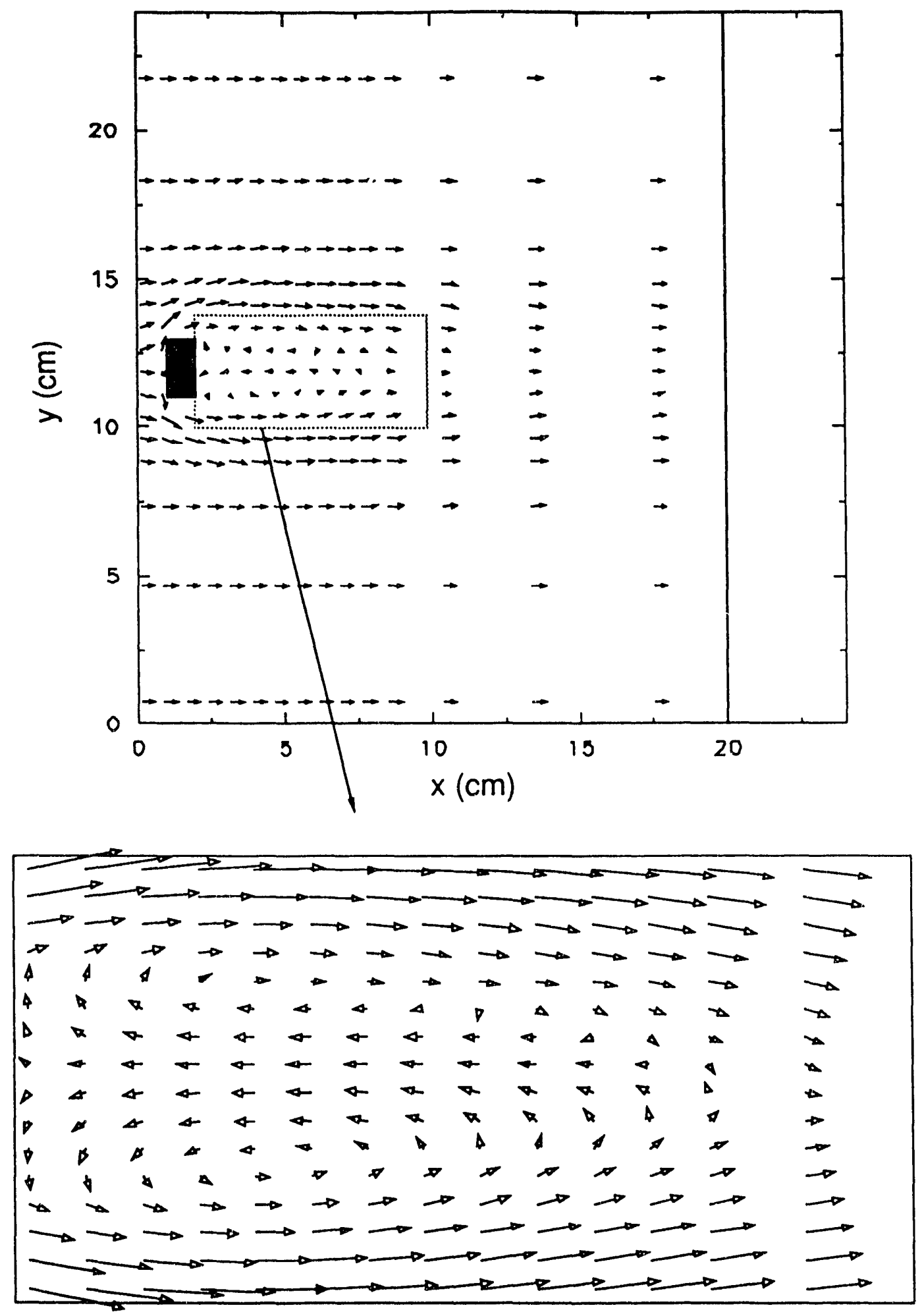

Fig. 5. Velocity vector plot for flow around a rectangular block, $\operatorname{Re}=30$, at $t=80 \mathrm{~s}$, showing nonexistence of a vortex street. Note the pair of counter-rotating eddies behind the block. 

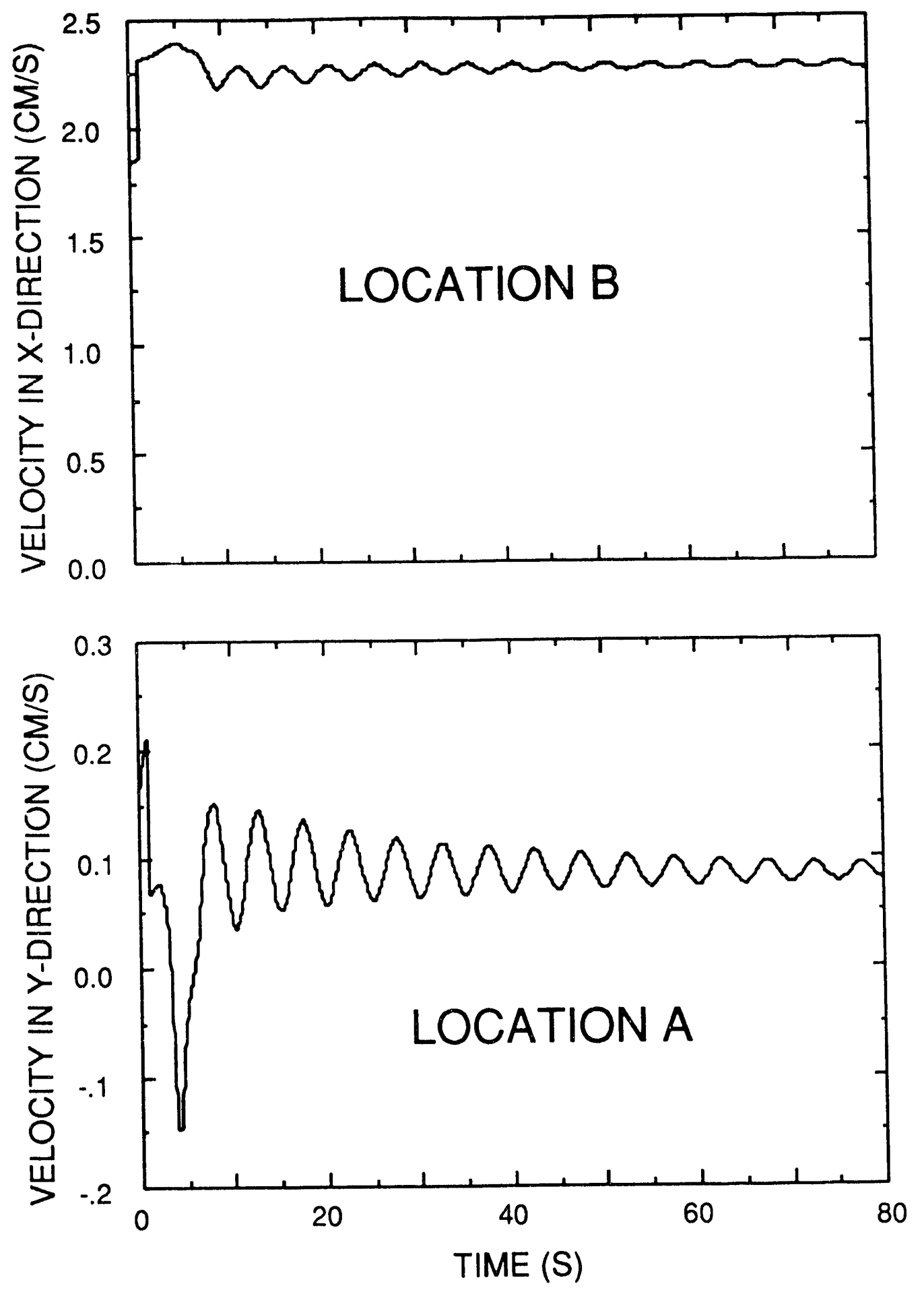

Fig. 6. Time-history plots of the normal and streamwise velocity components at two locations (see Fig. 4) for flow around a rectangular block, $\operatorname{Re}=30$, showing the tendency toward steady flow. 


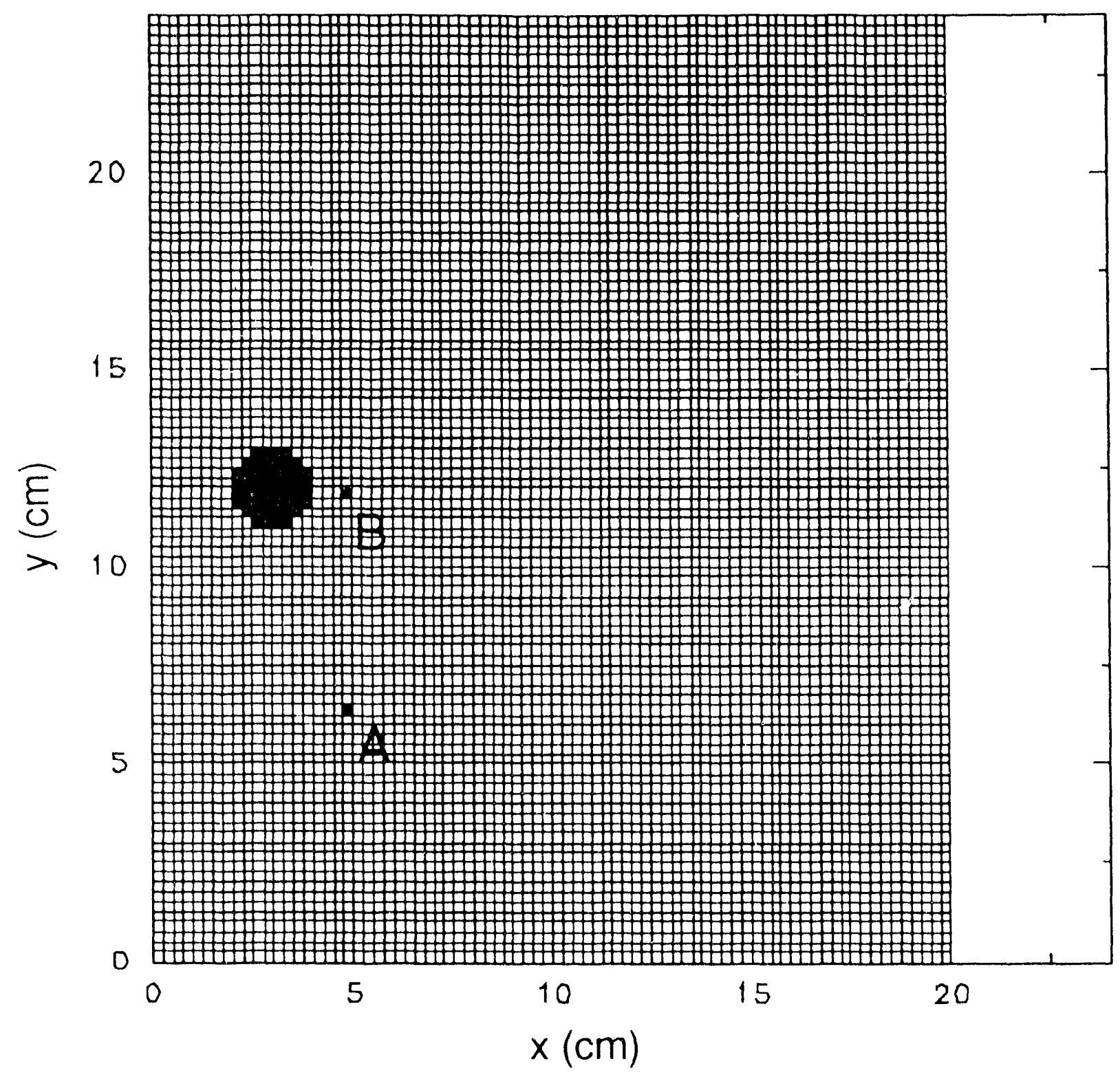

Fig. 7. Computational mesh (uniform) for the flow past circular cylinder problem. The number of cells in $x$ and $y$ are $80 \times 96$. Each cell is $0.25 \times 0.25 \mathrm{~cm}$. Points $A$ and $B$ indicate locations for velocity time-history plots in Figs. 9 and 10. 


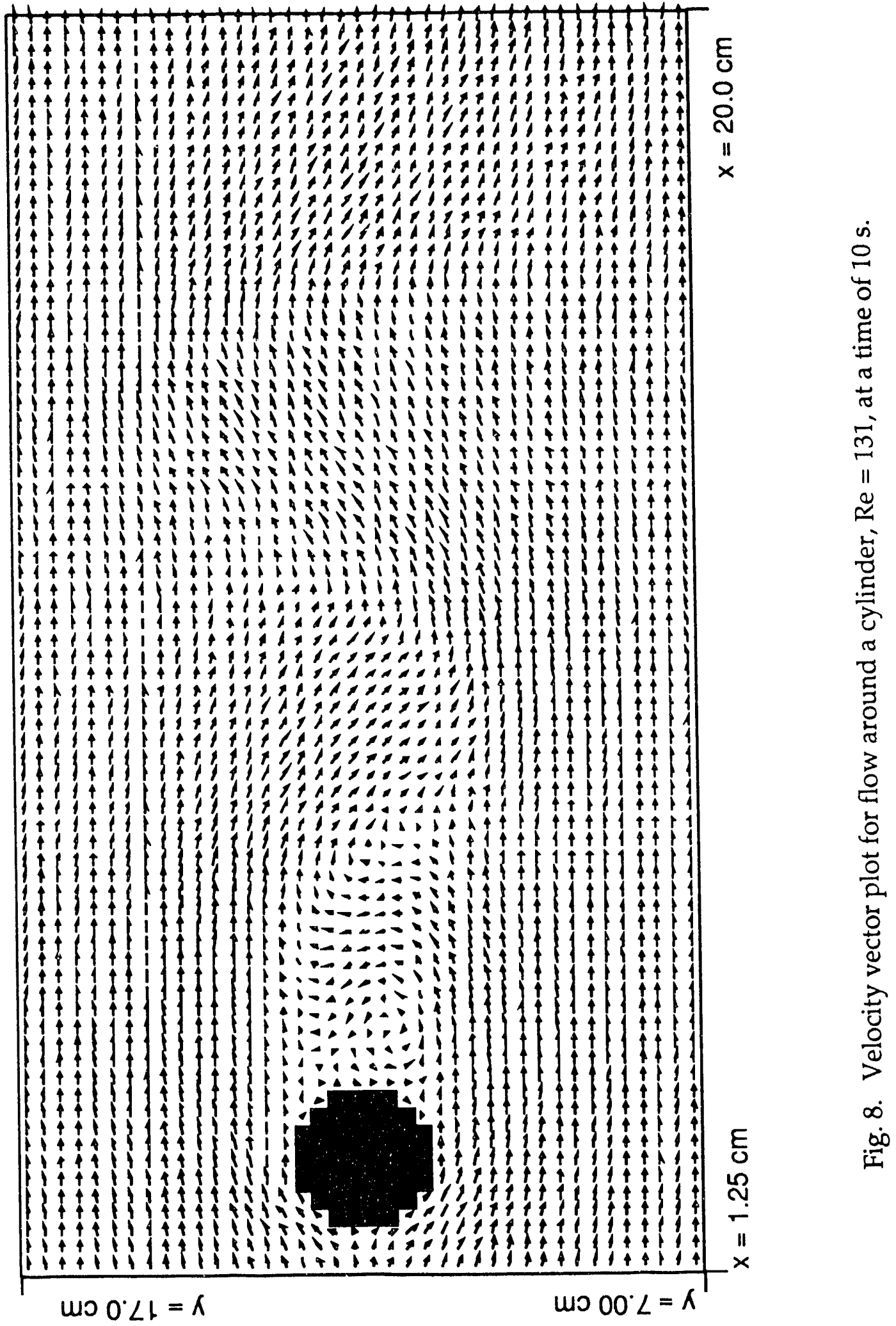


DRAFT
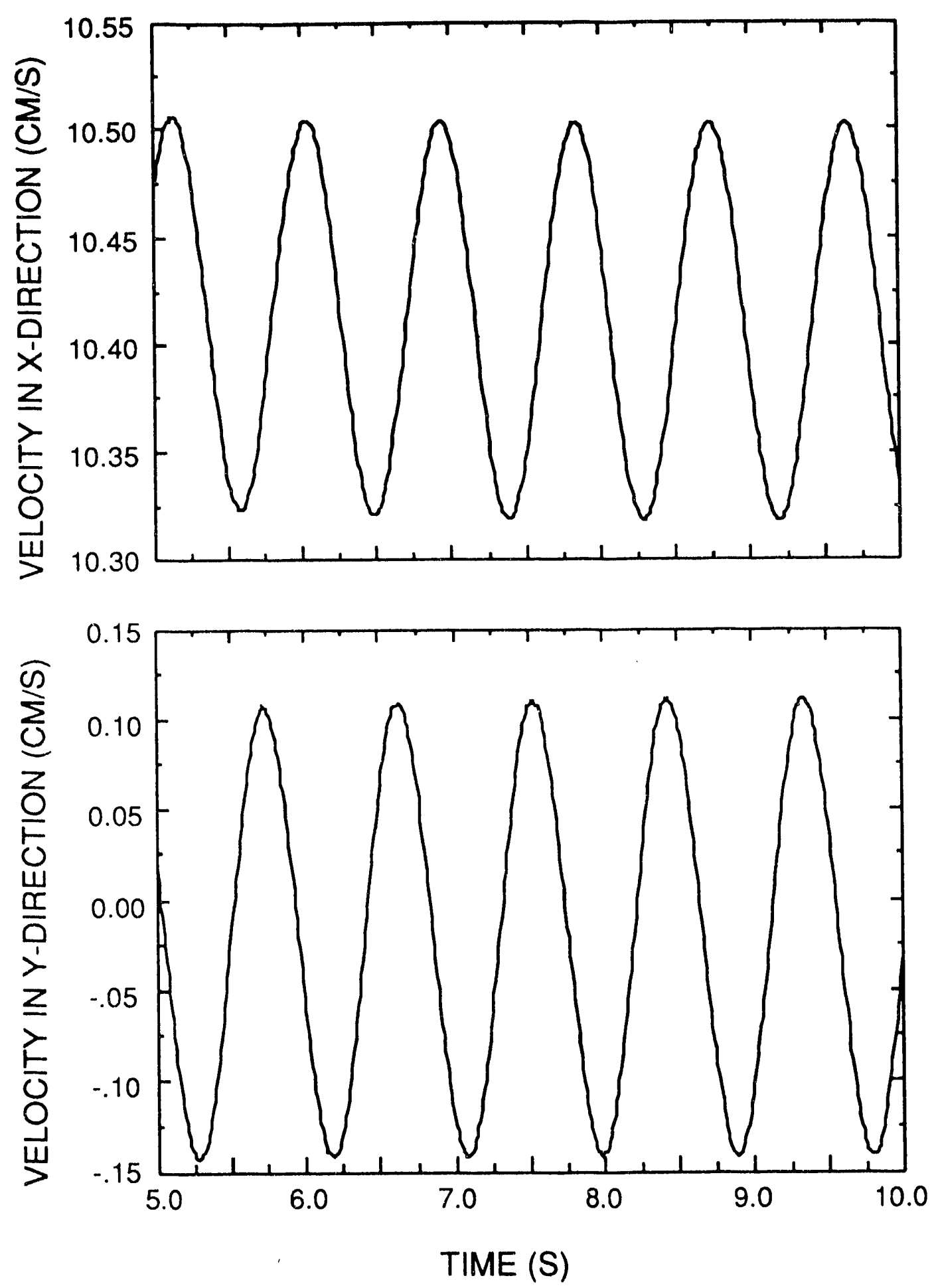

Fig. 9. Time-history plots of the normal and streamwise velocity components for flow over a cylinder, $\operatorname{Re}=131$. The location is at half a diameter behind and 3 diameters below the center of the cy!inder (Point A in Fig. 7). 
DRAFT
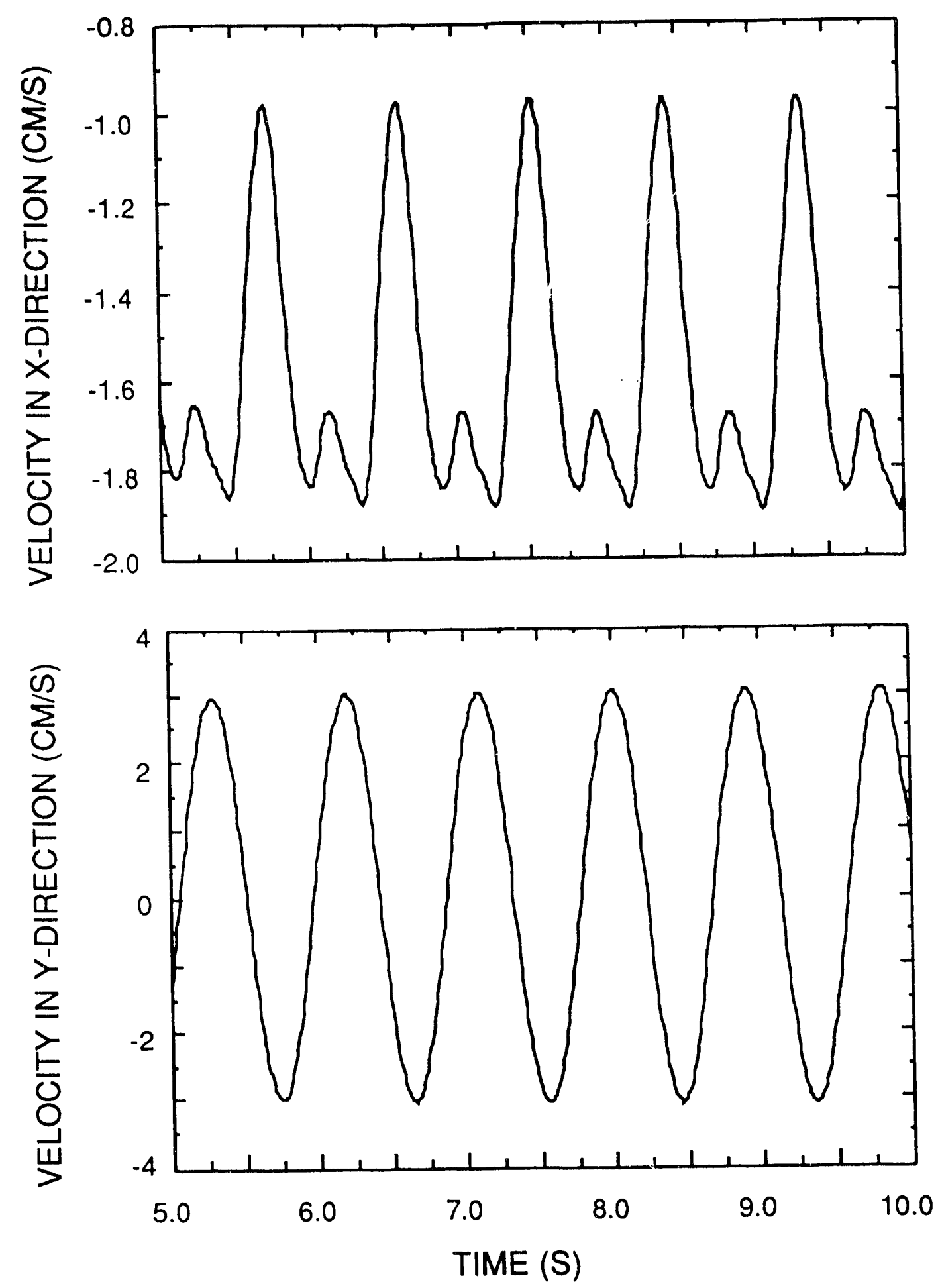

Fig. 10. Time-history plots of the normal and streamwise velocity components for flow over a cylinder, $\operatorname{Re}=131$. The location is at half a diameter behind and one cell (1/8 diameter) below the center of the cylinder (Point $B$ in Fig. 7). 


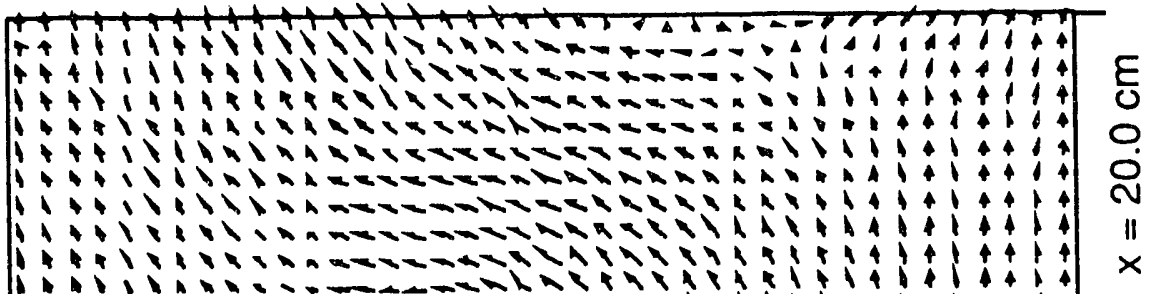

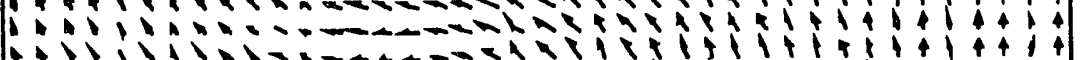

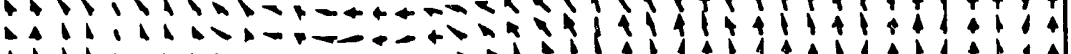

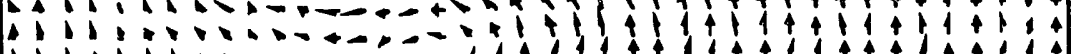

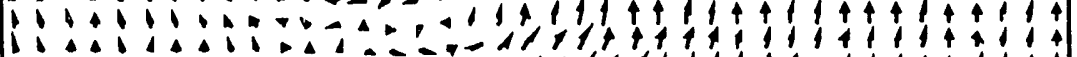

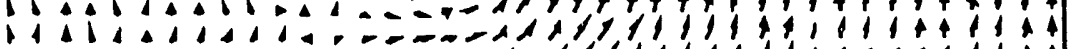

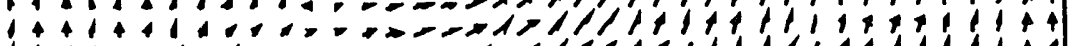

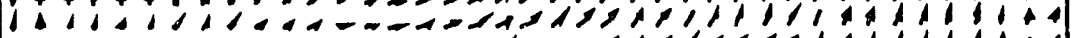

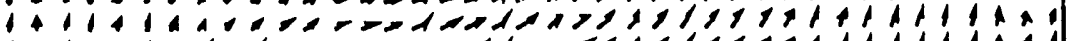

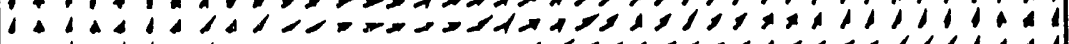

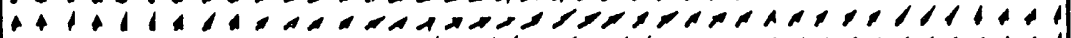

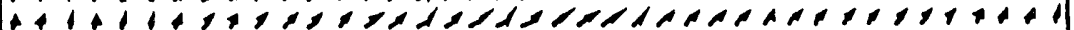

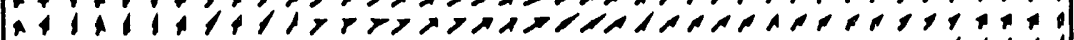

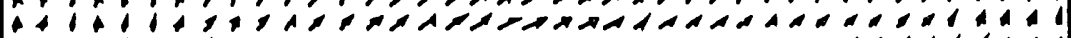

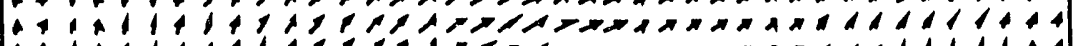

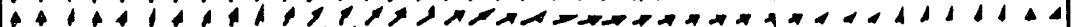

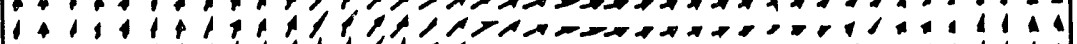

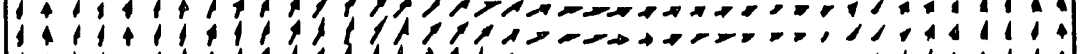

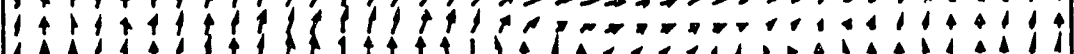

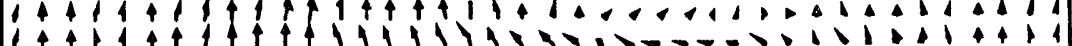

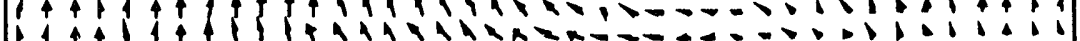

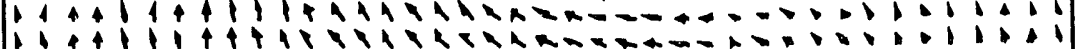

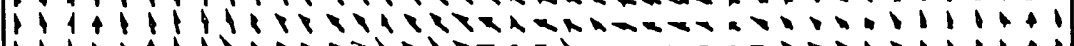

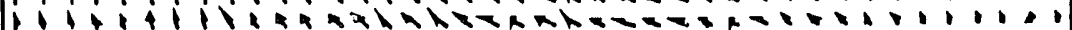

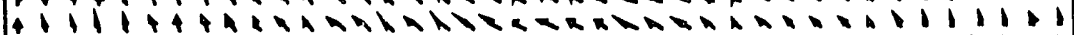

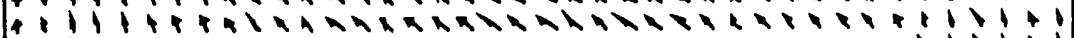

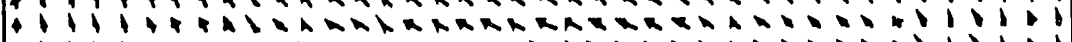

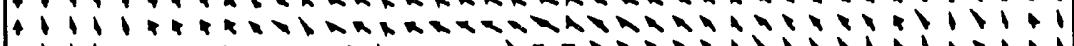

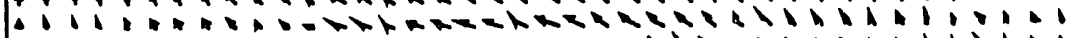
1 1. : : :- -

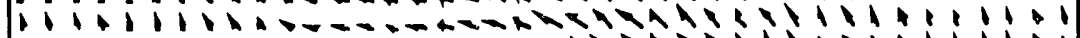

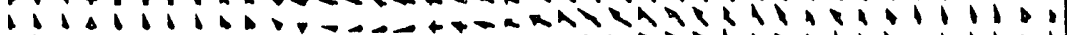

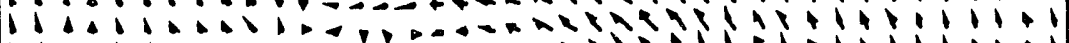

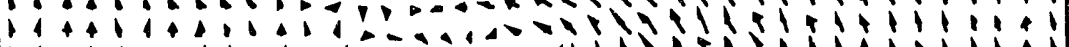

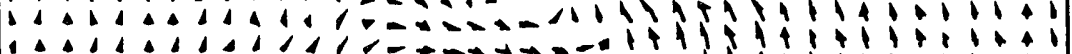

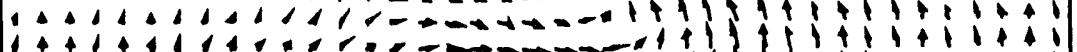

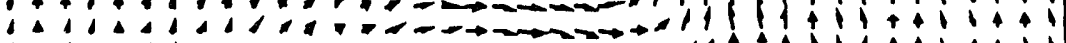

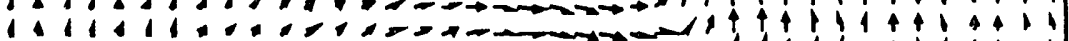

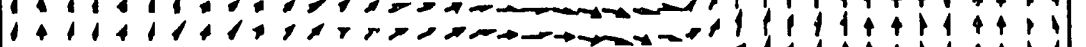

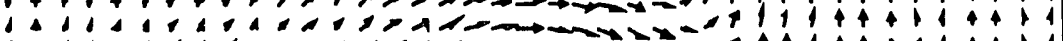

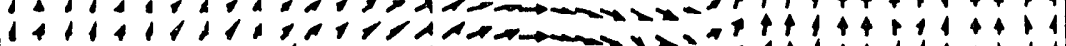

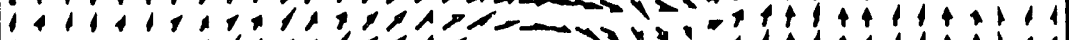

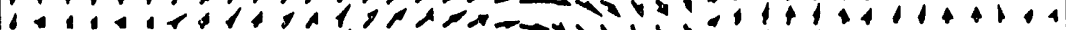

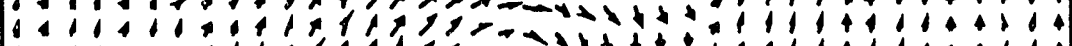

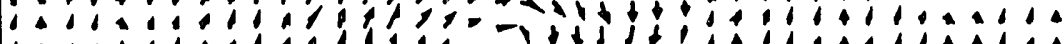

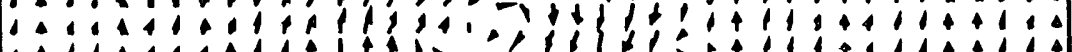
$1+19191+19\left\{\begin{array}{l}1 \\ 6\end{array}\right.$

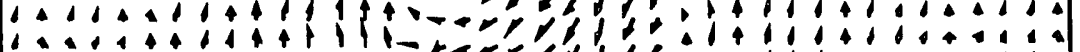

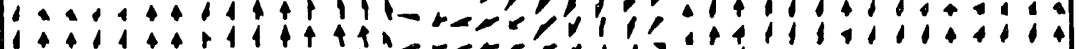

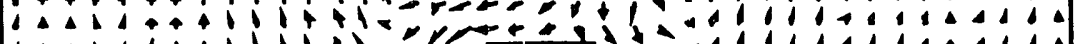

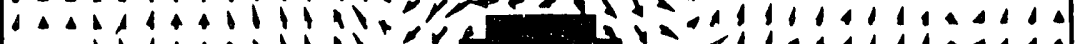

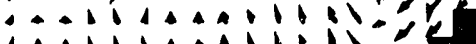

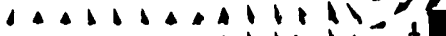

$1411:+411+11=$

AABDADIVIN-

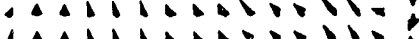

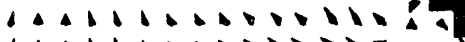

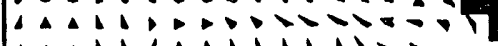

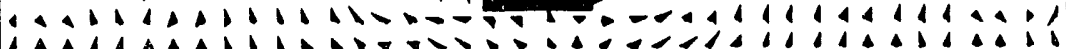

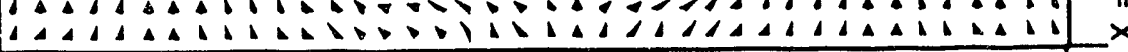

wo $0^{\circ} \angle L=\Lambda \quad$ wo $00^{\circ} L=\Lambda^{\prime}$ $1=1$
1

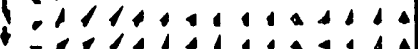

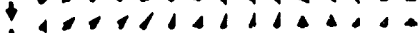

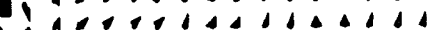



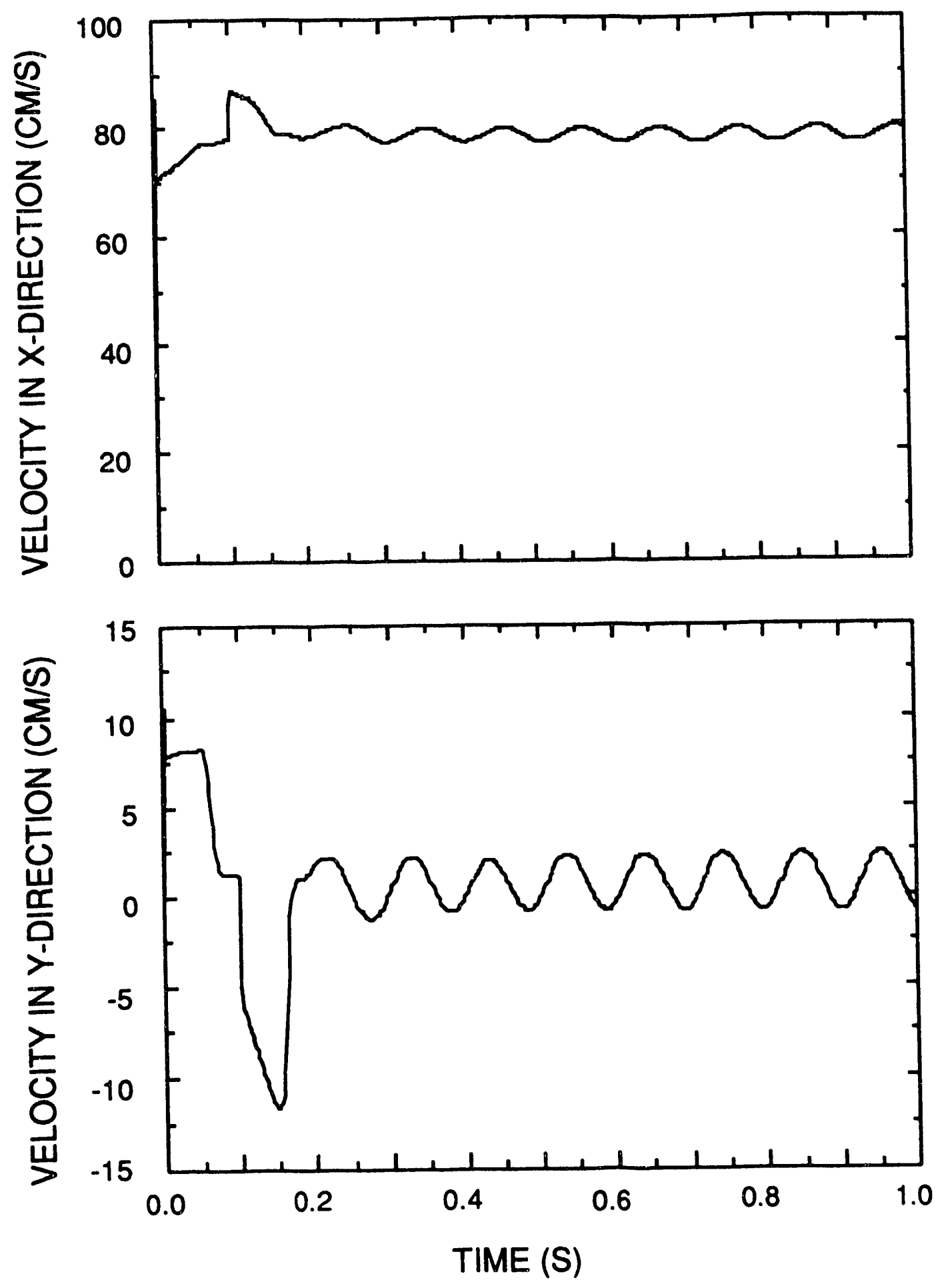

Fig. 12. Time-history plots of the normal and streamwise velocity components for flow over a cylinder, $\mathrm{Re}=1000$. The location is at half-a-diameter behind and 6 diameters below the center of the cylinder. 


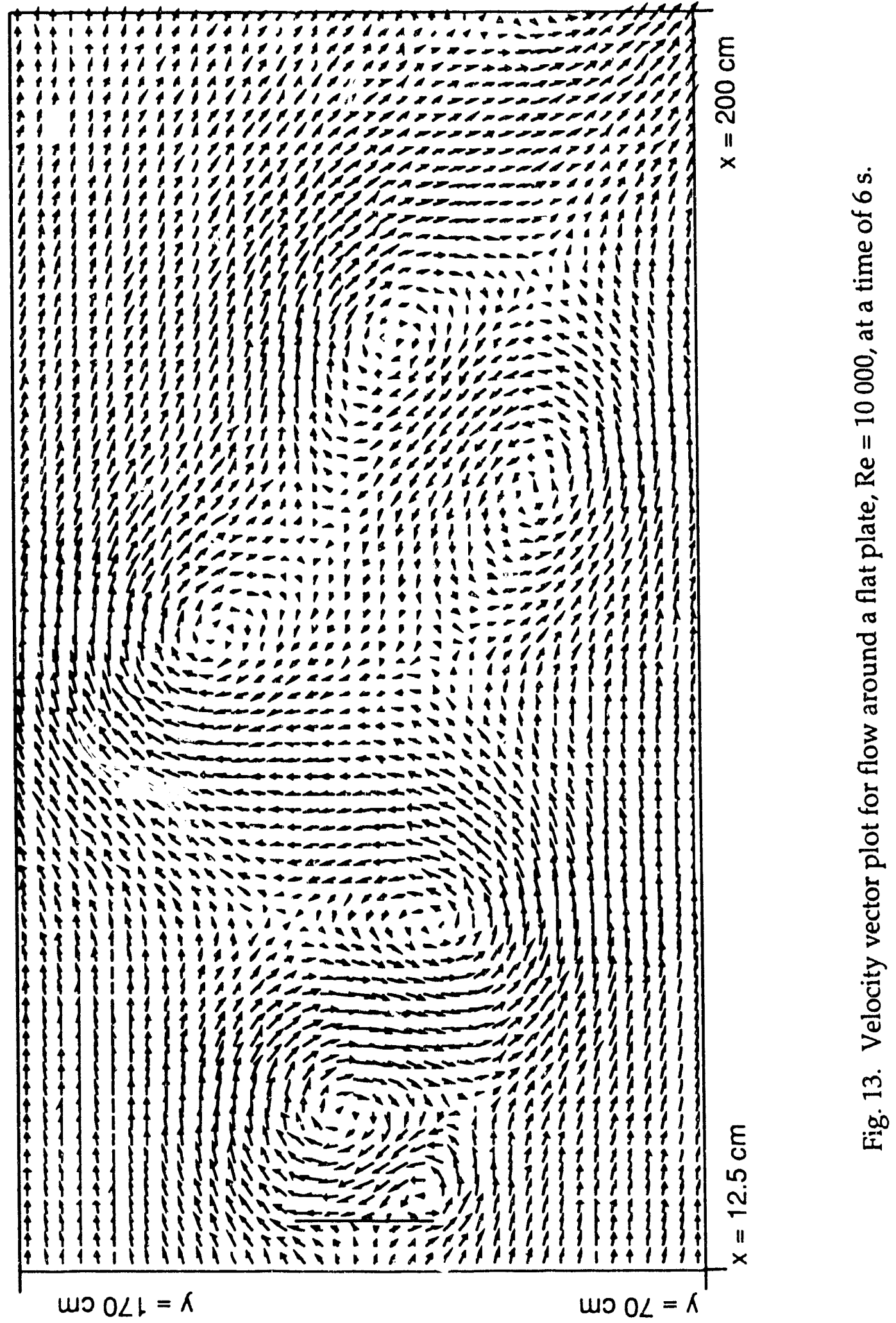



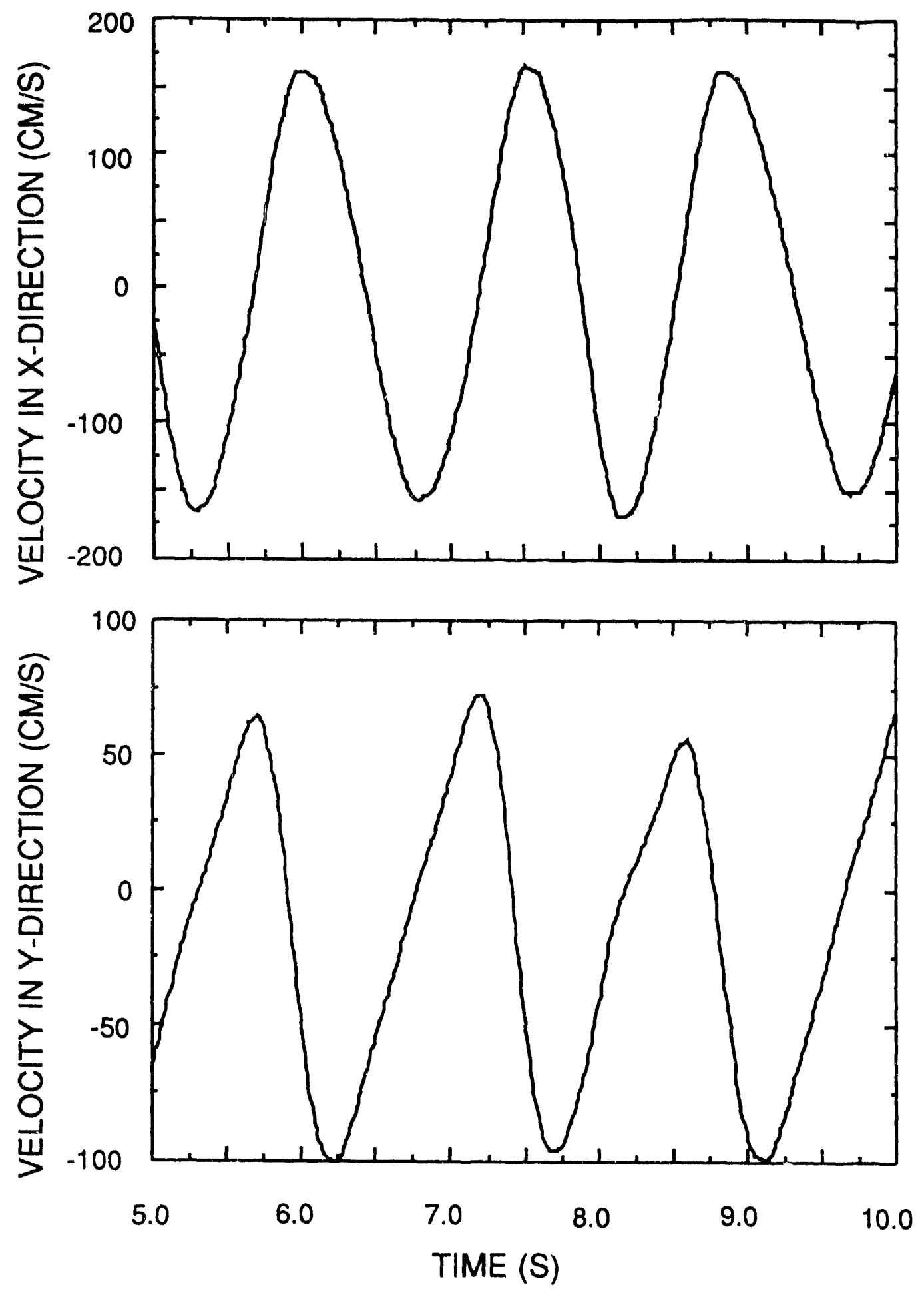

Fig. 14. Time-history plots of the normal velocity component at two locations for flow around a flat plate, $\operatorname{Re}=10000$. Top: $20 \mathrm{~cm}$ (1 plate height) behind and $2.5 \mathrm{~cm}$ (1/8 plate height) below centerline of the plate. Bottom: $60 \mathrm{~cm}$ ( 3 plate heights) and $25 \mathrm{~cm}$ (1.25 plate height) below centerline of the plate. 

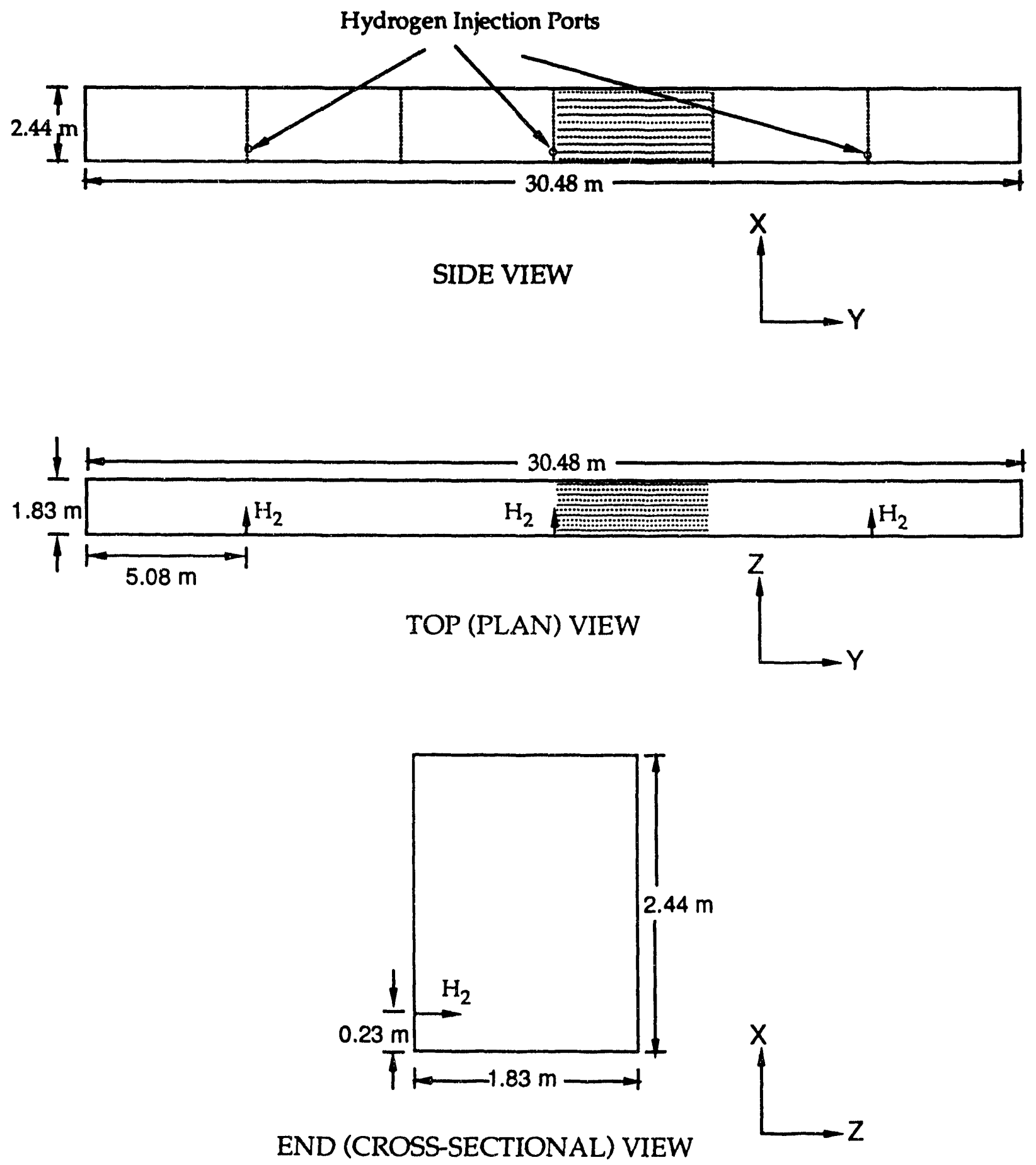

Fig. 15. Schematic diagram of the Sandia FLAME channel showing locations of hydrogen injection. The shaded area indicates the symmetrical region modeled in the computation. 


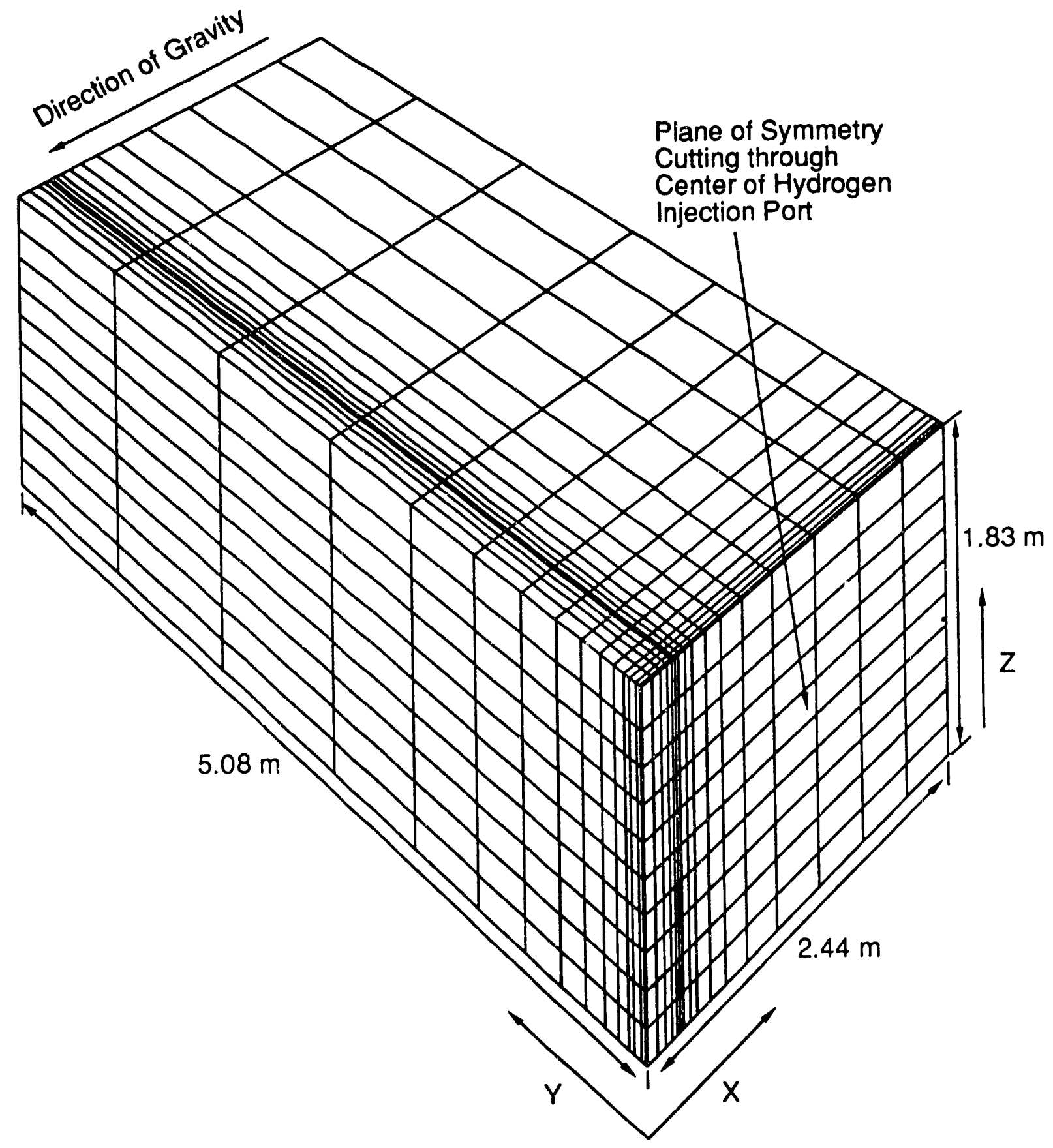

Fig. 16. Computational domain for the Sandia FLAME simulation calculation showing the variable mesh. 


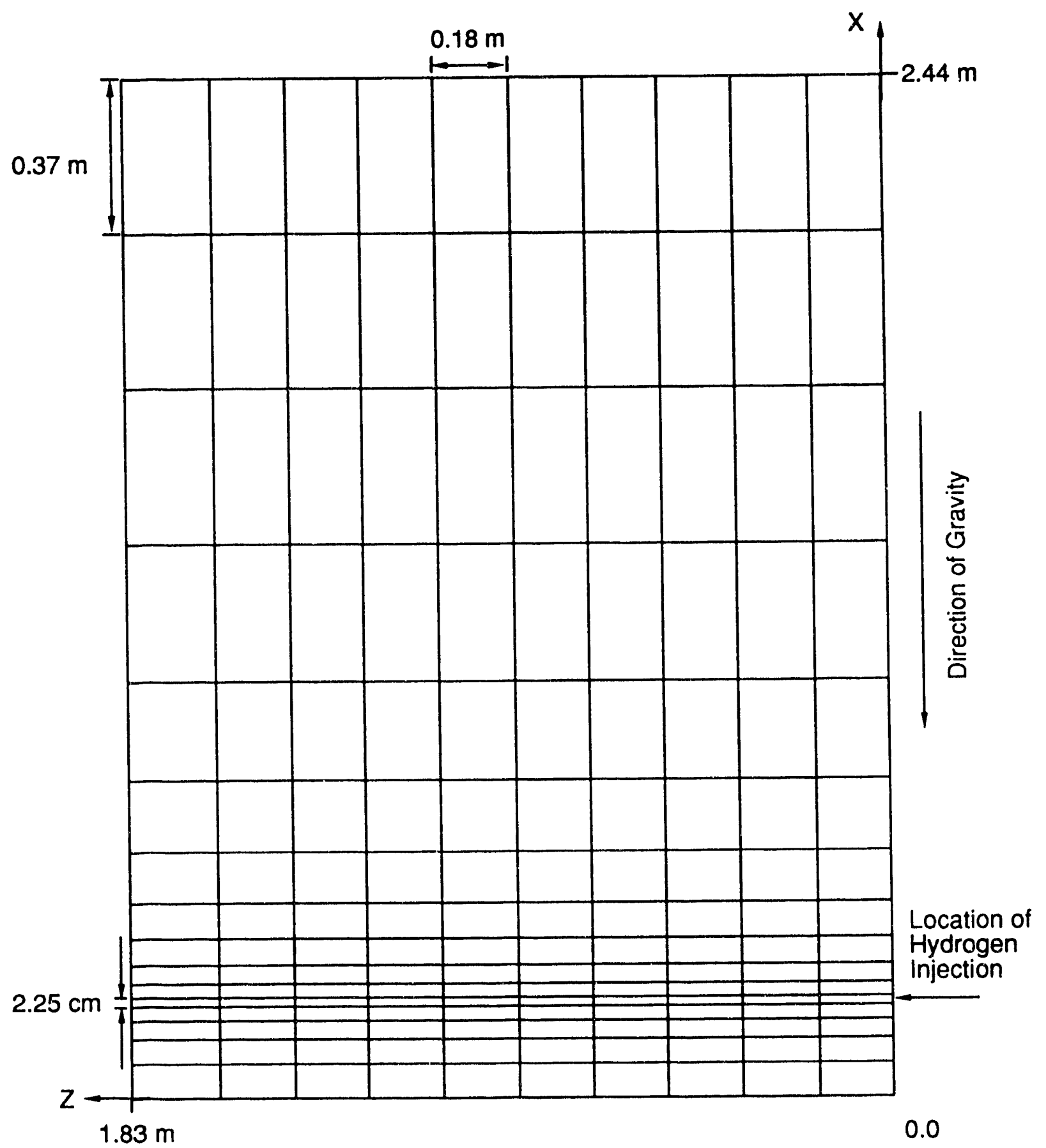

Fig. 17. Two-dimensional view of the computational mesh used for the Sandia FLAME calculation showing the location where hydrogen is injected. 


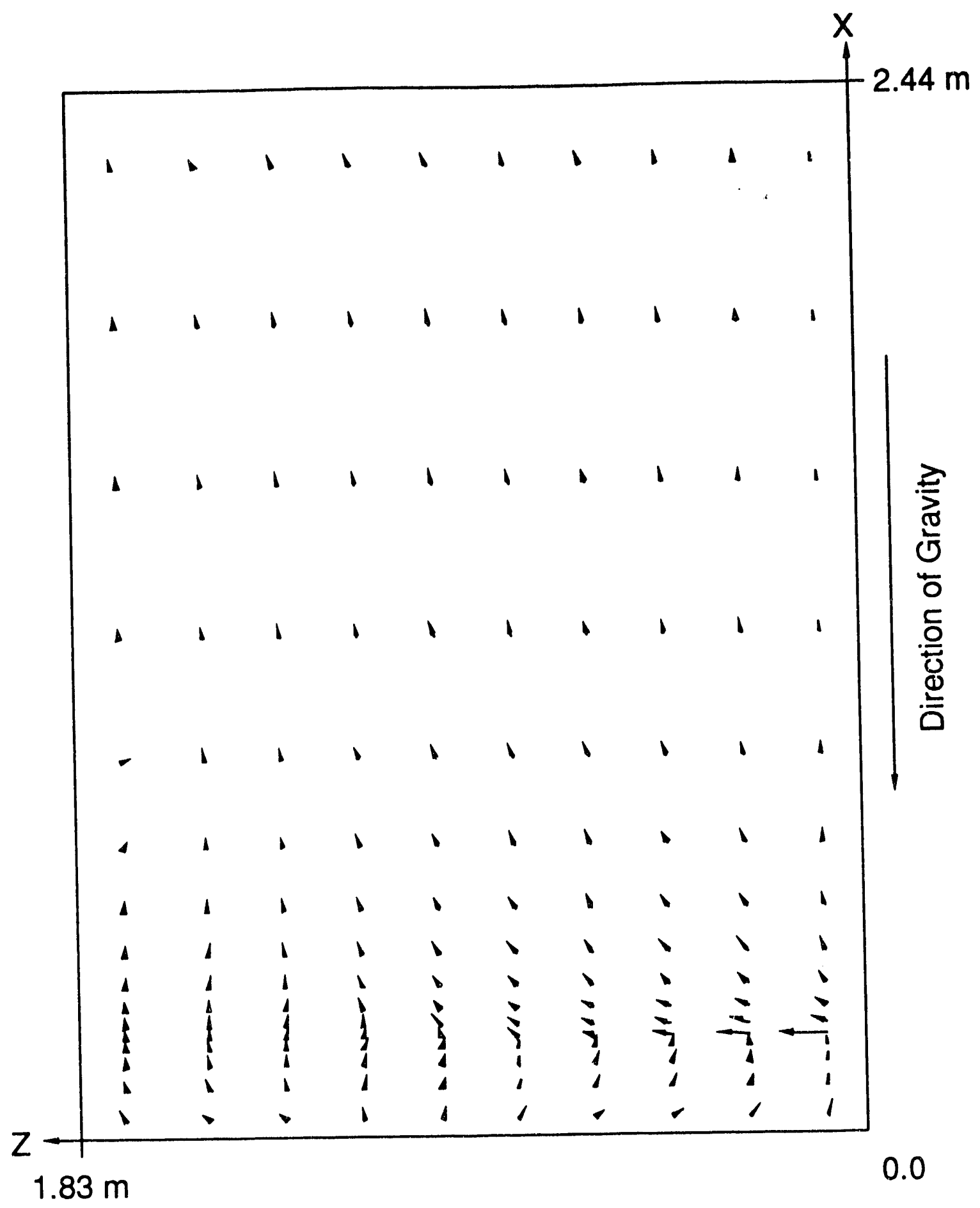

Fig. 18. Plot of the calculated velocity vectors in the Sandia FLAME experiment at the plane of injection at $100 \mathrm{~s}$ after initiation of the hydrogen injection. The maximum velocity magnitude (averaged at cell centers) is $22.3 \mathrm{~m} / \mathrm{s}$. 


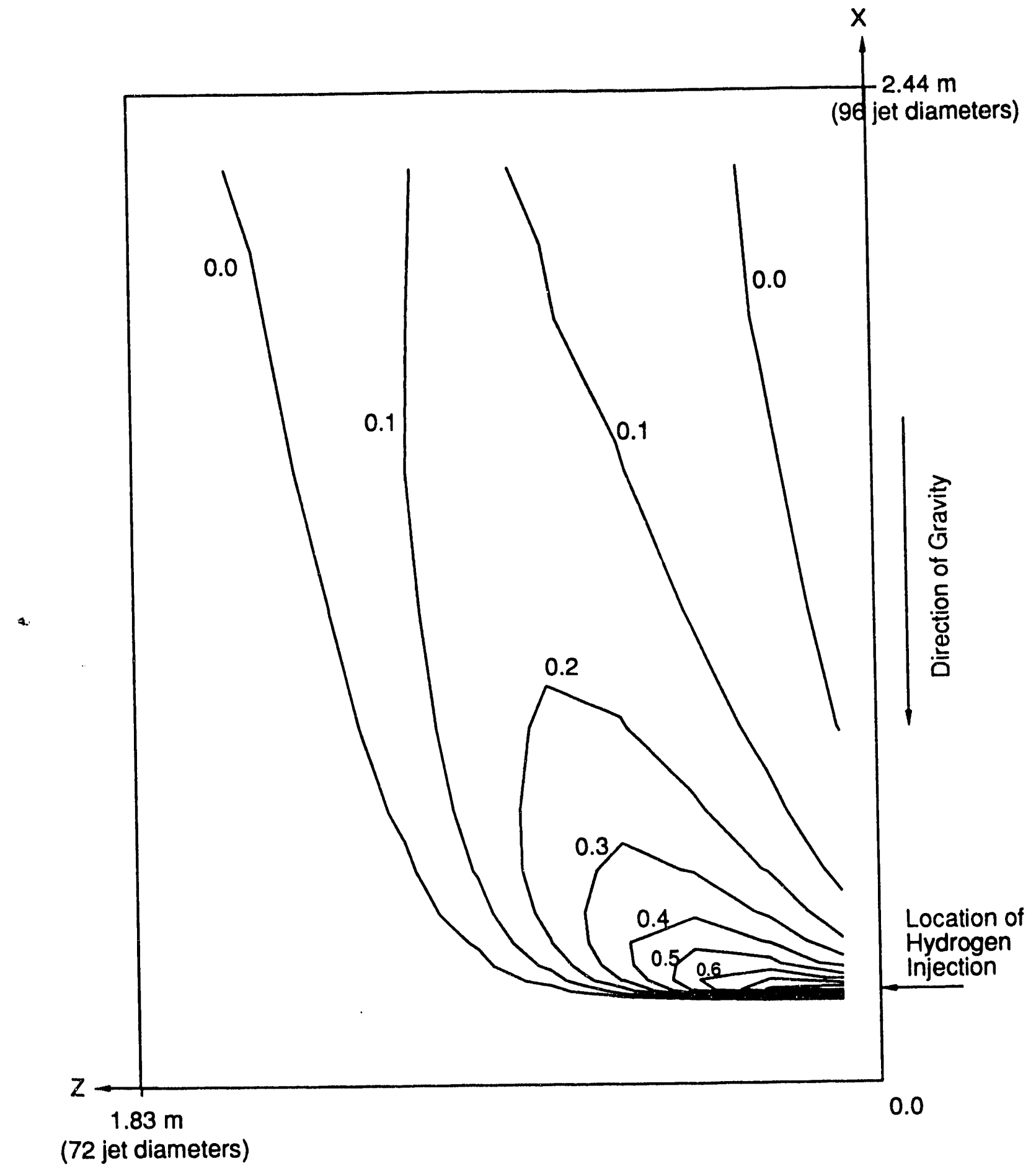

Fig. 19. Contour plot of the calculated hydrogen volume fraction in the Sandia FLAME experiment at the plane of injection at $100 \mathrm{~s}$ after the hydrogen injection begins, showing development of the buoyant plume. 


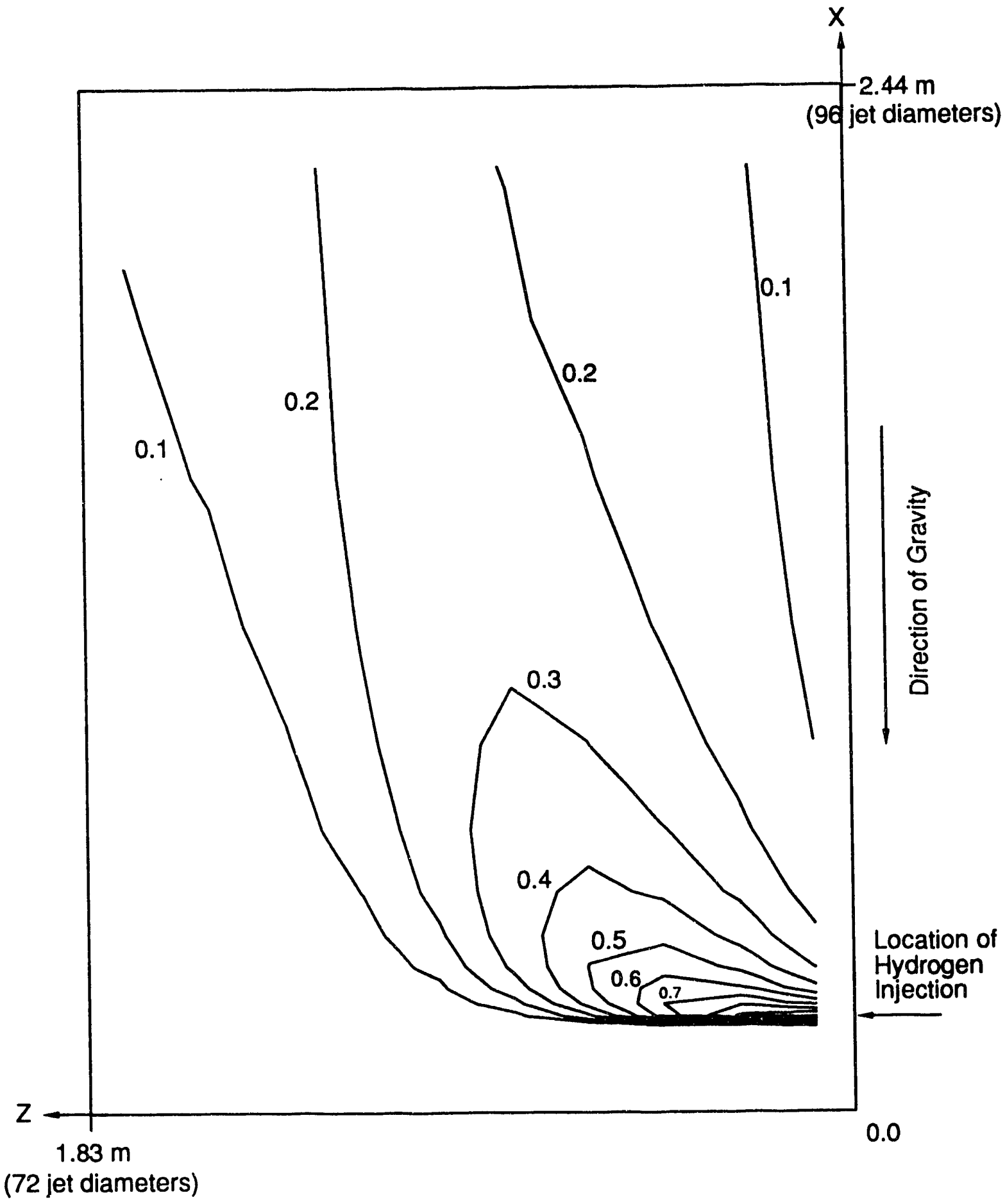

Fig. 20. Contour plot of the calculated hydrogen volume fraction in the Sandia FLAME experiment at the plane of injection at $200 \mathrm{~s}$ after the hydrogen injection begins. Note the gradual spreading of the buoyant plume. 


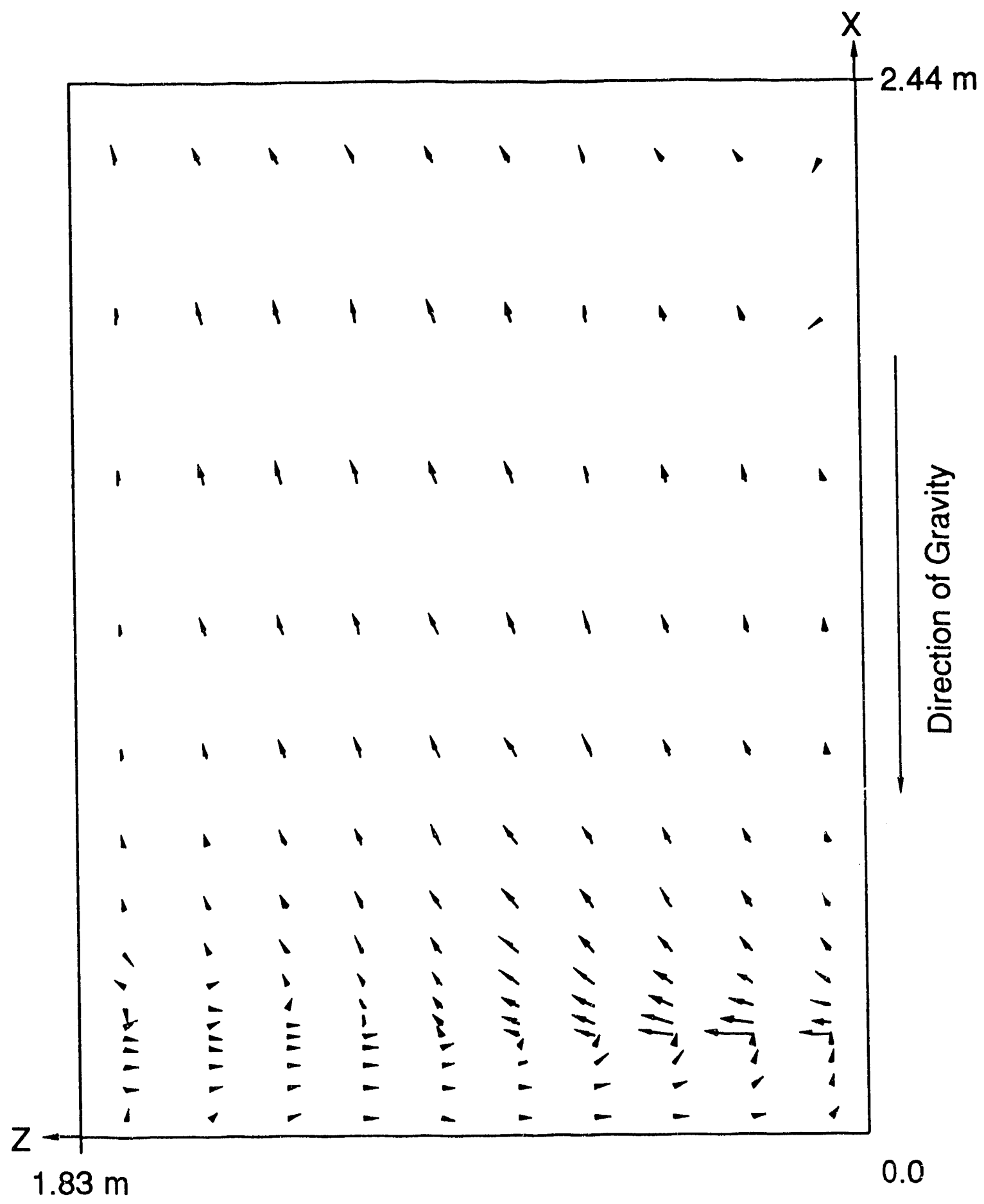

Fig. 21. Plot of the computed velocity vectors in the Sandia FLAME experiment at the plane of injection at $487 \mathrm{~s}$ or immediately after the injection stops.

The maximum velocity magnitude (averaged at cell centers) is $7.2 \mathrm{~m} / \mathrm{s}$. 


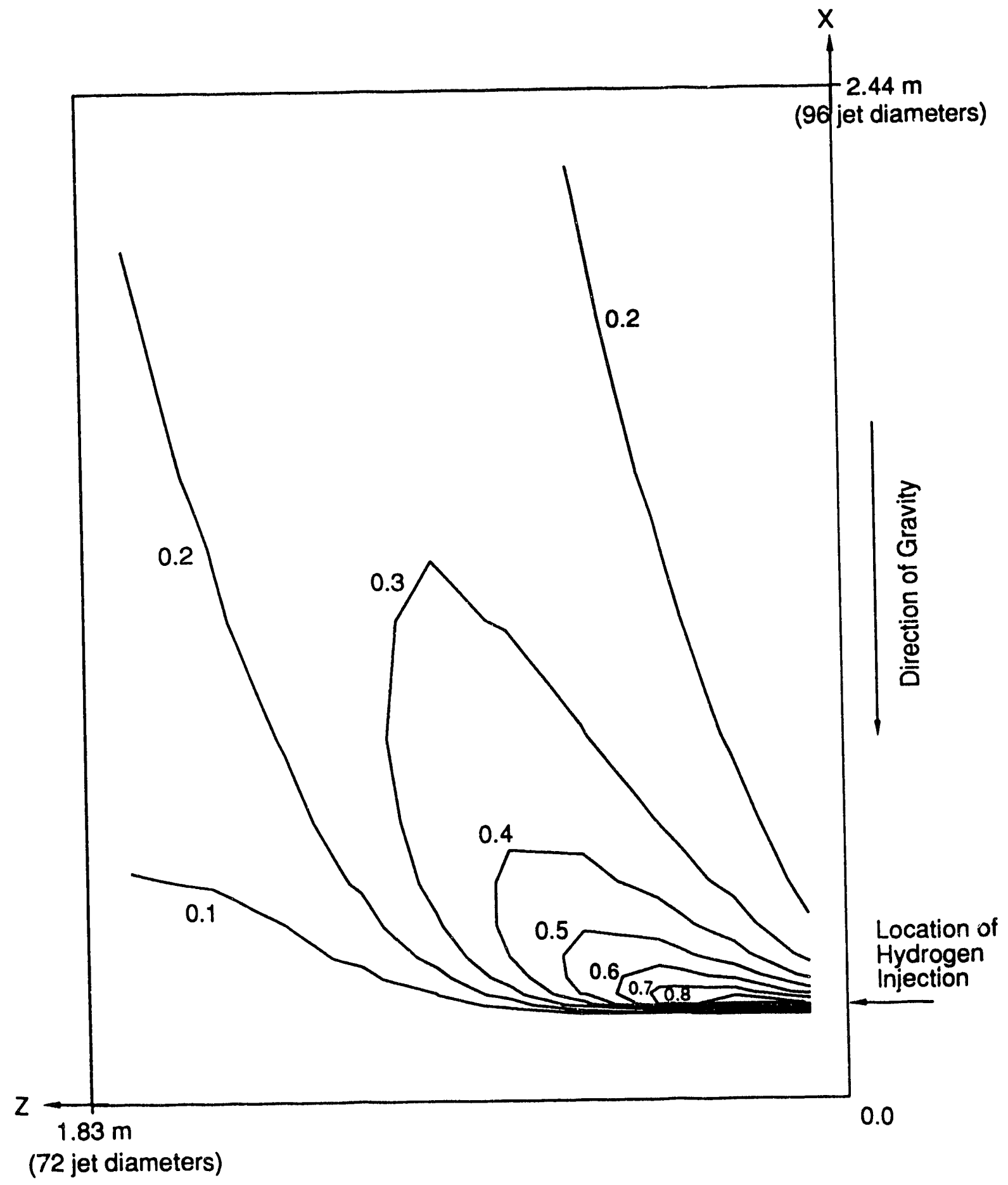

Fig. 22. Computed hydrogen volume fraction contours in the Sandia FLAME experiment at the plane of injection at $487 \mathrm{~s}$, immediately after the injection stops. 


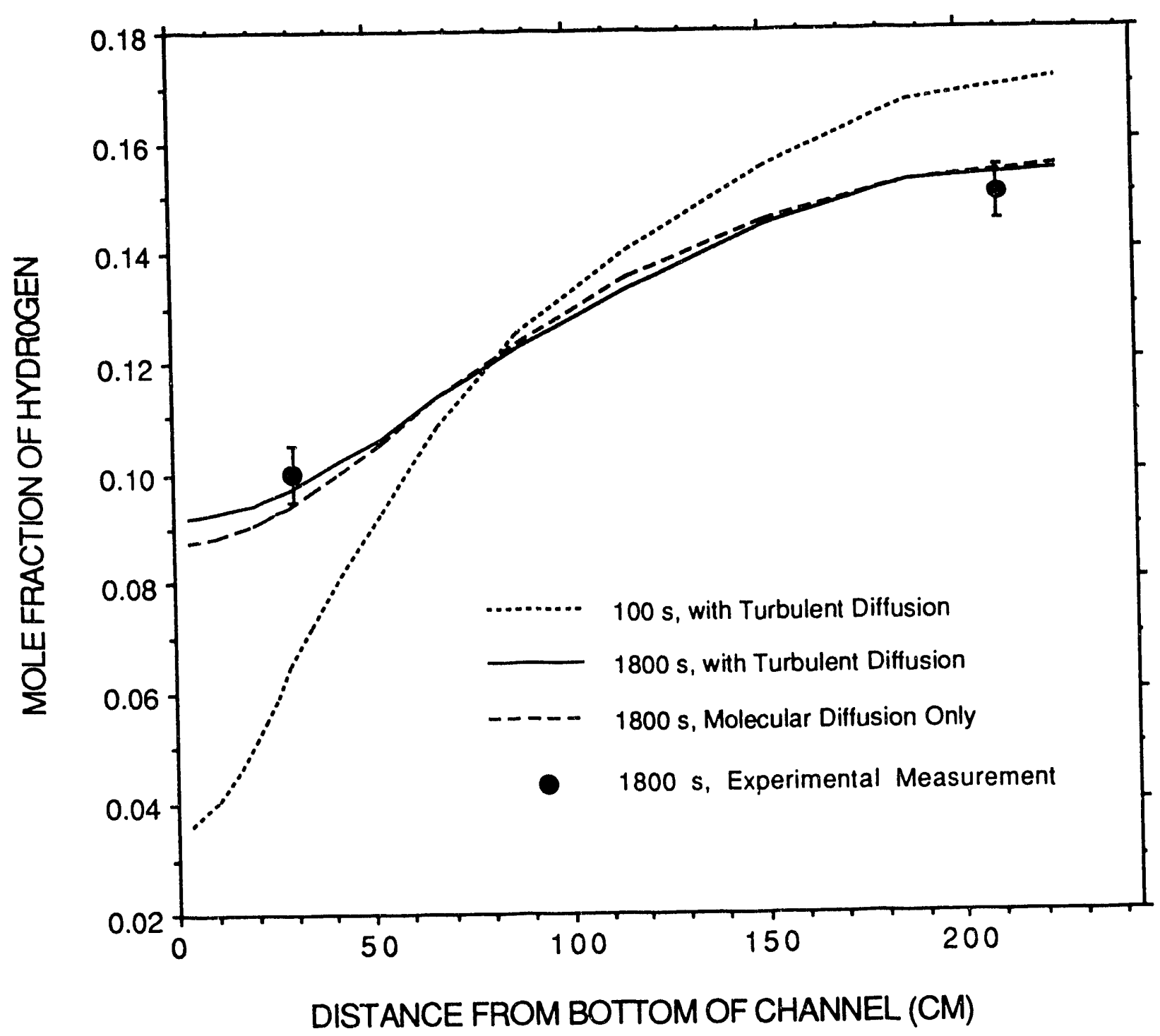

Fig. 23. Calculated profile of hydrogen mole fraction in the Sandia FLAME experiment as a function of distance from the bottom of the channel at $100 \mathrm{~s}$ and $1800 \mathrm{~s}$ after the injection stops. The two experimental data points (with error bars) at $1800 \mathrm{~s}$ are also shown. 

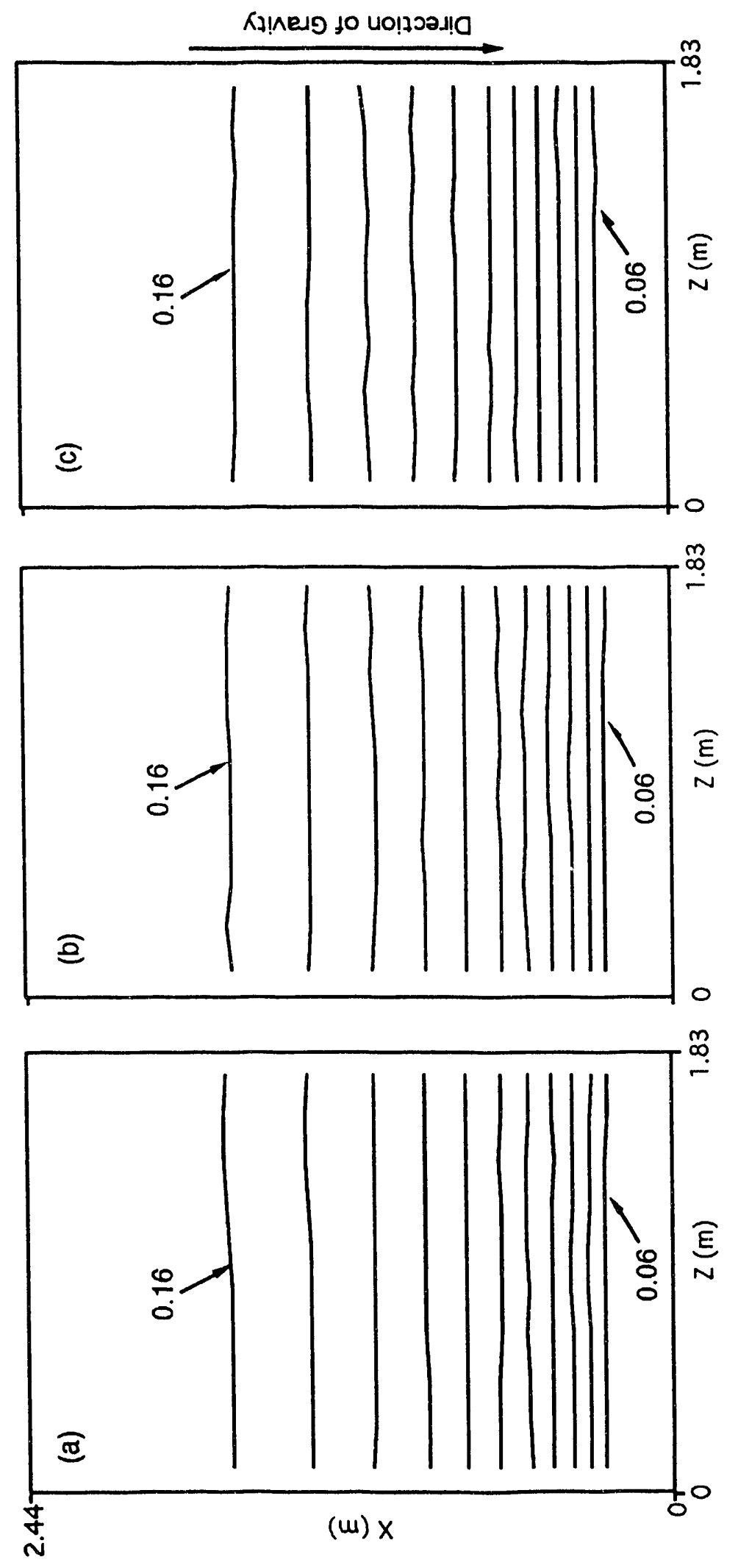

ธํํำ

急

壳

总

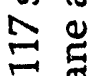

त $\overrightarrow{5} \cdot \dot{0}$

Ð 웜

몀

จํ.

$x \| \equiv$

즐

芒

> สี

$\Phi$ 잉

吉主

둥충

.

㐘的

응 웅

E己

ธำ

융형

을

苍壳 :

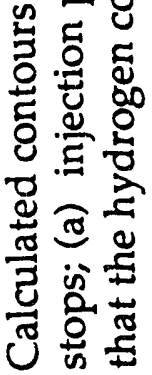

ป்

$\ddot{10}$ 


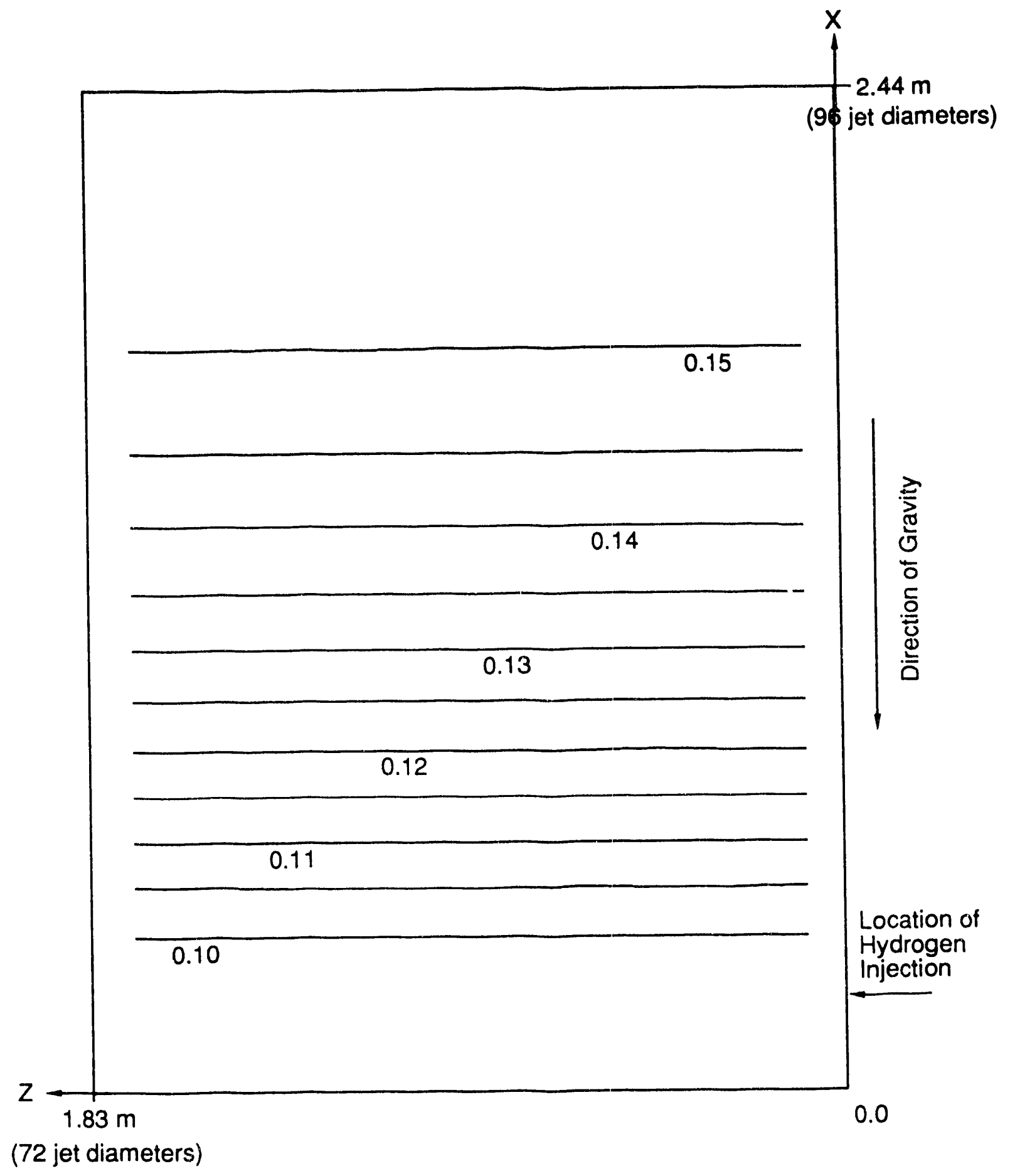

Fig. 25. Calculated hydrogen volume fraction contours in the Sandia FLAME experiment at the plane of injection $1800 \mathrm{~s}$ after the injection stops exhibiting vertical stratification of the hydrogen. 


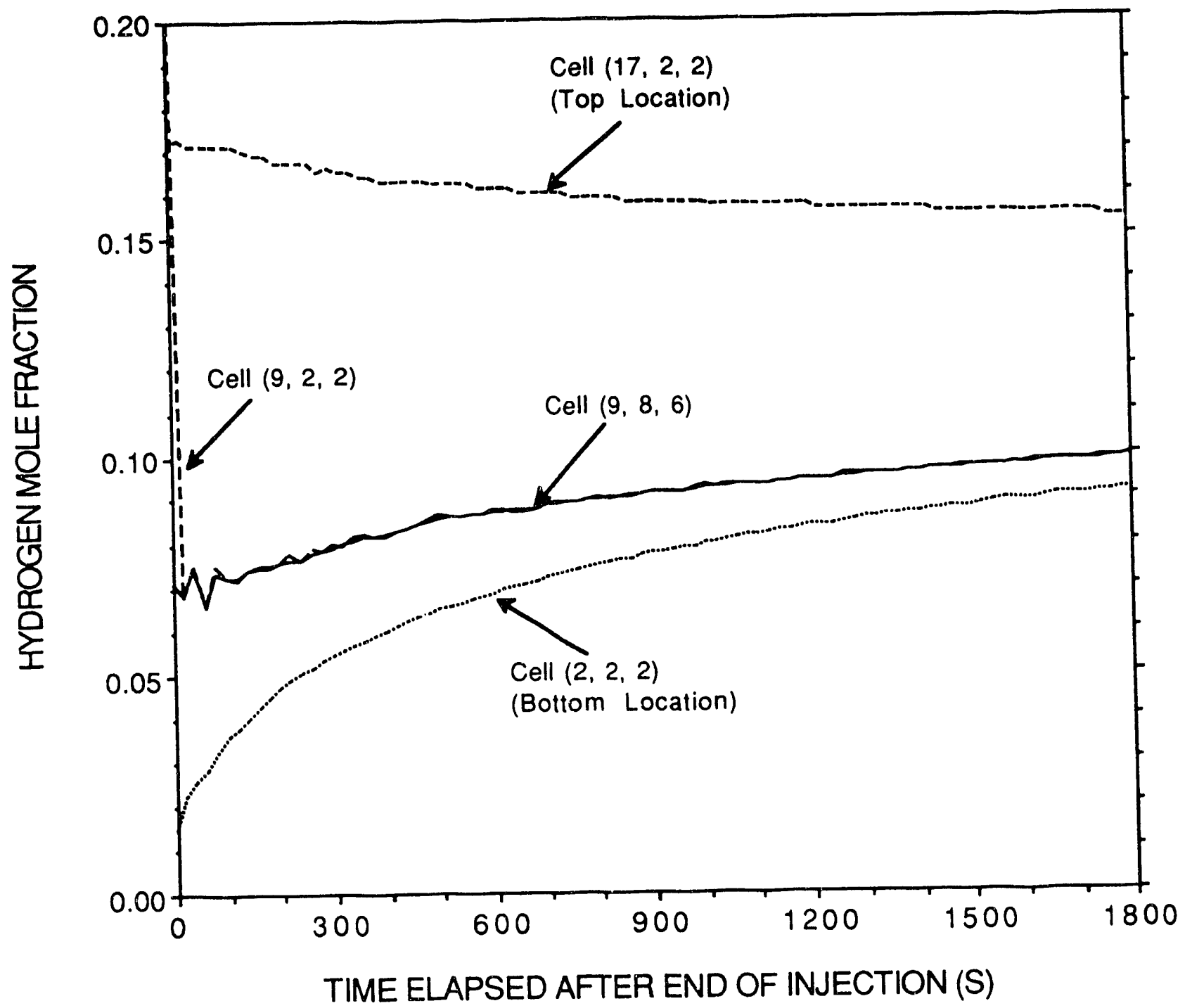

Fig. 26. Time-history plots of the calculated hydrogen mole fraction in the Sandia FLAME experiment at various locations. Note merging of the curves for the two locations at the same height (i.e., same first cell index, 9). 

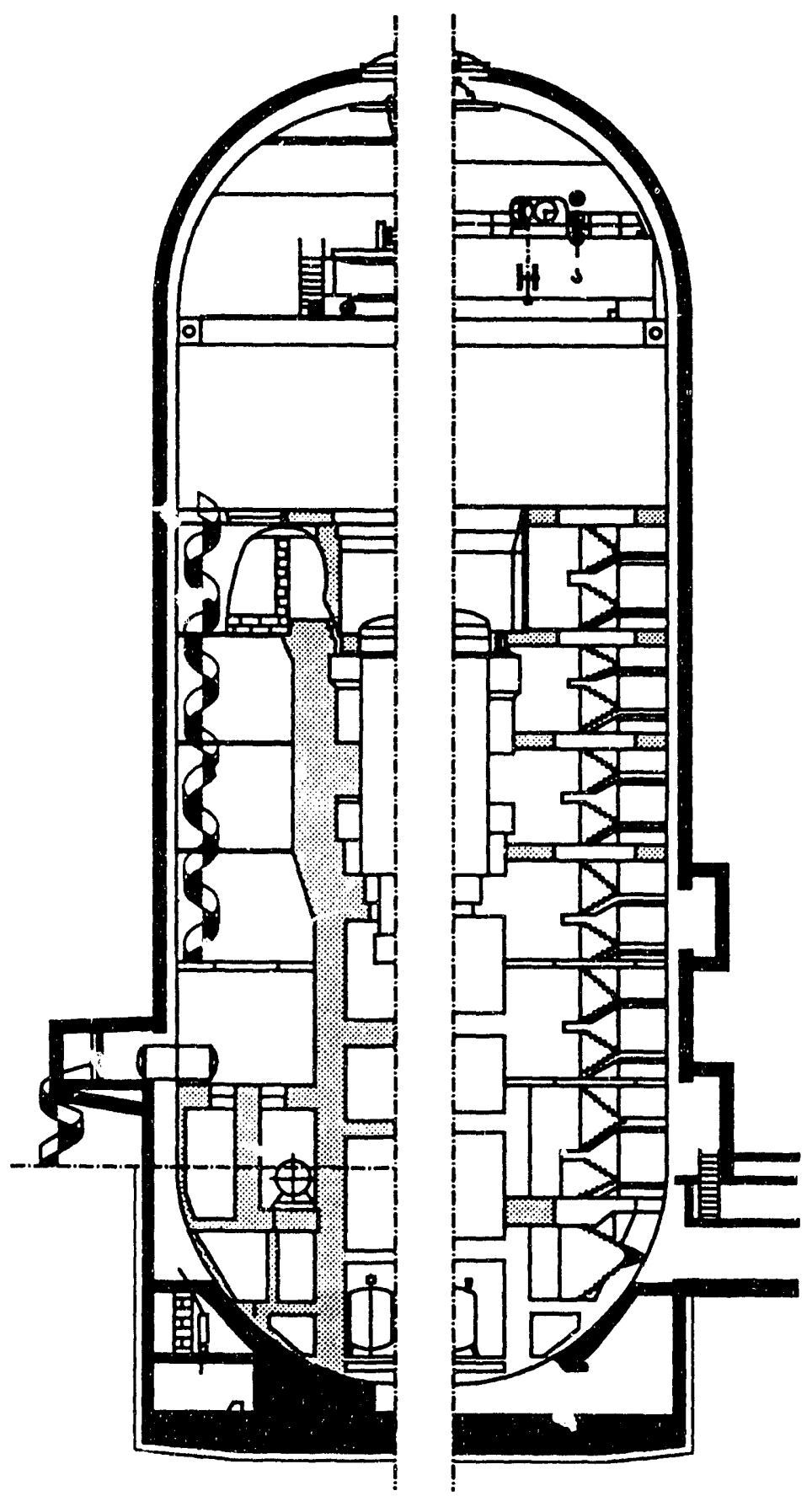

Fig. 27. Cross-sectional view of the HDR containment building at two angles. The cross section on the right shows the standard staircase; the spiral staircase is visible on the left. 


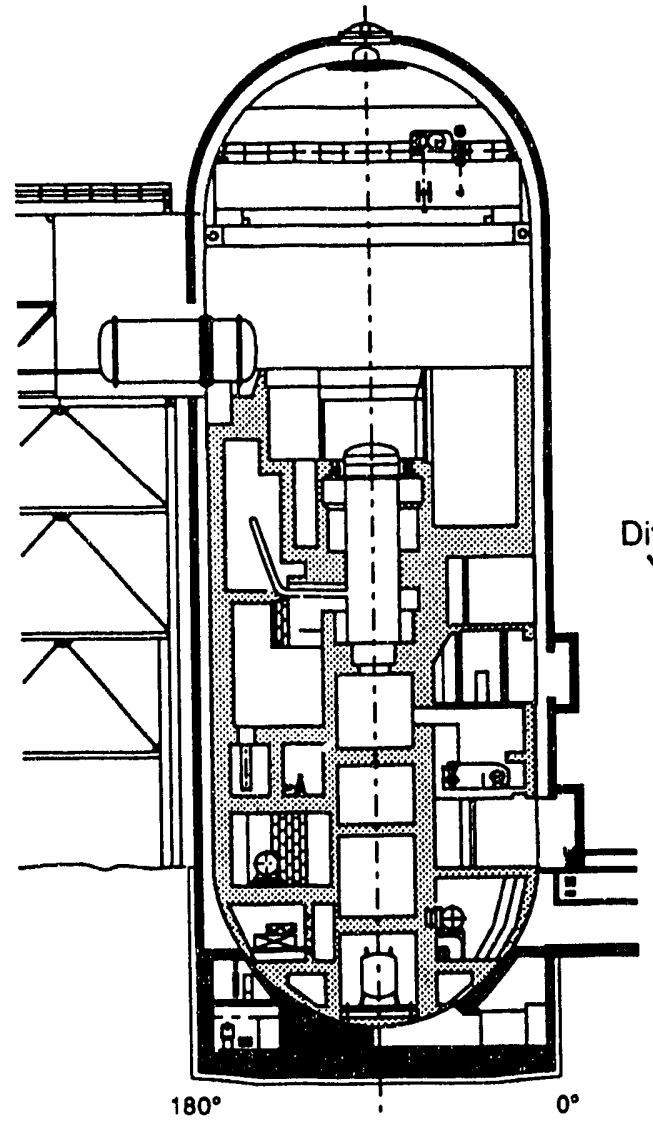

(a)

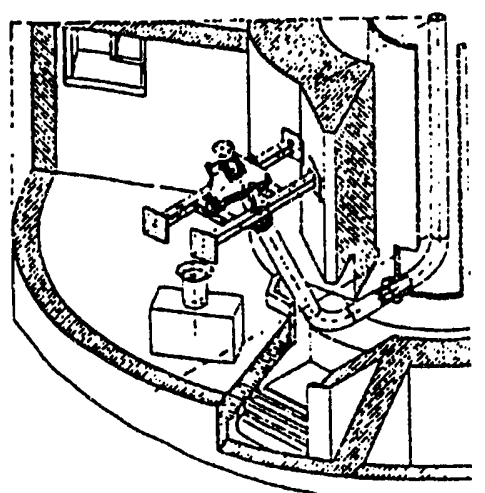

(b)

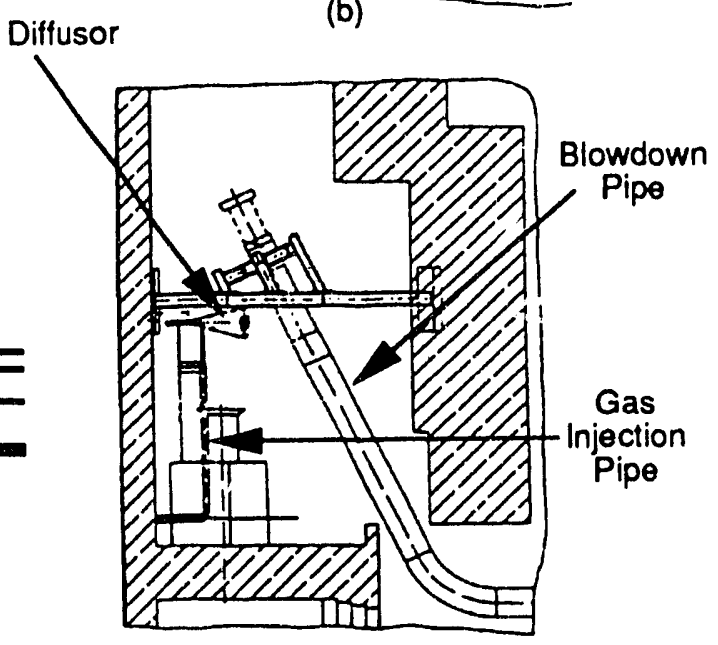

(c)

Fig. 28. (a) Cross-sectional view of the HDR containment facility showing the blowdown room. The blowdown pipe can be seen in the middle, extending from the lefi: side of the pressure vessel; (b) Perspective drawing of the blowdown room; (c) Details of the blowdown pipe and gas injection pipe and diffusor. 

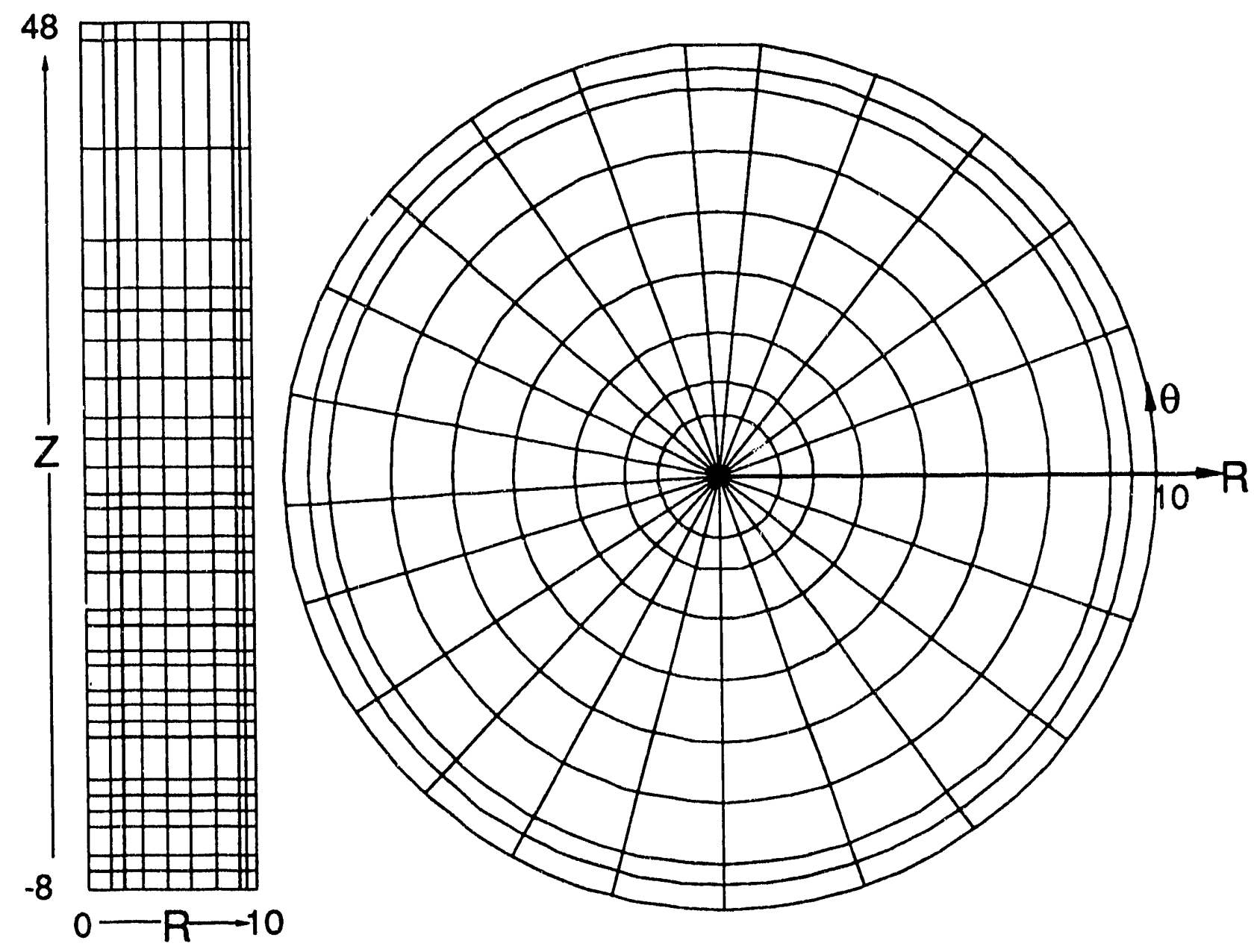

Fig. 29. Vertical (R-Z) and horizontal (R- $\theta)$ cut of the 3D mesh used for the HDR calculation. All dimensions are in $\mathrm{m}$. 


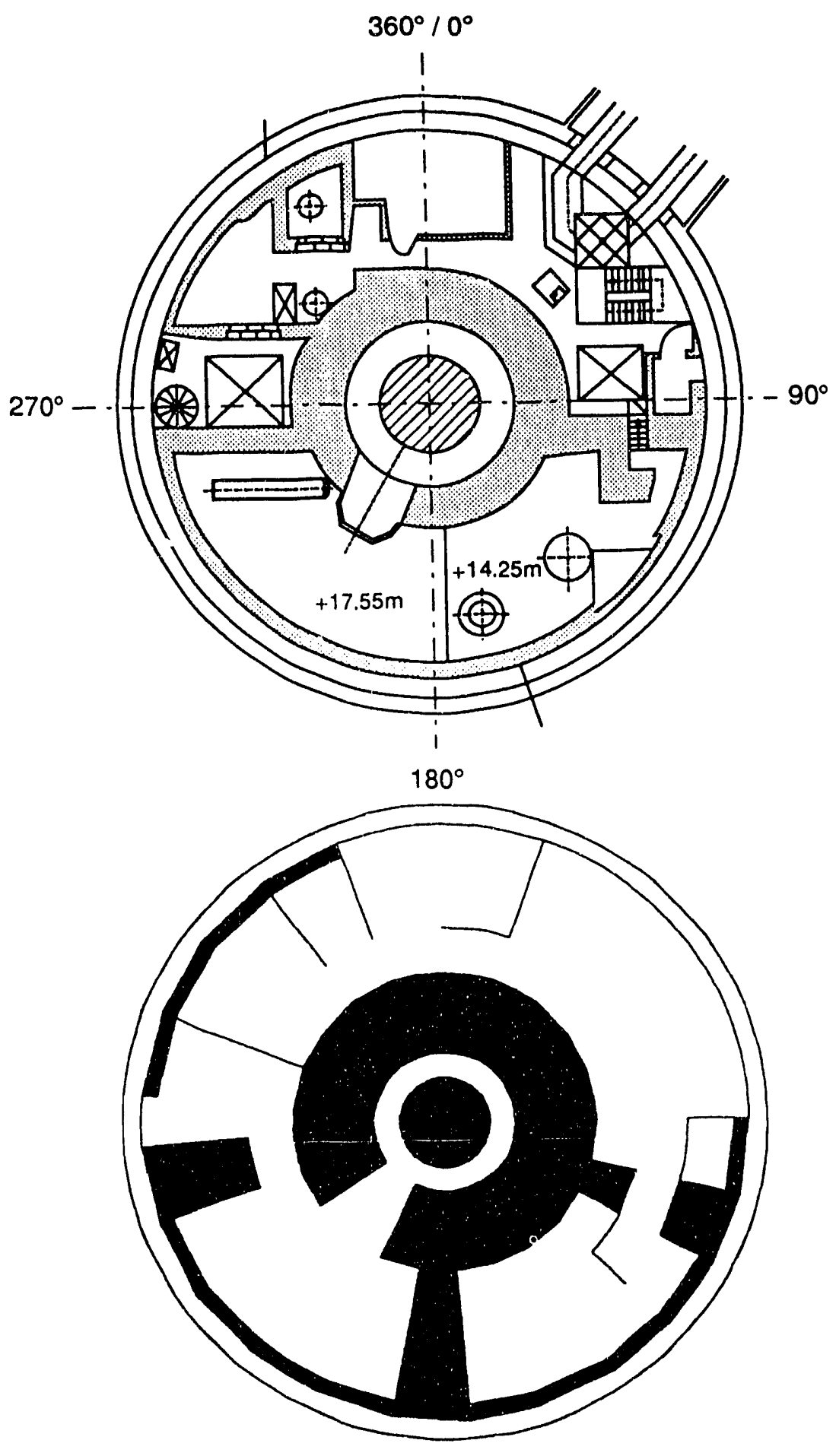

Fig. 30. Horizontal cross section of the HDR containment facility at $16 \mathrm{~m}$, with the physical drawing shown at the top and the HMS computational model at the bottom. 

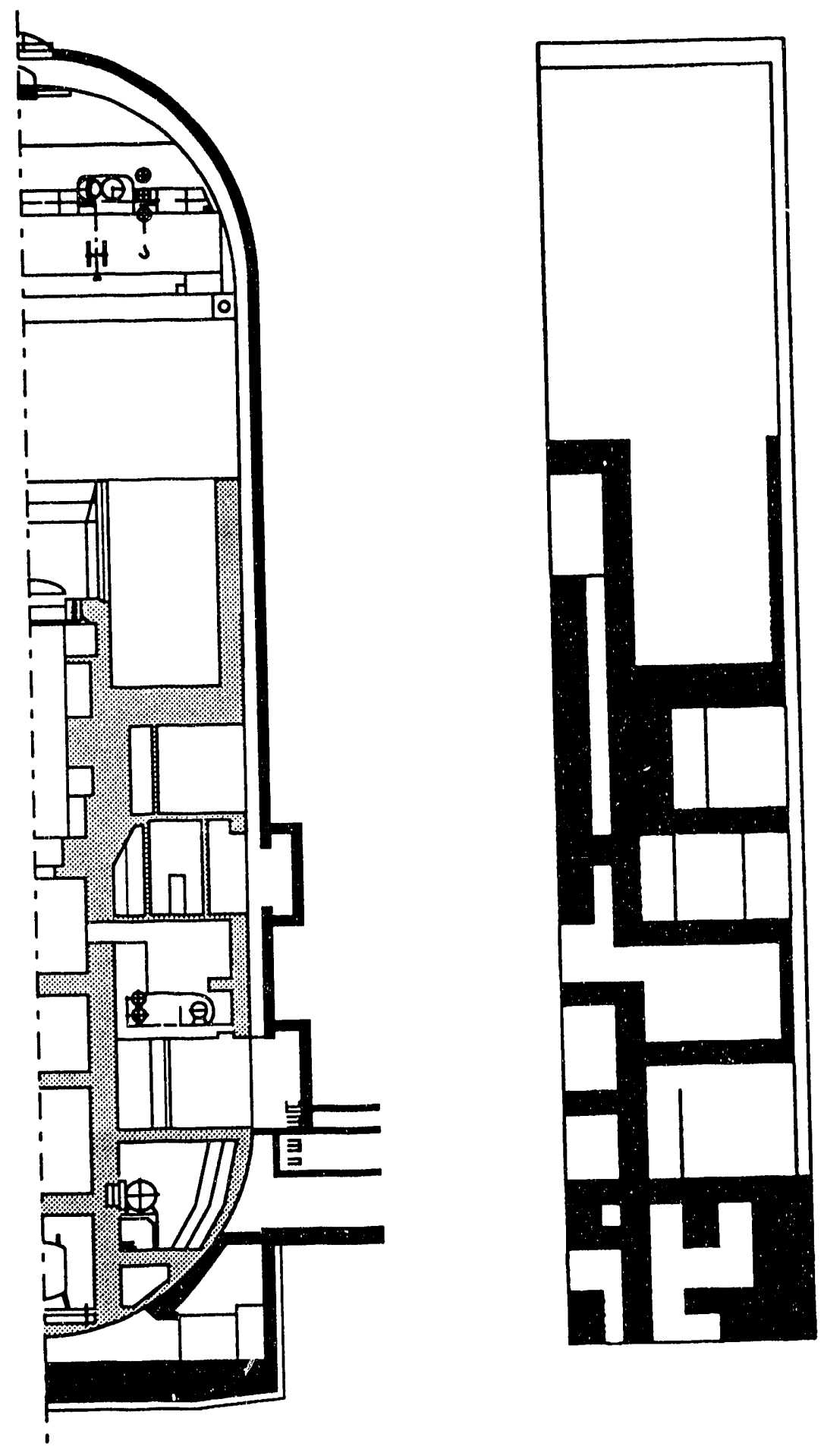

Fig. 31. HDR vertical cross section at $10^{\circ}$. The physical drawing is on the left and the HMS model is on the right. 


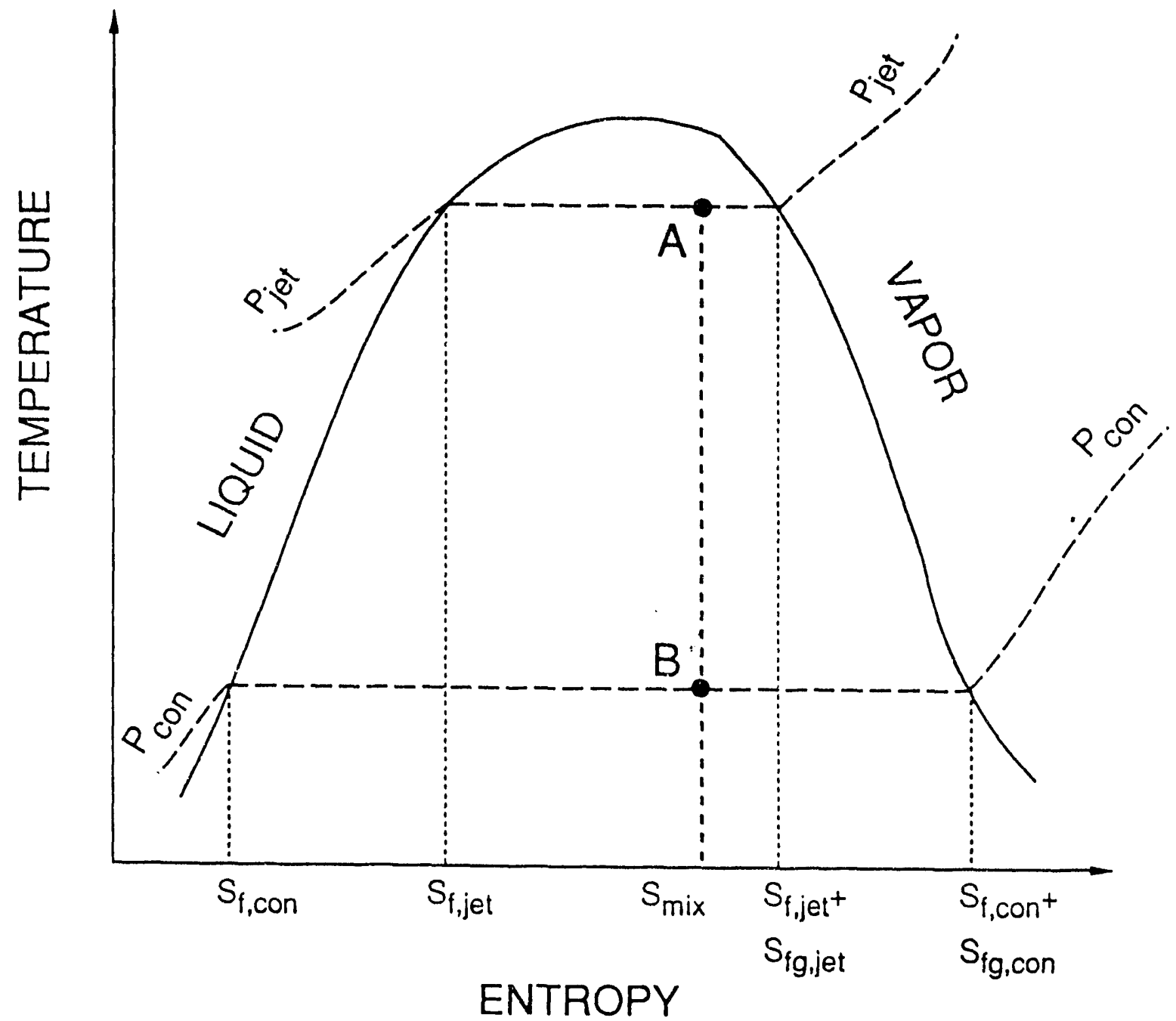

Fig. 32. Temperature-Entropy phase diagram (schematic) for water showing the isentropic expansion process from the blowdown jet pressure $\left(P_{\text {jet }}\right)$ and qualtity $\left(x_{j e t}\right)$ at Point $A$ to the containment pressure $\left(P_{\text {con }}\right)$ at Point $B$ following the constant-entropy line. The entropy of the two-phase mixture, $\mathrm{S}_{\mathrm{mix}}$ is assumed to be constant before and after the expansion. 

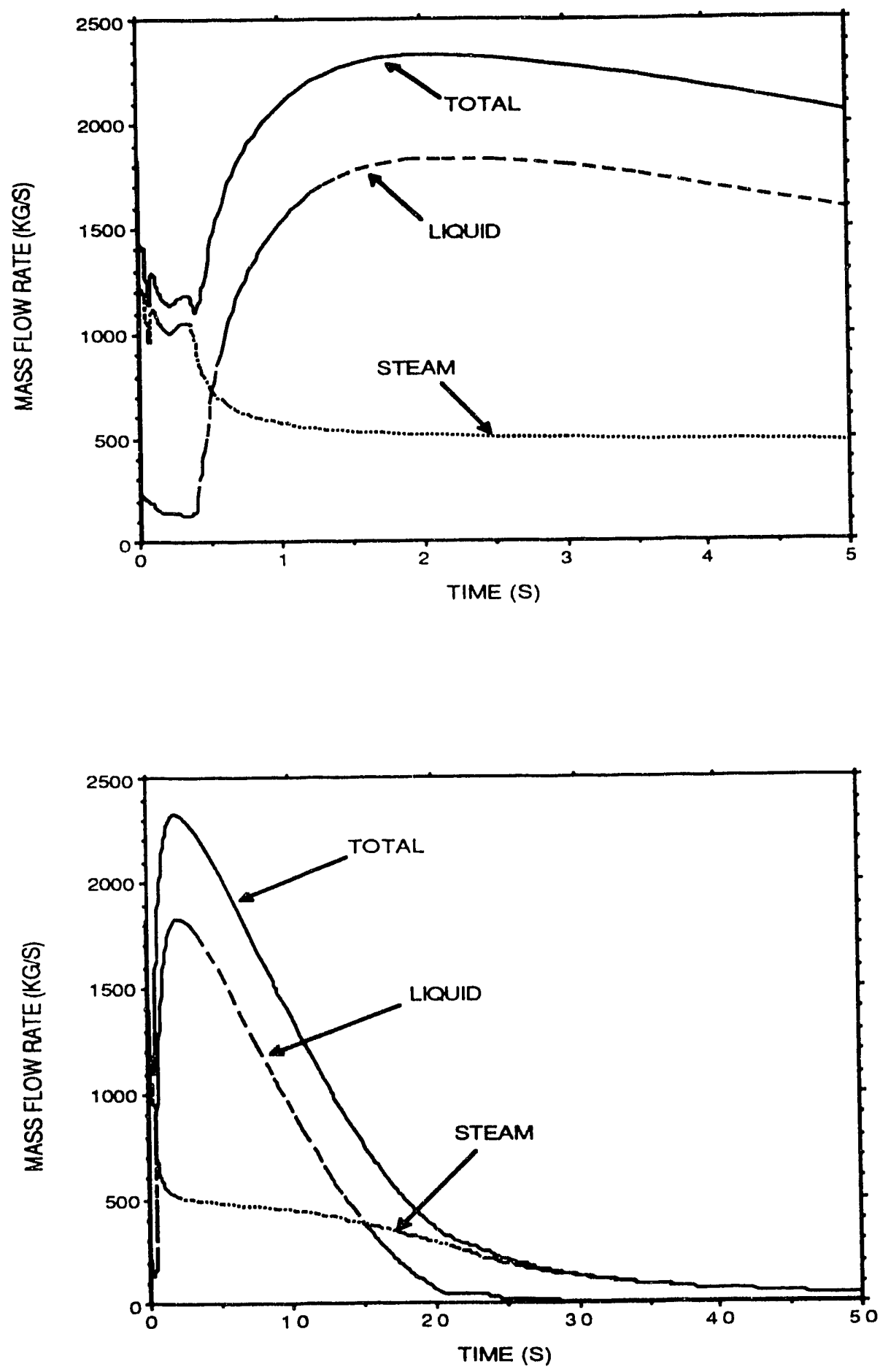

Fig. 33. Blowdown mass flow rates for two time intervals as given by the source data. Note that after about 26 seconds the blowdown is essentially all steam. 

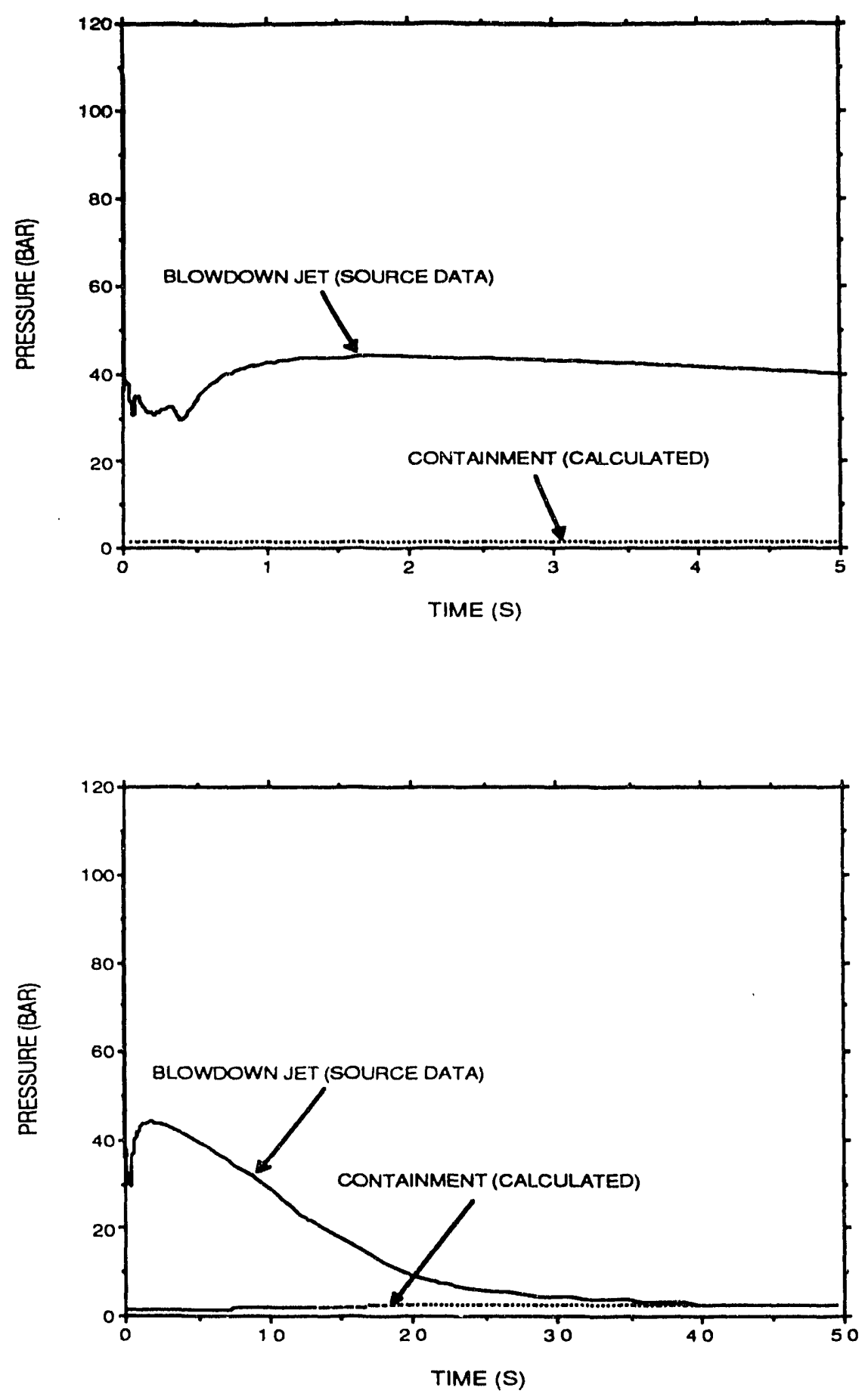

Fig. 34. Pressure for two time intervals in the blowdown jet (source data) and in the containment (calculated by HMS). The blowdown source is expanded isentropically from the jet pressure to the containment pressure to determine the correct amount of steam source into the containment. 

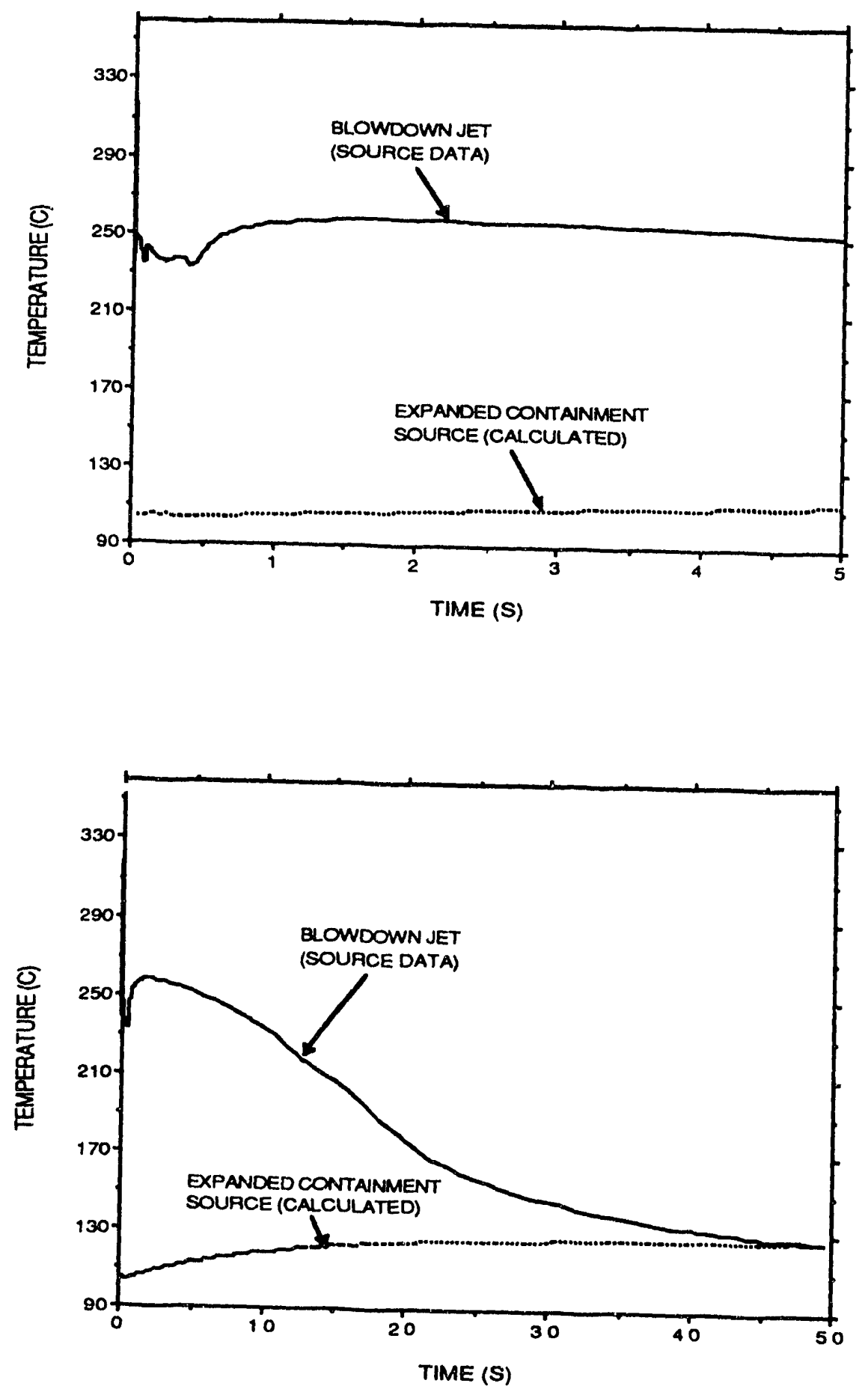

Fig. 35. Temperature for two time intervals in the blowdown jet (source data) and in the containment source (calculated) after the isentropic expansion process. 

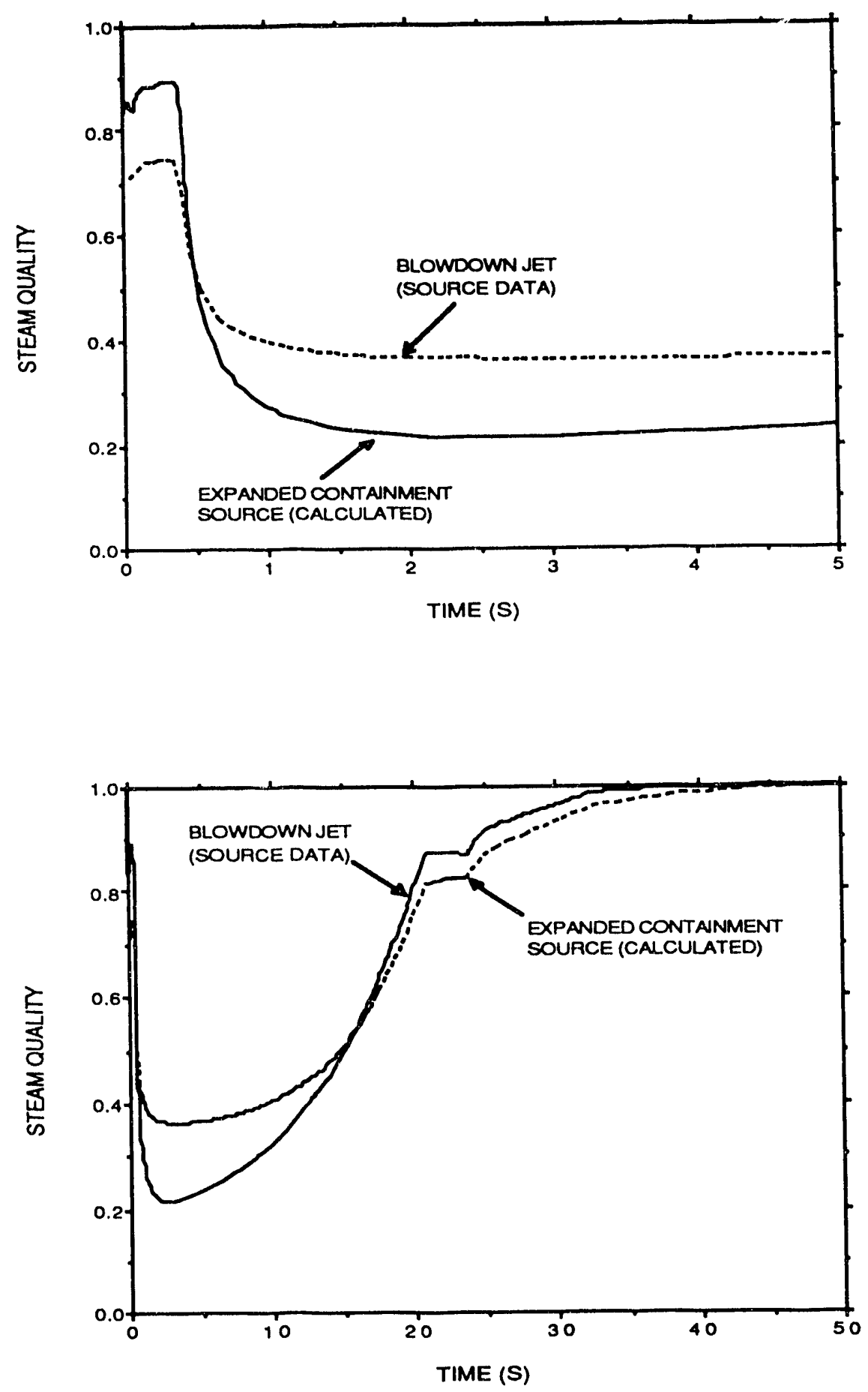

Fig. 36. Steam qualities in the blowdown jet and in the containment source after the isentropic expansion process for two time intervals. 

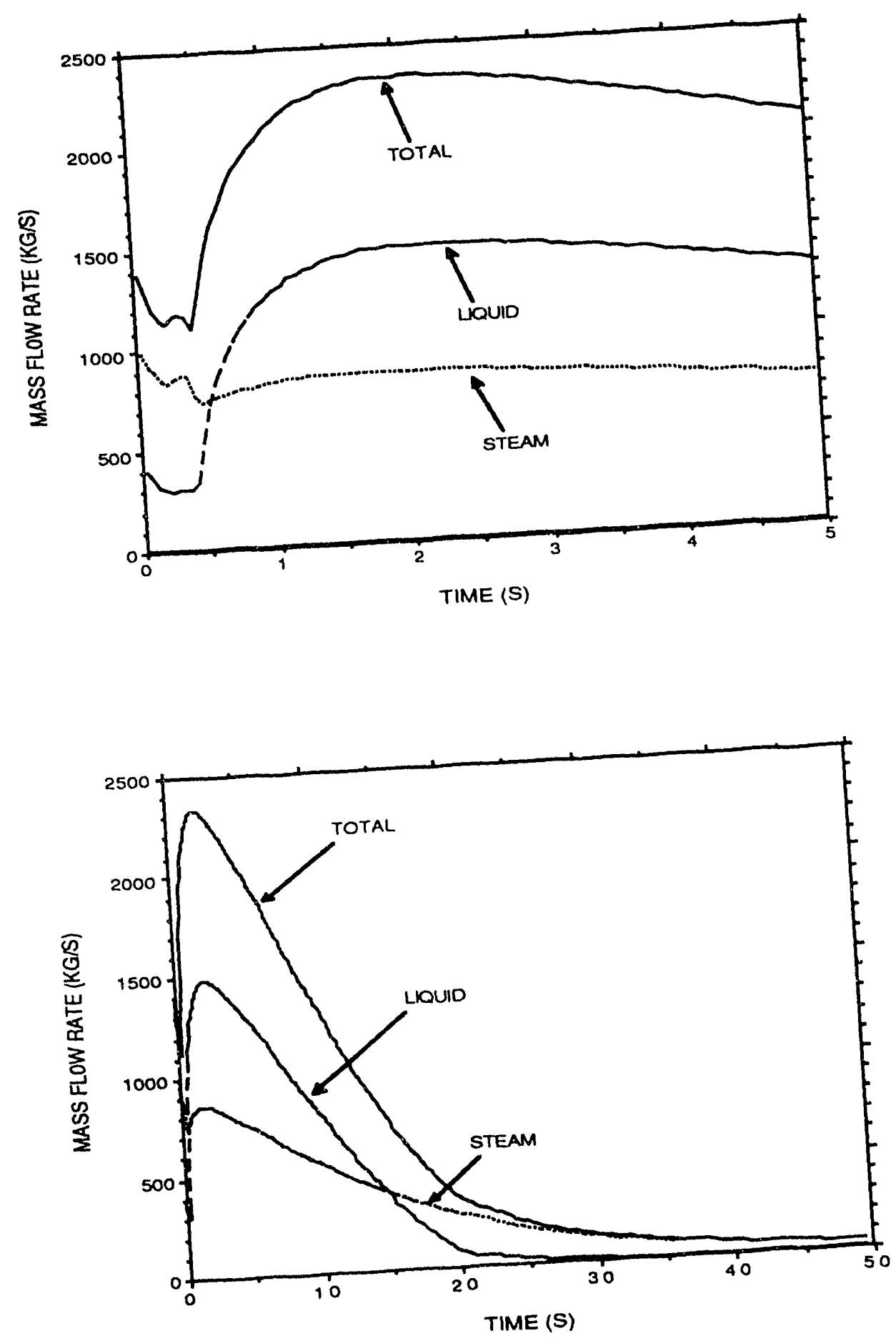

Fig. 37. Calculated steam and liquid source rates into the containment after the isentropic expansion process for two time intervals. 

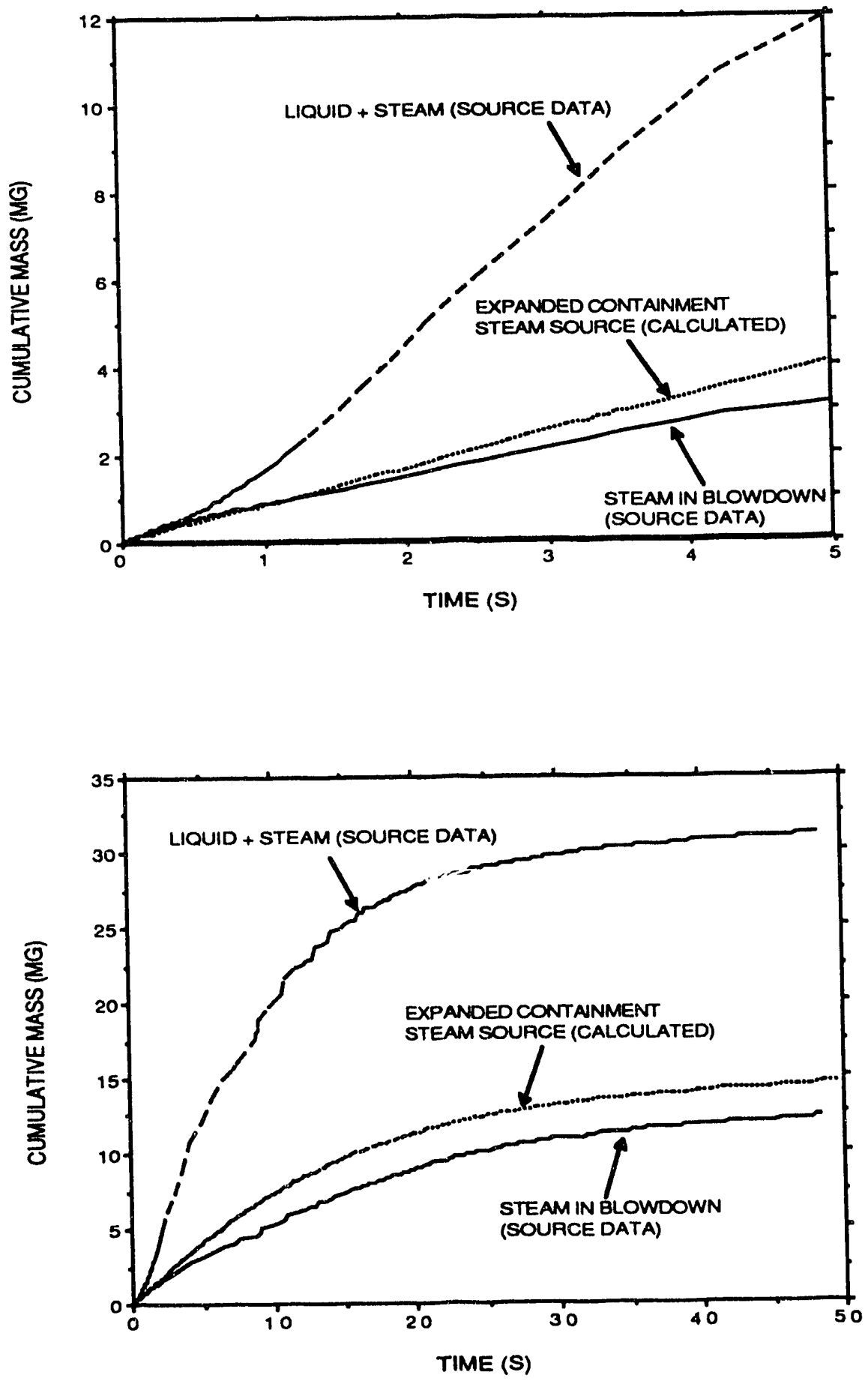

Fig. 38. Steam masses in original blowdown jet (source data) and in source to containment (calculated) for two time intervals. The cumulative mass of steam and water is plotted for reference. 

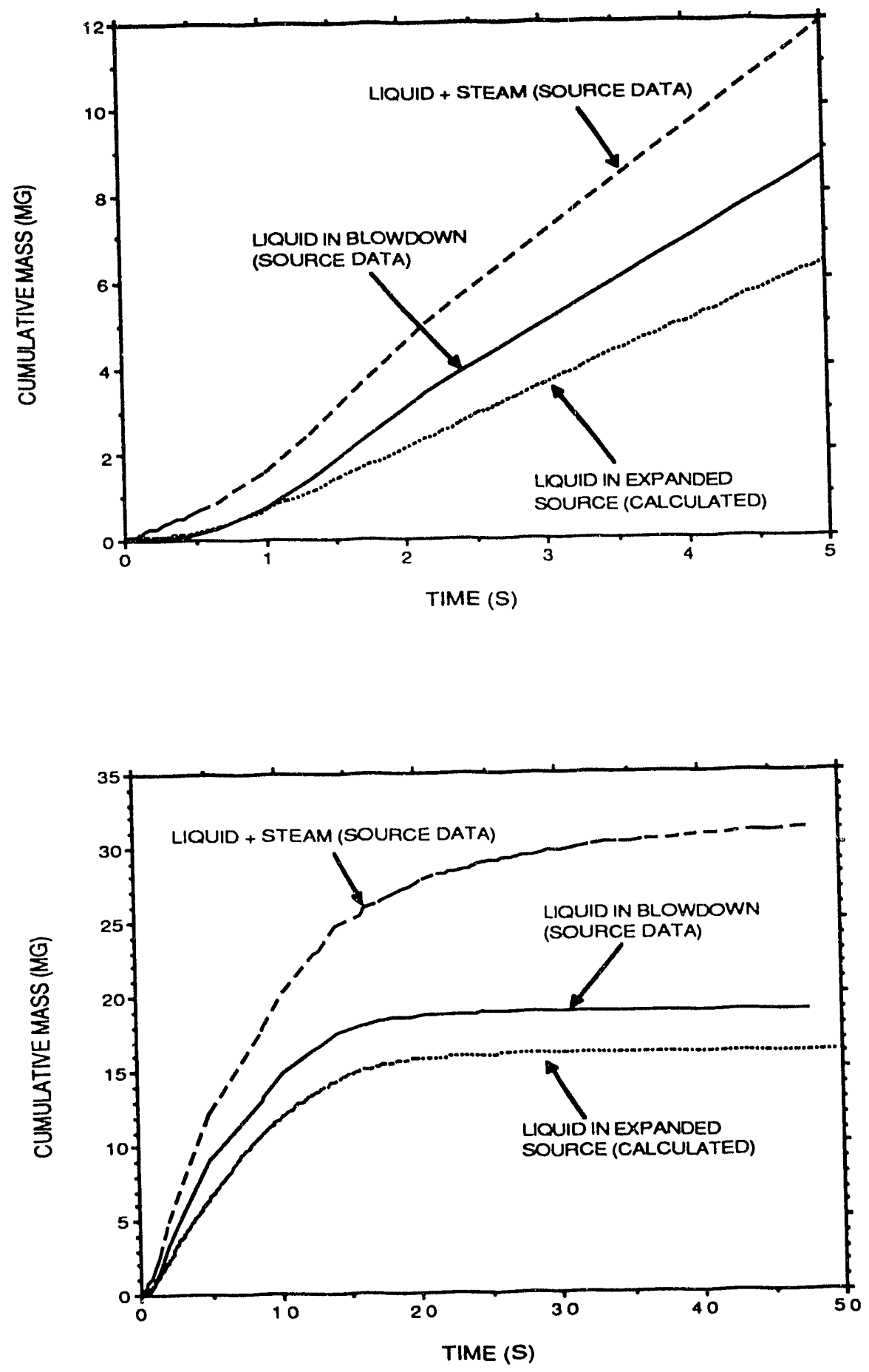

Fig. 39. Liquid masses in original blowdown jet (source data) and in expanded source to containment (calculated) for two time intervals. The cumulative mass of steam and water is also plotted for reference. 

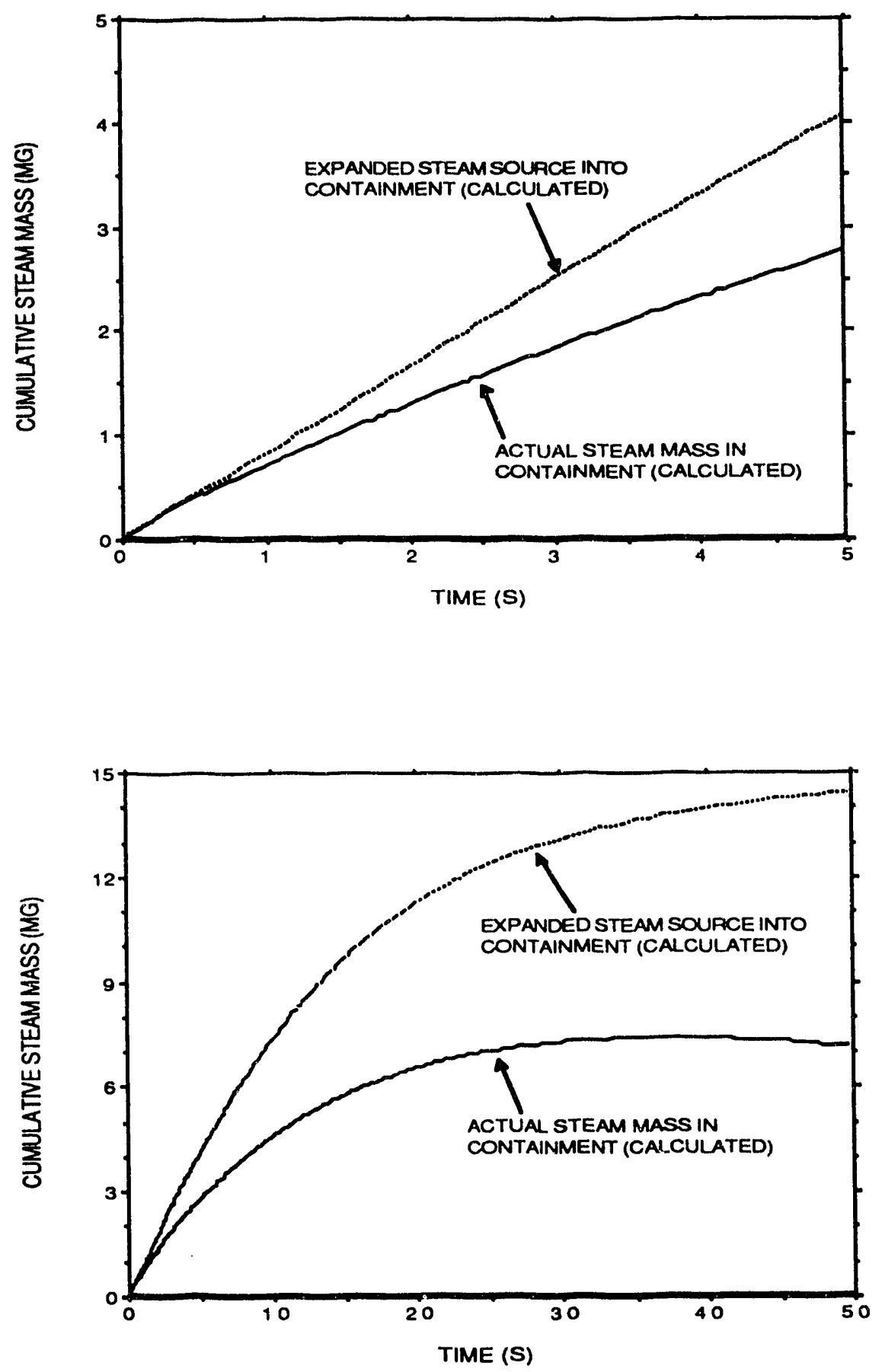

Fig. 40. Total amount of steam that has been injected into the containment (by the blowdown) compared with the actual amount of steam in the containment for two time intervals. The difference between the two curves indicates the amount condensed on surfaces in the containment during the transient. 

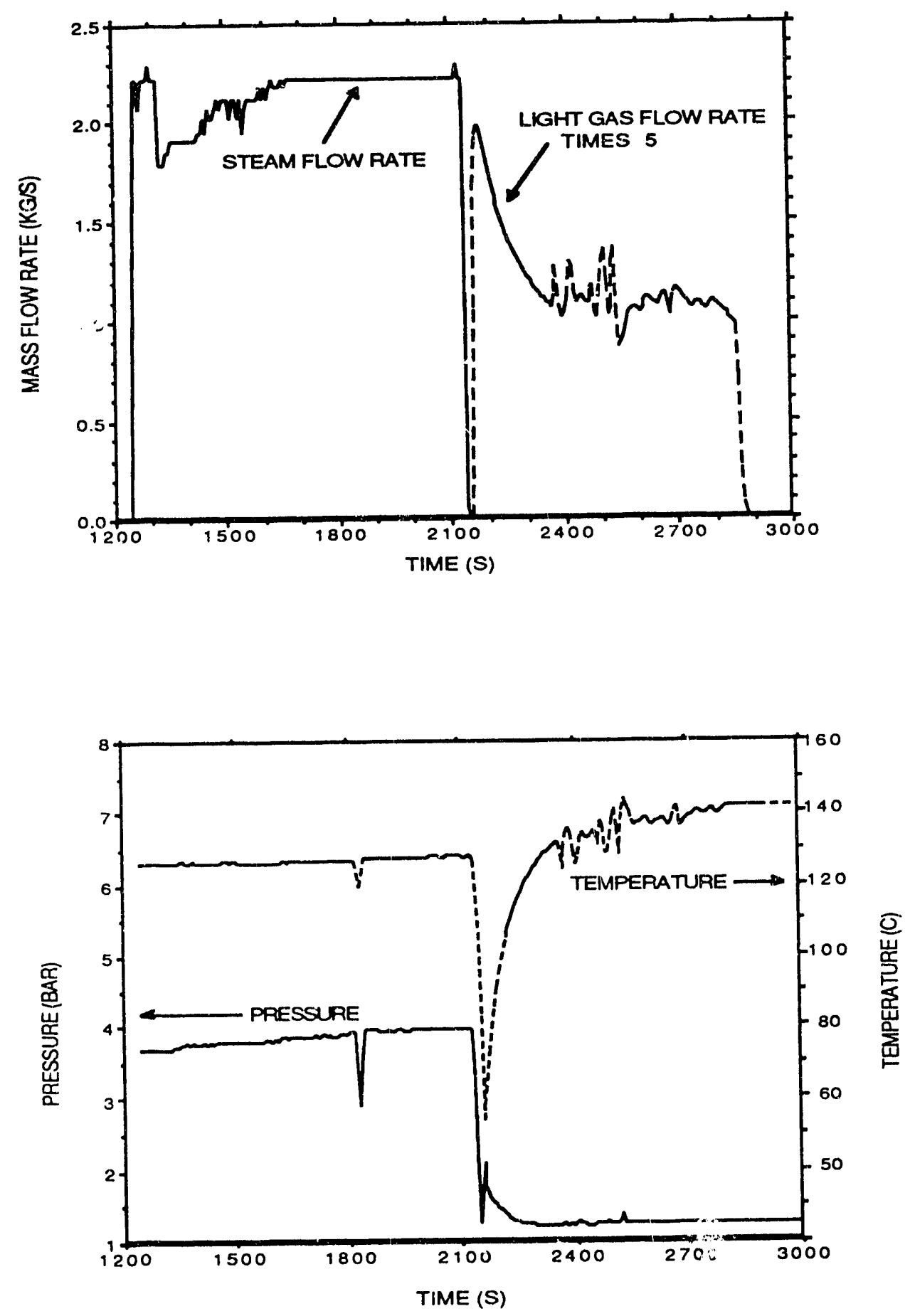

Fig. 41. Conditions $c^{\prime}$ superheated steam and light gas releases from $21 \mathrm{~min}$ to 50 min after beginning of the blowdown as given by the source data. The lop figure shows the mass release rates. The gas temperature and pressure are shown in the bottom figure 


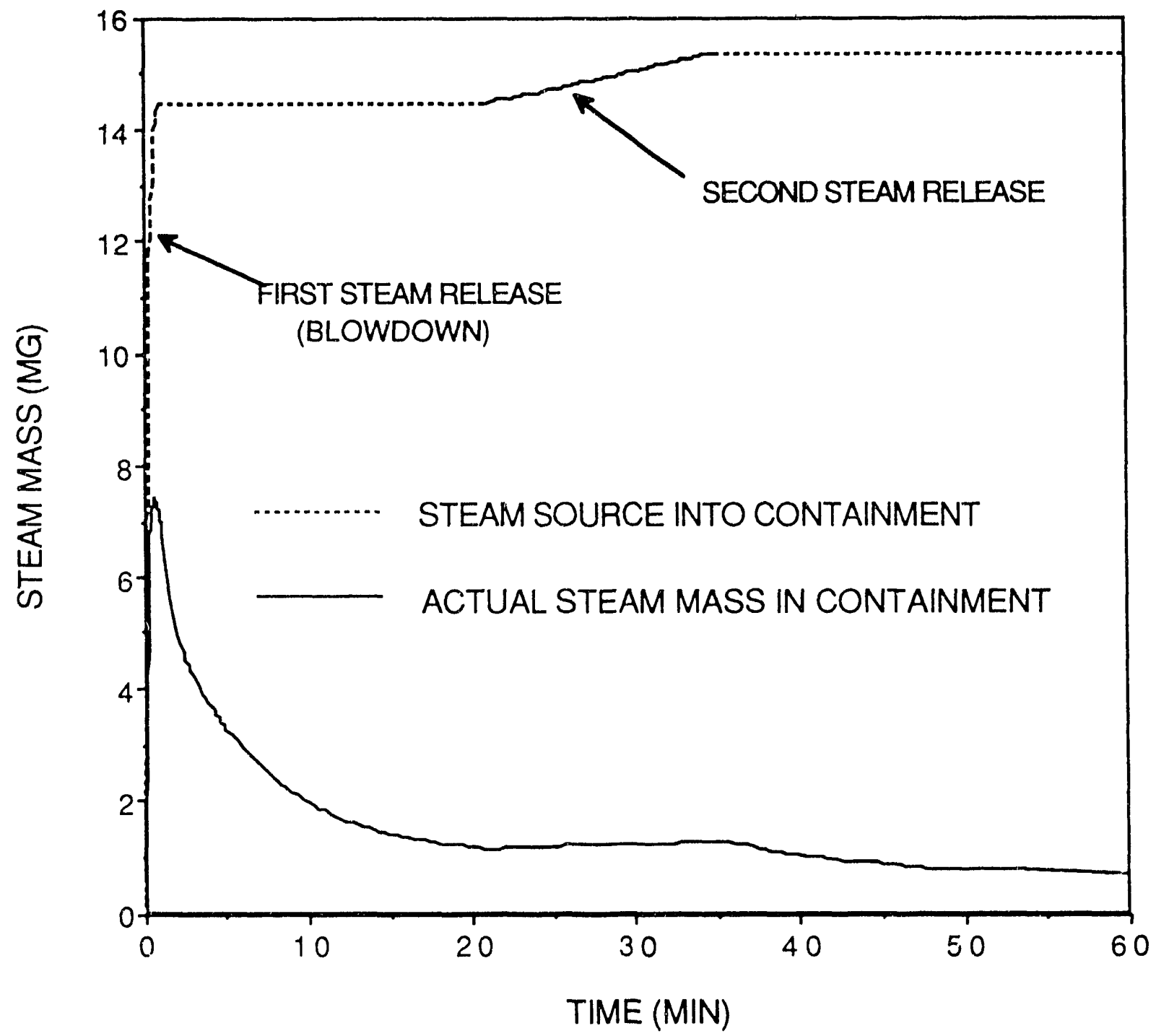

Fig. 42. Calculated cumulative steam source (after expansion from pipe to containment) and the actual steam mass (also calculated) in the containment during the first hour. The difference between the two curves indicates the amount of steam condensed on all internal surfaces. 

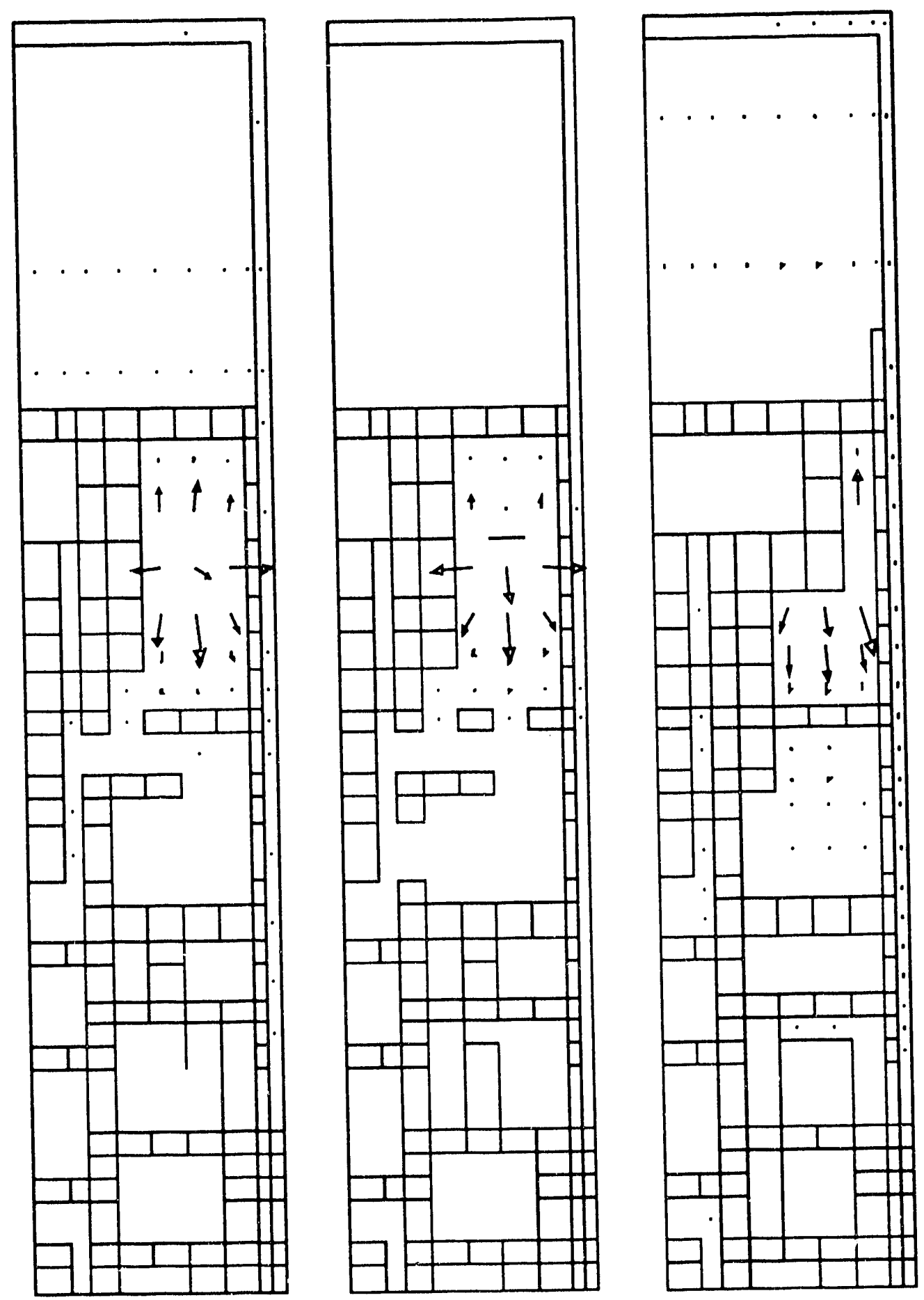

Fig. 43. Calculated velocity vector plots at three vertical $(R-Z)$ planes shortly $(7 \mathrm{~ms})$ after initiation of the large-break blowdown. The center plane cuts across the blowdown location while the left and right planes are at $15^{\circ}$ from it clockwise and counterclockwise, respectively. From left to right, the maximum velocity magnitudes (averaged at cell centers) for the plots are 8.2 $\mathrm{m} / \mathrm{s}, 34 \mathrm{~m} / \mathrm{s}$, and $1.8 \mathrm{~m} / \mathrm{s}$. 

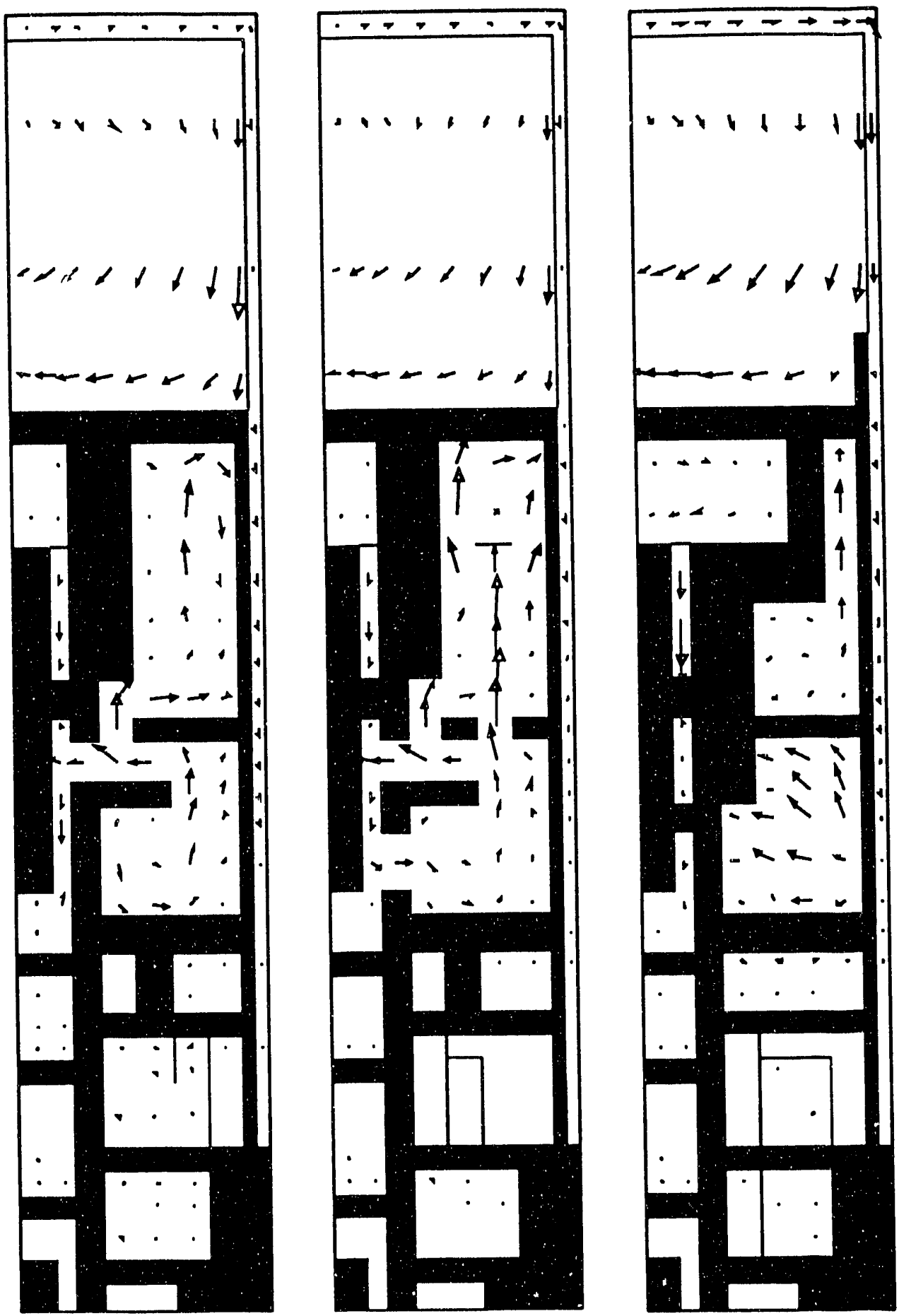

Fig. 44. Calculated velocity vector plots at $22 \mathrm{~min}$ at three $(\mathrm{R}-\mathrm{Z})$ vertical planes. The center plane cuts across the blowdown location while the left and right planes are at $15^{\circ}$ from it clockwise and counterclockwise, respectively. From left to right, the maximum velocity magnitudes (averaged at cell centers) for the plots are $0.65 \mathrm{~m} / \mathrm{s}, 0.86 \mathrm{~m} / \mathrm{s}$, and $0.45 \mathrm{~m} / \mathrm{s}$. 

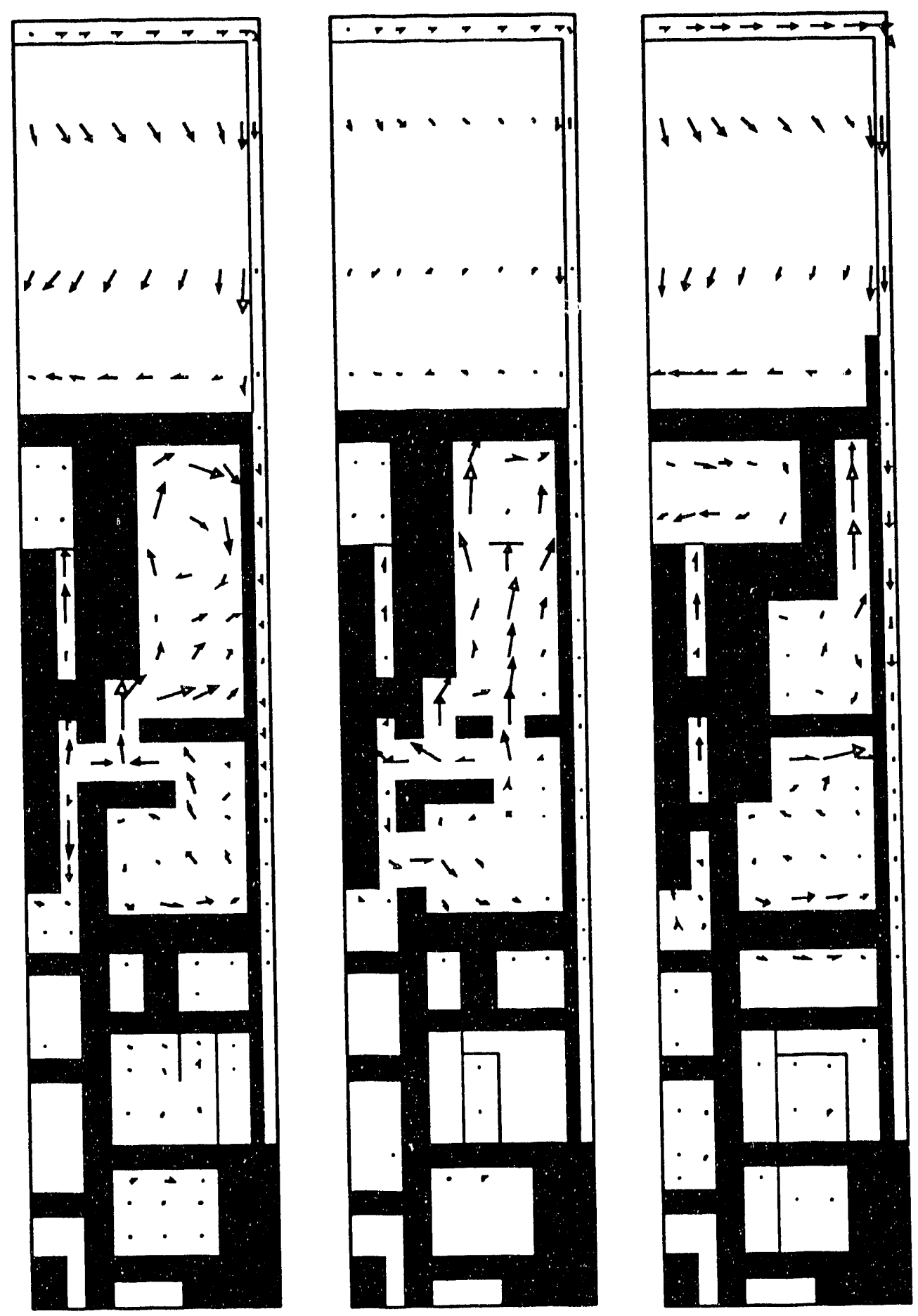

Fig. 45. Calculated velocity vector plots at $40 \mathrm{~min}$ at three vertical $(R-Z)$ planes. The center plane cuts across the blowdown location while the left and right planes are at $15^{\circ}$ from it clockwise and counterclockwise, respectively. From left to right, the maximum velocity magnitudes (averaged at cell centers) for the plots are $0.45 \mathrm{~m} / \mathrm{s}, 0.91 \mathrm{~m} / \mathrm{s}$, and $0.27 \mathrm{~m} / \mathrm{s}$. 


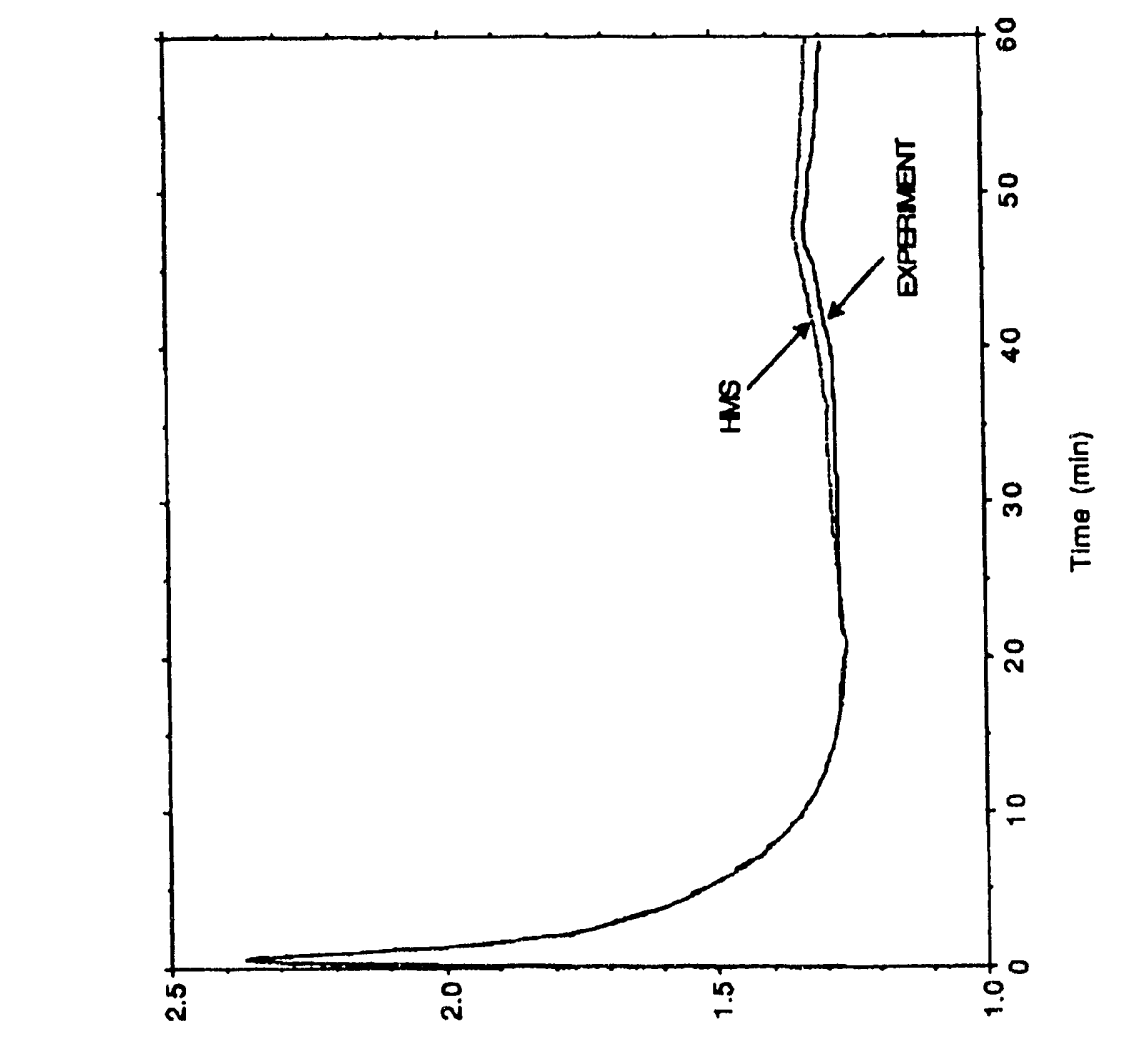

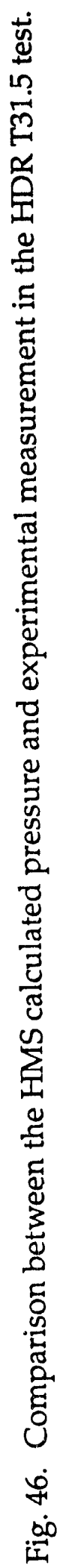

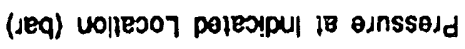

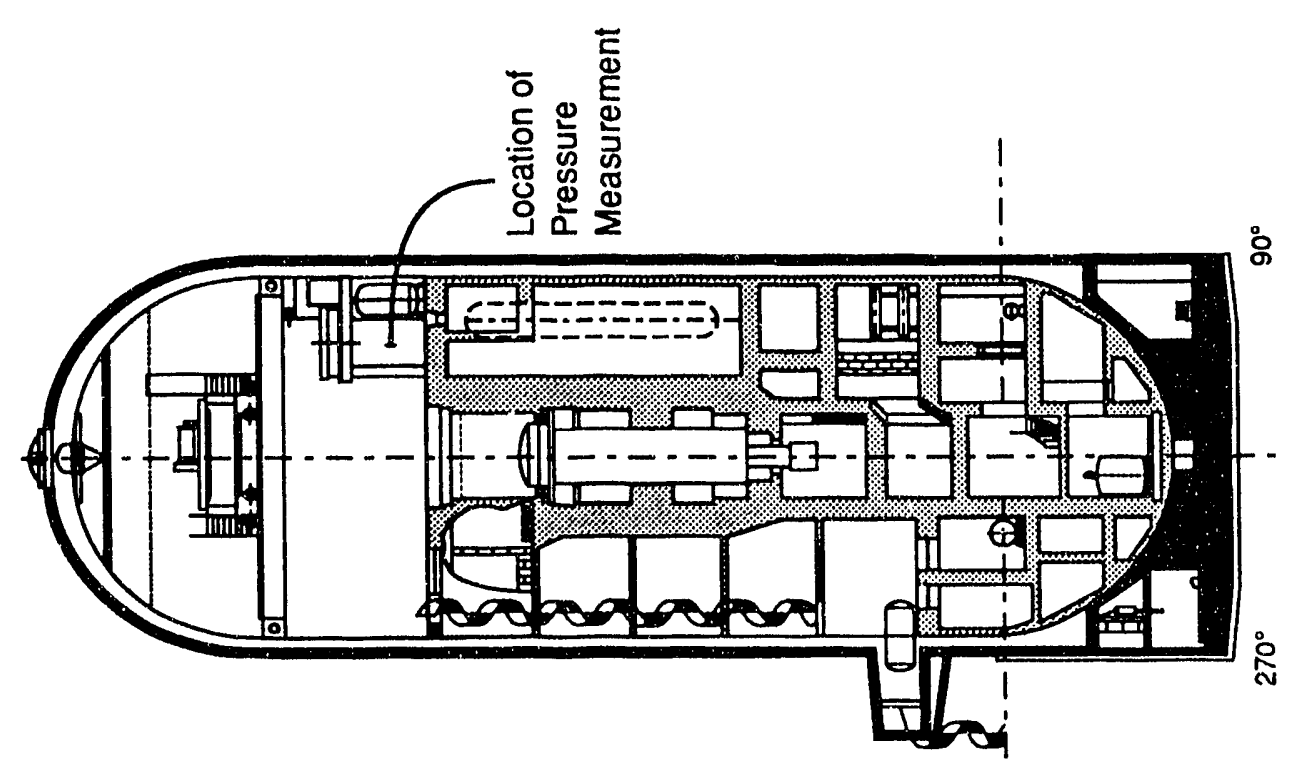



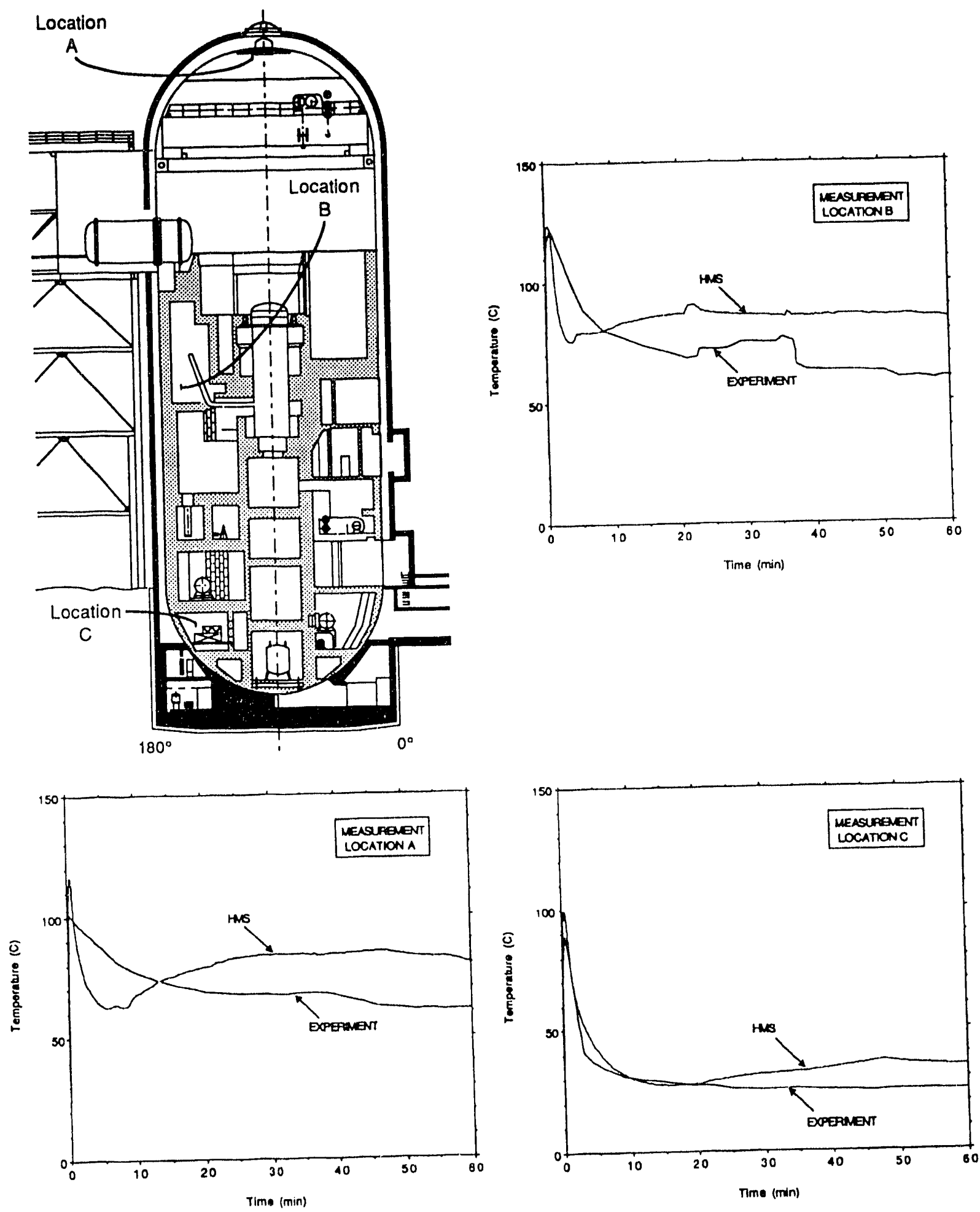

Fig. 47. Comparison between the HMS calculated temperature and experimental measurement at three locations in the HDR T31.5 test. 

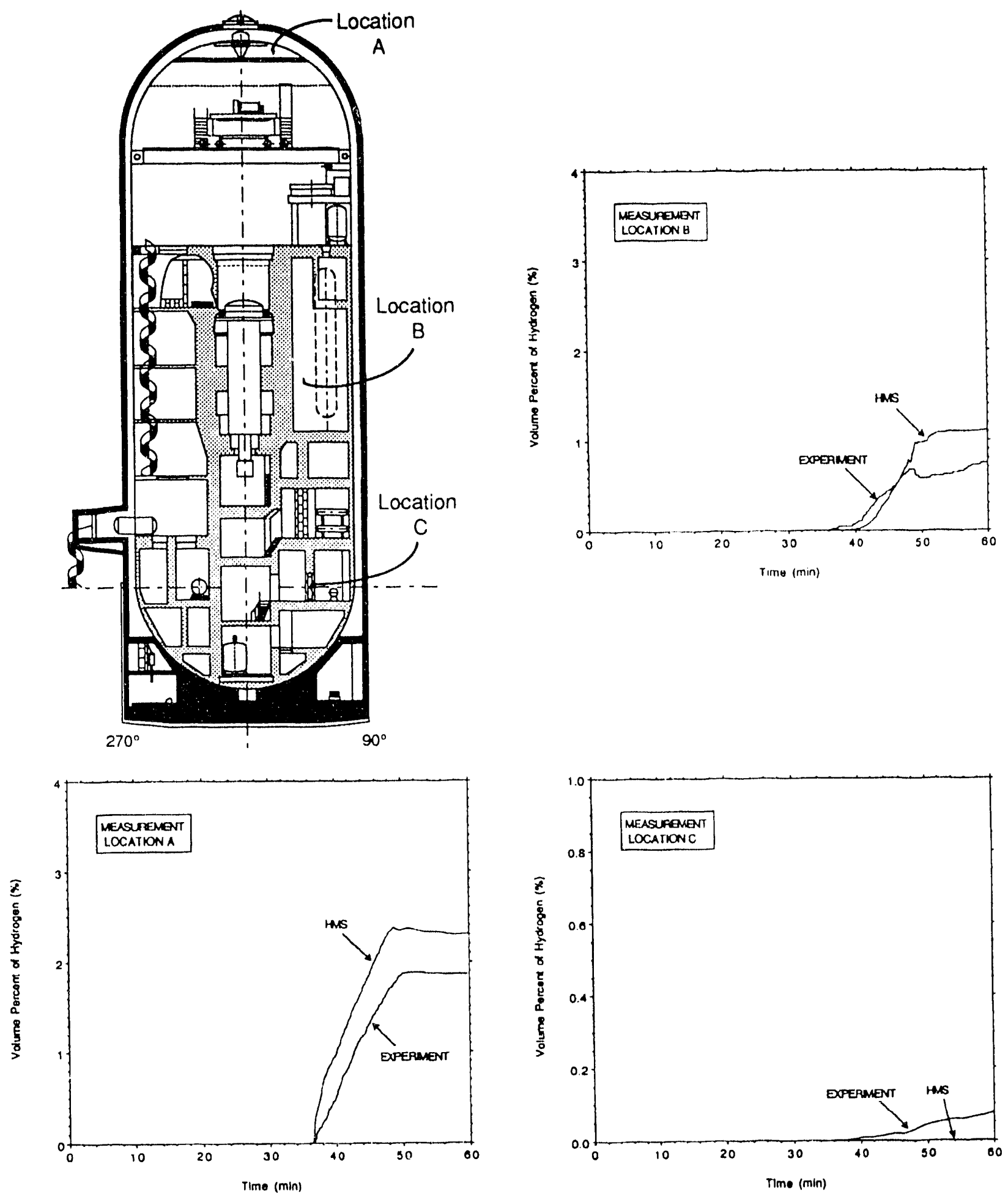

Fig. 48. Comparison between the HMS-calculated hydrogen concentration and experimental measurement at three locations in the HDR T31.5 test. 

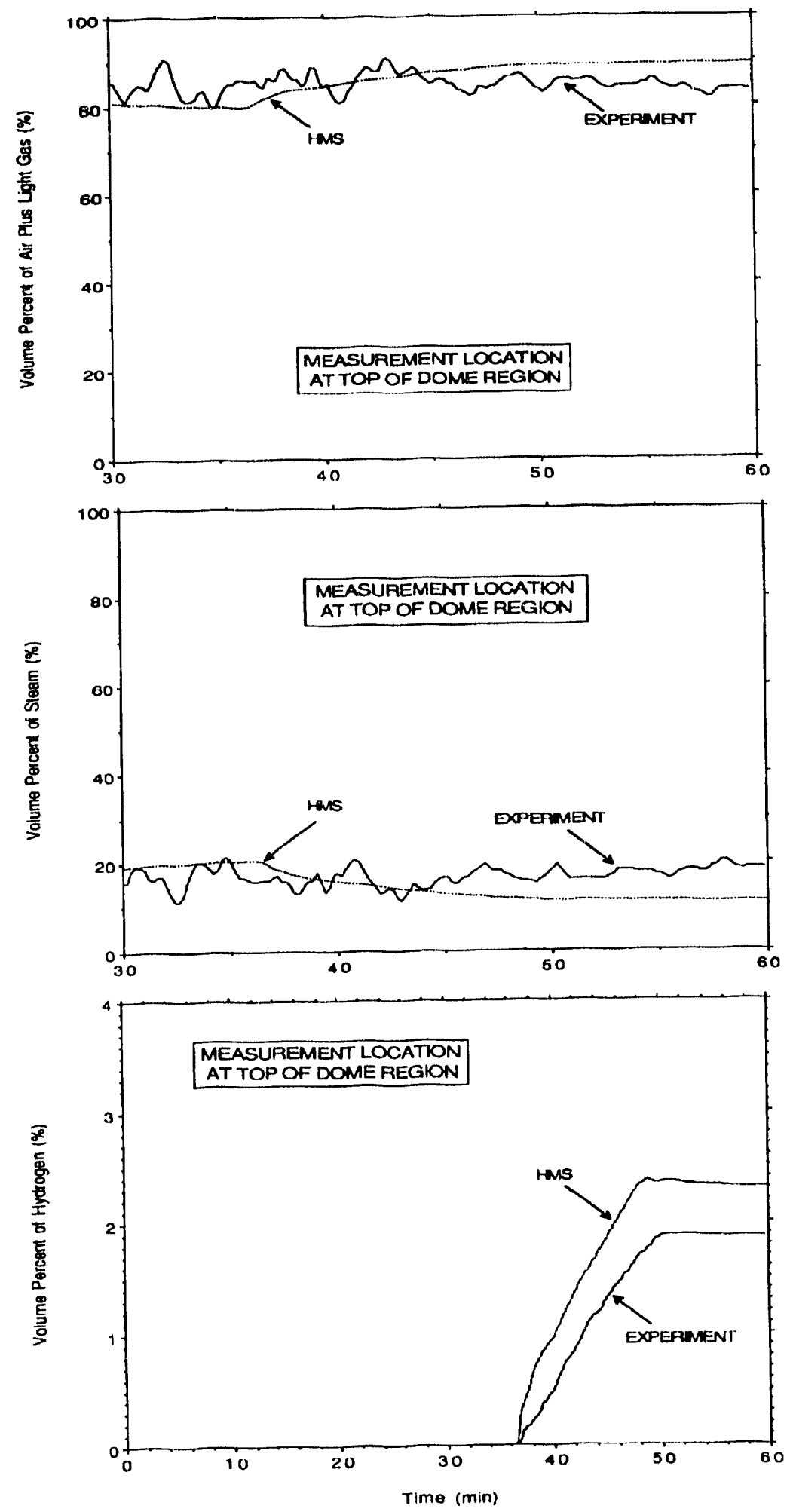

Fig. 49. Measured and HMS-calculated concentrations of air, light gas (15\% hydrogen and $85 \%$ helium by volume), steam, and hydrogen at the top of the dome (LOCATION A in Fig. 48) in the HDR T31.5 test. 

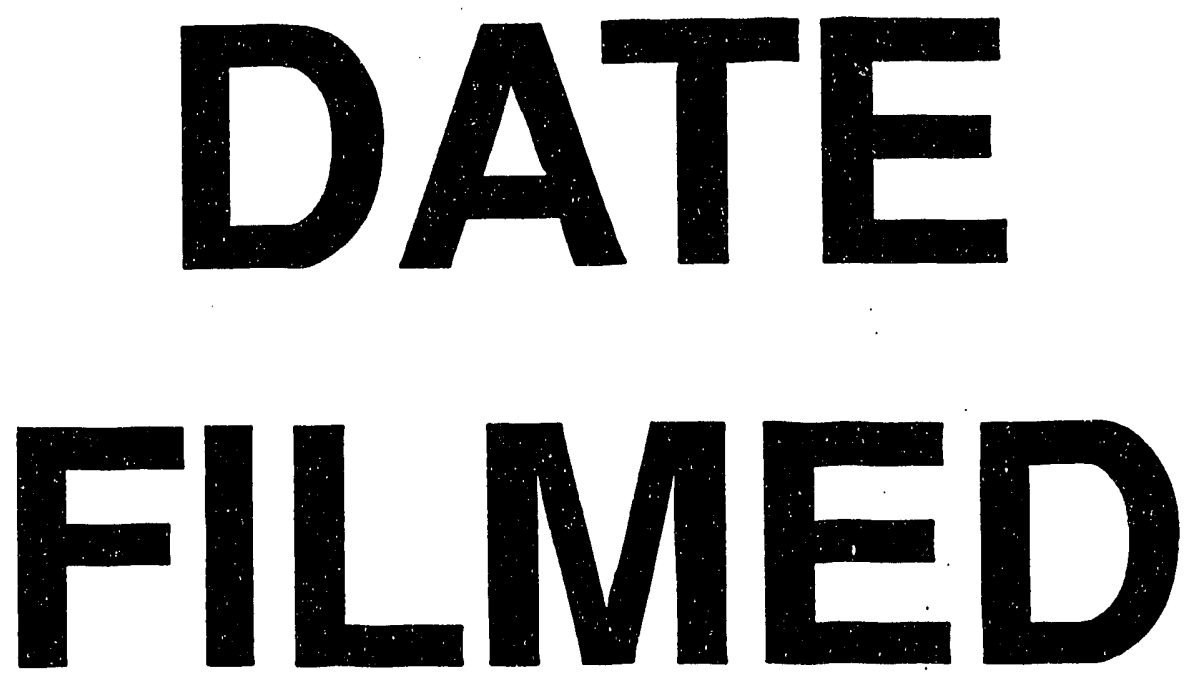

$9 / 23 / 93$
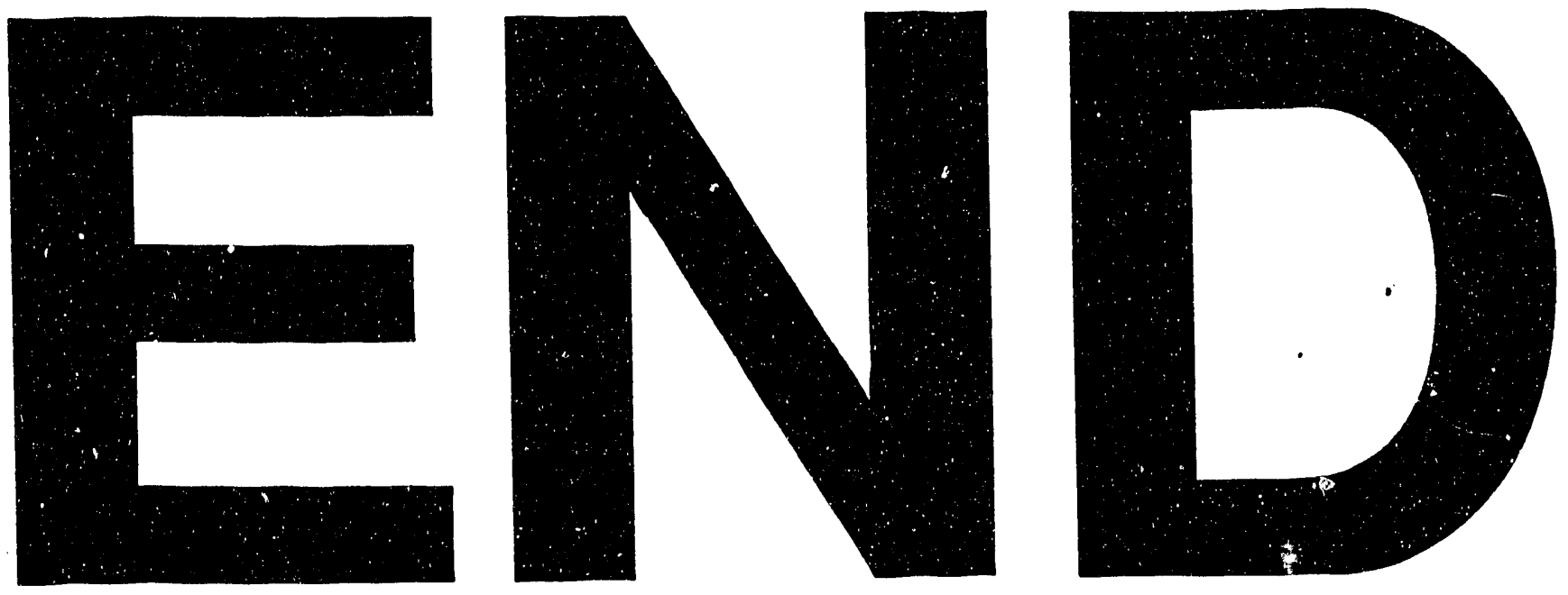
